TRANSACTIONS OF THE

AMERICAN MATHEMATICAL SOCIETY

Volume 356, Number 7, Pages 2569-2648

S 0002-9947(03)03479-2

Article electronically published on October 29, 2003

\title{
THOMASON'S THEOREM FOR VARIETIES OVER ALGEBRAICALLY CLOSED FIELDS
}

\author{
MARK E. WALKER
}

\begin{abstract}
We present a novel proof of Thomason's theorem relating Bott inverted algebraic $K$-theory with finite coefficients and étale cohomology for smooth varieties over algebraically closed ground fields. Our proof involves first introducing a new theory, which we term algebraic $K$-homology, and proving it satisfies étale descent (with finite coefficients) on the category of normal, Cohen-Macaulay varieties. Then, we prove algebraic $K$-homology and algebraic $K$-theory (each taken with finite coefficients) coincide on smooth varieties upon inverting the Bott element.
\end{abstract}

\section{INTRODUCTION}

In this paper we present a novel proof of Thomason's celebrated theorem [27. 4.1] comparing the Bott inverted algebraic $K$-groups with finite coefficients and étale cohomology. Our result applies to smooth quasi-projective varieties defined over an algebraically closed ground field, and is thus not as general as Thomason's original theorem, which imposes no restriction on the ground field. However, the proof given in this paper is arguably more elementary than that in Thomason's, and in particular we do not rely on any of the complicated constructions in the category of spectra employed by Thomason.

Recently, a new proof of Thomason's theorem has been given by Levine [17, 13.1] building on the recently established motivic Atiyah-Hirzebruch spectral sequence developed in [3, [8, and [17]. Our proof also does not use in any fashion this spectral sequence.

Throughout this paper, we frequently assume the following condition on the integer $n$ used for the coefficient group $\mathbb{Z} / n$ :

(Condition 2-3)

If $3 \mid n$, then $9 \mid n$, and if $2 \mid n$, then $16 \mid n$.

The main result of this paper is

Theorem 1.1 (see Theorem 10.2). Let $X$ be a smooth, quasi-projective variety defined over an algebraically closed ground field $k$. Assume $n \geq 1$ is an integer relatively prime to the exponential characteristic of $k$ and such that $n$ satisfies (Condition 2-3). Then there is a convergent spectral sequence (concentrated in the portion of the pq-plane satisfying $p \geq q \geq p-2 \operatorname{dim}(X))$ having the form

$$
E_{2}^{p, q}=\mathrm{H}_{e ́ t}^{p-q}(X, \mathbb{Z} / n) \Longrightarrow K_{-p-q}(X ; \mathbb{Z} / n)[1 / \beta],
$$

Received by the editors August 24, 2002.

2000 Mathematics Subject Classification. Primary 19E15, 19E20, 14F20.

Key words and phrases. Algebraic K-theory, étale cohomology, Thomason's theorem.

(C)2003 American Mathematical Society 
where $K_{m}(X ; \mathbb{Z} / n)[1 / \beta]$ denotes the $m$-th graded piece of the ring obtained by inverting the action of the Bott element $\beta \in K_{2}(\operatorname{Spec} k ; \mathbb{Z} / n)$ on $K_{*}(X ; \mathbb{Z} / n)$.

The techniques used to prove Theorem 1.1 also allow for a comparison result relating $K$-theory and Bott-inverted $K$-theory (with finite coefficients). The precise statement is

Theorem 1.2 (see Theorem[10.4). Let $X, d, k$, and $n$ be as in Theorem 1.1. Then the map

$$
K_{*}(X ; \mathbb{Z} / n) \rightarrow K_{*}(X ; \mathbb{Z} / n)[1 / \beta]
$$

is a split surjection in degrees $* \geq 2 d$, and in all degrees the kernel of this map is killed by $\beta^{d}$.

Theorem 1.1 itself is not new, but the techniques we use to prove it are novel and perhaps of independent interest. In particular, and as in the author's previous paper [36] (in which the main result of the current paper was proven in the special case where $k=\mathbb{C}$ ), our proof uses properties of a new theory which we term "algebraic $K$-homology", $K_{*}^{\text {hom }}$.

Loosely speaking, the algebraic $K$-homology of a variety $Y$ is defined by considering parameterized families of coherent sheaves of finite length on $Y$. Algebraic $K$-homology is analogous to connective topological $K$-homology, but should not be confused with $G$-theory (also known as $K^{\prime}$-theory, defined from the category of coherent sheaves). For one thing, algebraic $K$-homology is a true homology theory, being covariantly functorial for all morphisms of varieties, whereas $G$-theory enjoys the functoriality of a "Borel-Moore" type theory. However, it is not even the case that $G$-theory represents the Borel-Moore theory associated to $K$-homology, as seen by the calculation

$$
K_{0}^{\operatorname{hom}}(Y) \cong C H_{0}(Y)
$$

given in Proposition 3.7 below. Thus, $K$-homology and $G$-theory do not coincide even for projective varieties (except for the trivial case of a point).

The distinction between algebraic $K$-homology and $G$-theory is explained, at least heuristically, by observing that $K$-homology is a truncated form of $G$-theory, involving only that portion of $G$-theory having weight at least equal to the dimension of a given variety (i.e., that portion of $G$-theory arising from coherent sheaves with zero-dimensional support). If such a truncation strikes the reader as odd, we note that the construction of $K$-homology is highly analogous to a certain construction of connective topological $K$-theory - the author's previous paper 36 makes this connection very precise. Moreover, since the Bott element $\beta$ has weight one, such a truncation should not affect the theory upon inversion of $\beta$. The utility of truncating $G$-theory in this manner is hinted at by a theorem of Suslin 26] (see also Theorem (7.3), which states that for a smooth variety $X$ defined over an algebraically closed ground field, the motivic cohomology with finite coefficients of weight at least $\operatorname{dim}(X)$ of $X$ coincides with the étale cohomology of $X$. Since $K$ theory is related to motivic cohomology (in a manner which is made precise by the motivic spectral sequence, but which we do not use in this paper), it is reasonable to expect algebraic $K$-homology to enjoy étale descent. In fact, this theorem of Suslin not only provides motivation for the results of this paper but is indeed utilized in the proofs of Theorems 7.6 and 7.8 . 
To prove our main theorem for all quasi-projective varieties, we also utilize the "Borel-Moore theory" associated to $K_{*}^{\text {hom }}$, written $K_{*}^{\mathrm{BM}}$, which coincides with $K_{*}^{\text {hom }}$ on projective varieties and fits into the expected localization long exact sequence (see Corollary 5.6).

Using broad strokes, our proof of Theorem 1.1 is given by establishing the following ingredients. Let $k, X$, and $n$ satisfy the hypotheses of Theorem 1.1 .

(A) The graded abelian group $K_{*}^{\mathrm{BM}}(X ; \mathbb{Z} / n)$ is a graded module (under cap product $\cap$ ) over the graded ring (under cup product $\cup$ ) $K_{*}(X ; \mathbb{Z} / n$ ) (see Section 3).

(B) There exists a "fundamental class" $[X]$ belonging to $K_{2 \cdot \operatorname{dim}(X)}^{\mathrm{BM}}(X ; \mathbb{Z} / n)$ and a "duality map" $\gamma_{X}: K_{*}^{\mathrm{BM}}(X ; \mathbb{Z} / n) \rightarrow K_{*}(X ; \mathbb{Z} / n)$ such that the compositions

$$
K_{*}^{\mathrm{BM}}(X ; \mathbb{Z} / n) \stackrel{\gamma_{X}}{\longrightarrow} K_{*}(X ; \mathbb{Z} / n) \stackrel{-\cap[X]}{\longrightarrow} K_{*+2 \cdot \operatorname{dim}(X)}^{\mathrm{BM}}(X ; \mathbb{Z} / n)
$$

and

$$
K_{*}(X ; \mathbb{Z} / n) \stackrel{-\cap[X]}{\longrightarrow} K_{*+2 \cdot \operatorname{dim}(X)}^{\mathrm{BM}}(X ; \mathbb{Z} / n) \stackrel{\gamma_{X}}{\longrightarrow} K_{*+2 \cdot \operatorname{dim}(X)}(X ; \mathbb{Z} / n)
$$

are given by multiplication by $\beta^{2 \cdot \operatorname{dim}(X)}$ under cap and cup product, respectively (see Theorem 8.13). In particular, $K_{*}^{\mathrm{BM}}(X ; \mathbb{Z} / n)[1 / \beta]$ is a graded free module of rank one over the graded $\operatorname{ring} K_{*}(X ; \mathbb{Z} / n)[1 / \beta]$.

(C) Borel-Moore $K$-homology with finite coefficients, $K_{*}^{\mathrm{BM}}(-; \mathbb{Z} / n)$, satisfies étale descent (without having to invert the Bott element) on the category of normal, Cohen-Macaulay varieties over $k$ (see Theorems 7.6 and 7.8).

The main theorem follows more or less directly from these three results. The proofs of results (A) and (B) are rather formal in nature, but do require a fair amount of technical machinery. The proof of (C) ultimately amounts to an application of Suslin "rigidity" (although, it too requires a significant amount of technical machinery).

We now briefly outline the contents of each of the sections of this paper. Section 2 is devoted merely to recording formal definitions of the rather large number of exact categories and associated $K$-theory spaces used throughout the remainder of the paper. The reader might treat this section as a sort of glossary, not to be read in a linear fashion but rather referred to as the need arises. In Section 3 we introduce the main objects of study: algebraic $K$-homology $\mathcal{K}^{\text {hom }}$, its associated Borel-Moore theory $\mathcal{K}^{B M}$, and two bivariant theories $\mathcal{K}_{\text {geom }}(-\rightarrow-)$ and $\mathcal{K}_{\text {gqf }}(-\rightarrow-)$ which specialize to give $K$-theory (at least for smooth varieties), $K$-homology, and BorelMoore $K$-homology. Also in this section basic properties like functoriality and the existence of pairings are developed. In particular, we prove that each of the theories $\mathcal{K}_{\text {geom }}(-\rightarrow-)$ and $\mathcal{K}_{g q f}(-\rightarrow-)$ satisfies the axioms of a "bivariant theory" in the sense of Fulton-MacPherson [10]. (The proof of this is relegated to an appendix.)

In Section 4 we develop a technical tool for use later in the paper, but which may also have utility outside the context of this paper - namely, we extend Voevodsky's notion of a "pretheory" to that of a "presheaf with $K_{0}^{\oplus}$ transfers". As an example of the utility of these results, we mention that Andrei Suslin employs (a closely related version of) this notion in his forthcoming paper "On the Grayson Spectral Sequence", and indeed he uses some of the properties of presheaves with $K_{0}^{\oplus}$ transfers proven in Section 4 . (The results Suslin requires were also proven in the author's unpublished thesis [32].) In broad terms, we prove that many of the 
desirable properties of pretheories remain valid for presheaves with $K_{0}^{\oplus}$ transfers, and we prove that many functors arising in $K$-theory admit $K_{0}^{\oplus}$ transfers (although they are not pretheories). The specific key properties enjoyed by presheaves with $K_{0}^{\oplus}$ transfers used in this paper are listed in Corollary 4.23,

Beginning with Section [5] the major theorems of this paper are proven. The main goal of Section 5 itself is to prove Borel-Moore algebraic $K$-homology, $\mathcal{K}^{B M}$, satisfies the expected localization property (see Theorem [5.5). This is followed in Section $\left[6\right.$ with a proof that the bivariant theory $\mathcal{K}_{g q f}$ introduced in Section 3 satisfies a suitable version of the so-called "Gersten Conjecture" (see Theorem6.2). Also of note in this section is a proof that the motivic complexes involving $K_{0}$ groups of automorphisms defined by Grayson [14] are locally equivalent to those of Voevodsky (see Theorem 6.5).

The tools developed in Sections 5 and 6 are employed in Section 7 to prove that Borel-Moore algebraic $K$-homology with finite coefficients, $\mathcal{K}^{B M}(-; \mathbb{Z} / n)$, satisfies étale descent on the category of normal, Cohen-Macaulay quasi-projective $k$-varieties (see Theorems 7.6 and 7.8). As usual, we require that $n$ be relatively prime to the exponential characteristic of $k$. To relate $\mathcal{K}^{B M}$ with Bott-inverted $K$-theory with finite coefficients, a form of Poincaré duality is needed. Such a duality result is proven in Section 8 for all smooth varieties that have a "fundamental class" relative to $\mathcal{K}^{B M}$-theory with $\mathbb{Z} / n$ coefficients (see Theorem 8.13). In Section 9 we prove that all smooth, quasi-projective $k$-varieties have such fundamental classes (see Theorem 19.3), so that the duality result applies to all such varieties. Finally, using the results developed in the previous sections, we state and prove in Section 10 the main theorem of the paper, Theorem 10.2 - Thomason's theorem for varieties over algebraically closed fields.

The author thanks Eric Friedlander for innumerable correspondences over the past several years which led to the ideas contained in this paper. This paper also benefited significantly from conversations with Dan Grayson that led to shortenings and improvements of several of the arguments below. Finally, the author thanks the referee for several useful comments.

\section{Definitions}

Throughout this paper, $k$ denotes a ground field and by a $k$-scheme we mean a separated scheme essentially of finite type over $k$. We let $S c h / k$ denote the category whose objects are $k$-schemes and whose morphisms are separated morphisms of $k$ schemes

In this paper we consider a large number of exact categories and their associated $K$-theory spectra. We list many of these categories in the following two definitions so as to provide a handy reference for the remainder of the paper. The reader may wish to skip ahead to Section 3, referring back to the definitions given here as necessary.

Definition 2.1. For a $k$-scheme $X$, let $\mathcal{M}(X)$ denote the abelian category of coherent $\mathcal{O}_{X}$-modules and let $\mathcal{P}(X)$ denote the exact full subcategory of $\mathcal{M}(X)$ consisting of all locally free $\mathcal{O}_{X}$-modules.

Suppose $f: Y \rightarrow X$ is a morphism of $k$-schemes and let $U \subset Y$ be a locally closed subscheme (i.e., $U$ is an open subscheme of a closed subscheme of $Y$ ). We define the following exact categories: 
- $\mathcal{M}^{\text {fin }}(Y \stackrel{f}{\longrightarrow} X)$ is the abelian full subcategory of $\mathcal{M}(Y)$ consisting of those $M$ such that the induced morphism from the support of $M$, written $\operatorname{supp}(M)$, to $X$ is a finite morphism. (Briefly, we say $M$ is finite over $X$.)

- $\mathcal{P}^{f i n}(Y \stackrel{f}{\longrightarrow} X)$ is the exact full subcategory of $\mathcal{M}^{f i n}(Y \stackrel{f}{\longrightarrow} X)$ consisting of those $M$ which are $\mathcal{O}_{X}$-flat. (Briefly, we say such an $M$ is finite and flat over $X$.) Equivalently, an object $M$ of $\mathcal{M}^{f i n}(Y \stackrel{f}{\longrightarrow} X)$ belongs to $\mathcal{P}^{f i n}(Y \stackrel{f}{\longrightarrow} X)$ if and only if $f_{*} M$ is locally free on $X$.

- $\mathcal{M}^{q f}(Y \stackrel{f}{\longrightarrow} X)$ is the abelian full subcategory of $\mathcal{M}(Y)$ consisting of those $M$ such that the morphism $\operatorname{supp}(M) \rightarrow X$ is quasi-finite (i.e., has finite fibers).

- $\mathcal{P}^{q f}(Y \stackrel{f}{\longrightarrow} X)$ is the exact full subcategory of $\mathcal{M}^{q f}(Y \stackrel{f}{\longrightarrow} X)$ consisting of those $M$ which are $\mathcal{O}_{X}$-flat.

- $\mathcal{M}^{q f}((U, Y) \stackrel{f}{\longrightarrow} X)$ is full abelian subcategory of $\mathcal{M}^{q f}\left(U \stackrel{\left.f\right|_{U}}{\longrightarrow} X\right)$ consisting of those $M$ such that the map from the closure of $\operatorname{supp}(M)$ in $Y$ to $X$ is a quasi-finite morphism. Note that $\mathcal{M}^{q f}((U, Y) \stackrel{f}{\longrightarrow} X)=\mathcal{M}^{q f}\left((U, \bar{U}) \stackrel{\left.f\right|_{\bar{T}}}{\longrightarrow} X\right)$, where $\bar{U}$ is the closure of $U$ in $Y$.

- $\mathcal{P}^{q f}((U, Y) \stackrel{f}{\longrightarrow} X)$ is the exact full subcategory of $\mathcal{M}^{q f}((U, Y) \stackrel{f}{\longrightarrow} X)$ consisting of those $M$ which are $\mathcal{O}_{X}$-flat.

When the morphism from $Y$ to $X$ is clear from context, we write each of the above categories more simply as $\mathcal{M}^{\text {fin }}(Y / X), \mathcal{P}^{\text {fin }}(Y / X), \mathcal{M}^{q f}(Y / X), \mathcal{P}^{q f}(Y / X)$, $\mathcal{M}^{q f}((U, Y) / X)$, and $\mathcal{P}^{q f}((U, Y) / X)$.

To each of these exact categories we define its associated $K$-theory spectrum using Quillen's $Q$ construction [20]. These spectra are written as

$$
\begin{array}{ll}
\mathcal{G}^{f i n}(Y \stackrel{f}{\longrightarrow} X), \quad \mathcal{K}^{f i n}(Y \stackrel{f}{\longrightarrow} X), \quad \mathcal{G}^{q f}(Y \stackrel{f}{\longrightarrow} X), \\
\mathcal{K}^{q f}(Y \stackrel{f}{\longrightarrow} X), \quad \mathcal{G}^{q f}((U, Y) \stackrel{f}{\longrightarrow} X), \quad \mathcal{K}^{q f}((U, Y) \stackrel{f}{\longrightarrow} X),
\end{array}
$$

or, more briefly, as

$$
\mathcal{G}^{f i n}(Y / X), \mathcal{K}^{f i n}(Y / X), \mathcal{G}^{q f}(Y / X), \mathcal{K}^{q f}(Y / X), \mathcal{G}^{q f}((U, Y) / X), \mathcal{K}^{q f}((U, Y) / X) .
$$

The corresponding $n$-th homotopy group for each of these spectra is written as $G_{n}^{f i n}(Y / X), K_{n}^{f i n}(Y / X), G_{n}^{q f}(Y / X)$, etc.

In this paper, the primary example of a morphism $f: Y \rightarrow X$ arising in the above definitions occurs when $Y=X \times Z$ and $f=\pi_{1}$ is the canonical projection map. To simplify the notation in this case, we make the following definitions.

Definition 2.2. Let $X$ and $Y$ be $k$-schemes, let $\pi: X \times Y \rightarrow X$ denote the canonical projection map, and let $U$ be a locally closed subscheme of $Y$. Define exact categories as follows:

- $\mathcal{M}^{\text {fin }}(X, Y)=\mathcal{M}^{\text {fin }}(X \times Y \stackrel{\pi}{\longrightarrow} X)$,

- $\mathcal{P}^{f i n}(X, Y)=\mathcal{P}^{f i n}(X \times Y \stackrel{\pi}{\longrightarrow} X)$,

- $\mathcal{M}^{q f}(X, Y)=\mathcal{M}^{q f}(X \times Y \stackrel{\pi}{\longrightarrow} X)$,

- $\mathcal{P}^{q f}(X, Y)=\mathcal{P}^{q f}(X \times Y \stackrel{\pi}{\longrightarrow} X)$,

- $\mathcal{M}^{q f}(X,(U, Y))=\mathcal{M}^{q f}((X \times U, X \times Y) \stackrel{\pi}{\longrightarrow} X)$,

- $\mathcal{P}^{q f}(X,(U, Y))=\mathcal{P}^{q f}((X \times U, X \times Y) \stackrel{\pi}{\longrightarrow} X)$. 
The associated $K$-theory spectra are written as:

- $G^{f i n}(X, Y)=G^{f i n}(X \times Y \stackrel{\pi}{\longrightarrow} X)$,

- $\mathcal{K}^{\text {fin }}(X, Y)=\mathcal{K}^{\text {fin }}(X \times Y \stackrel{\pi}{\longrightarrow} X)$,

- $\mathcal{G}^{q f}(X, Y)=\mathcal{G}^{q f}(X \times Y \stackrel{\pi}{\longrightarrow} X)$,

- $\mathcal{K}^{q f}(X, Y)=\mathcal{K}^{q f}(X \times Y \stackrel{\pi}{\longrightarrow} X)$

- $\mathcal{G}^{q f}(X,(U, Y))=\mathcal{G}^{q f}((X \times U, X \times Y) \stackrel{\pi}{\longrightarrow} X)$,

- $\mathcal{K}^{q f}(X,(U, Y))=\mathcal{K}^{q f}((X \times U, X \times Y) \stackrel{\pi}{\longrightarrow} X)$.

The associated $K$-groups for each of these spectra are written using subscripts as usual.

The following lengthy proposition describes the functoriality of the various theories defined in Definition 2.1

Proposition 2.3. Let $Y \stackrel{h}{\longrightarrow} Z \stackrel{j}{\longrightarrow} X$ and $g: X^{\prime} \rightarrow X$ be morphisms of $k$-schemes. Define $f: Y \rightarrow X$ by $f=j \circ h$, define $Y^{\prime}=Y \times_{X^{\prime}} X$, and let $f^{\prime}: Y^{\prime} \rightarrow X^{\prime}$ be the induced map. We have the following natural maps of spectra which are induced by pullback or pushforward of coherent sheaves:

(1) $h_{*}: \mathcal{G}^{\text {fin }}(Y \stackrel{f}{\longrightarrow} X) \rightarrow \mathcal{G}^{\text {fin }}(Z \stackrel{j}{\longrightarrow} X)$,

(2) $g^{*}: \mathcal{G}^{\text {fin }}(Y \stackrel{f}{\longrightarrow} X) \rightarrow \mathcal{G}^{\text {fin }}\left(Y^{\prime} \stackrel{f^{\prime}}{\longrightarrow} X^{\prime}\right)$, provided $g$ is flat,

(3) $h_{*}: \mathcal{K}^{f i n}(Y \stackrel{f}{\longrightarrow} X) \rightarrow \mathcal{K}^{f i n}(Z \stackrel{j}{\longrightarrow} X)$,

(4) $g^{*}: \mathcal{K}^{f i n}(Y \stackrel{f}{\longrightarrow} X) \rightarrow \mathcal{K}^{f i n}\left(Y^{\prime} \stackrel{f^{\prime}}{\longrightarrow} X^{\prime}\right)$,

(5) $h^{*}: \mathcal{K}^{f i n}(Z \stackrel{j}{\longrightarrow} X) \rightarrow \mathcal{K}^{f i n}(Y \stackrel{f}{\longrightarrow} Z)$, provided $h$ is finite and flat,

(6) $h_{*}: \mathcal{G}^{q f}(Y \stackrel{f}{\longrightarrow} X) \rightarrow \mathcal{G}^{q f}(Z \stackrel{j}{\longrightarrow} X)$, provided $h$ is proper,

(7) $g^{*}: \mathcal{G}^{q f}(Y \stackrel{f}{\longrightarrow} X) \rightarrow \mathcal{G}^{q f}\left(Y^{\prime} \stackrel{f^{\prime}}{\longrightarrow} X^{\prime}\right)$, provided $g$ is flat,

(8) $h_{*}: \mathcal{K}^{q f}(Y \stackrel{f}{\longrightarrow} X) \rightarrow \mathcal{G}^{q f}(Z \stackrel{j}{\longrightarrow} X)$, provided $h$ is proper,

(9) $g^{*}: \mathcal{K}^{q f}(Y \stackrel{f}{\longrightarrow} X) \rightarrow \mathcal{K}^{q f}\left(Y^{\prime} \stackrel{f^{\prime}}{\longrightarrow} X^{\prime}\right)$,

(10) $h^{*}: \mathcal{K}^{q f}(Z \stackrel{j}{\longrightarrow} X) \rightarrow \mathcal{K}^{q f}(Y \stackrel{f}{\longrightarrow} X)$, provided $h$ is quasi-finite and flat.

Moreover, the maps of type (3) determine a covariant functor up to homotopy $(Y \rightarrow X) \mapsto \mathcal{K}^{\text {fin }}(Y / X)$ from $S c h / X$ (the category of $k$-schemes over $\left.X\right)$ to spectra, and the maps of type $(4)$ give $\left(X^{\prime} \rightarrow X\right) \mapsto \mathcal{K}^{f i n}\left(Y^{\prime} \stackrel{f^{\prime}}{\longrightarrow} X^{\prime}\right)$ the structure of a contravariant functor up to homotopy from $S c h / X$ to spectra. Similarly, the maps of type (8) determine a covariant functor on the category with objects in Sch/X and morphism given by proper morphisms, and the maps of type (9) determine a contravariant functor on $S c h / X$.

Proof. Each of these maps of spectra is induced by the evident corresponding functor, which in each case may readily be verified to be exact. The fact that $(Y \rightarrow$ $X) \mapsto \mathcal{K}^{f i n}(Y / X)$ is functorial follows from the fact that given $X^{\prime \prime} \stackrel{p}{\longrightarrow} X^{\prime} \stackrel{g}{\longrightarrow} X$, the functors $p^{*} \circ g^{*}$ and $(g \circ p)^{*}$ from $\mathcal{P}^{\text {fin }}(Y \rightarrow X)$ to $\mathcal{P}^{\text {fin }}\left(Y^{\prime \prime} \rightarrow X^{\prime \prime}\right)$ are naturally isomorphic. The other functoriality assertions hold for similar reasons.

Proposition 2.4. Let $Z \stackrel{g}{\longrightarrow} Y \stackrel{f}{\longrightarrow} X$ be morphisms of $k$-schemes and assume $U \longmapsto$ $Z$ is a locally closed subscheme. The functors

$$
\begin{gathered}
\mathcal{P}^{f i n}(Z \stackrel{g}{\longrightarrow} Y) \times \mathcal{P}^{f i n}(Y \stackrel{f}{\longrightarrow} X) \rightarrow \mathcal{P}^{f i n}(Z \stackrel{f \circ g}{\longrightarrow} X), \\
\mathcal{P}^{q f}(Z \stackrel{g}{\longrightarrow} Y) \times \mathcal{P}^{q f}(Y \stackrel{f}{\longrightarrow} X) \rightarrow \mathcal{P}^{q f}(Z \stackrel{f \circ g}{\longrightarrow} X),
\end{gathered}
$$


and

$$
\mathcal{P}^{q f}((U, Z) \stackrel{g}{\longrightarrow} Y) \times \mathcal{P}^{q f}(Y \stackrel{f}{\longrightarrow} X) \rightarrow \mathcal{P}^{q f}((U, Z) \stackrel{f \circ g}{\longrightarrow} X)
$$

each given by the formula $(M, N) \mapsto M \otimes_{\mathcal{O}_{Z}} g^{*} N$ are biexact and thus induce pairings

$$
\begin{aligned}
\mathcal{K}^{f i n}(Z \stackrel{g}{\longrightarrow} Y) \wedge \mathcal{K}^{f i n}(Y \stackrel{f}{\longrightarrow} X) \rightarrow \mathcal{K}^{f i n}(Z \stackrel{f \circ g}{\longrightarrow} X), \\
\mathcal{K}^{q f}(Z \stackrel{g}{\longrightarrow} Y) \wedge \mathcal{K}^{q f}(Y \stackrel{f}{\longrightarrow} X) \rightarrow \mathcal{K}^{q f}(Z \stackrel{f \circ g}{\longrightarrow} X),
\end{aligned}
$$

and

$$
\mathcal{K}^{q f}((U, Z) \stackrel{g}{\longrightarrow} Y) \wedge \mathcal{K}^{q f}(Y \stackrel{f}{\longrightarrow} X) \rightarrow \mathcal{K}^{q f}((U, Z) \stackrel{f \circ g}{\longrightarrow} X) .
$$

of spectra.

Proof. It is easy to verify that the targets of these functors are correct as stated (for example, that $M \in \mathcal{P}^{q f}(Z \stackrel{g}{\longrightarrow} Y)$ and $N \in \mathcal{P}^{q f}(Y \stackrel{f}{\longrightarrow} X)$ implies $M \otimes_{\mathcal{O}_{Z}} g^{*} N \in$ $\left.\mathcal{P}^{q f}(Z \stackrel{f \circ g}{\longrightarrow} X)\right)$. The flatness hypotheses ensure that these functors are biexact. The fact that such biexact functors leads to pairings of spectra is well known (and is worked out in careful detail in [8, C.2]).

The pairings of Proposition 2.4 determine the related pairings

$$
\begin{aligned}
& \mathcal{K}^{f i n}(Y, Z) \wedge \mathcal{K}^{f i n}(X, Y) \rightarrow \mathcal{K}^{f i n}(X, Z), \\
& \mathcal{K}^{q f}(Y, Z) \wedge \mathcal{K}^{q f}(X, Y) \rightarrow \mathcal{K}^{q f}(X, Z), \quad \text { if } Y \text { is projective, and } \\
& \mathcal{K}^{q f}(Y,(U, Z)) \wedge \mathcal{K}^{q f}(X, Y) \rightarrow \mathcal{K}^{q f}(X,(U, Z)), \quad \text { if } Y \text { is projective }
\end{aligned}
$$

by taking the composition of

$$
\begin{aligned}
\mathcal{K}^{f i n}(Y \times Z / Y) \wedge \mathcal{K}^{f i n}(X \times Y / X) \stackrel{\pi_{1}^{*} \wedge i d}{\longrightarrow} \mathcal{K}^{f i n}(X \times Y \times Z / X \times Y) \wedge \mathcal{K}^{f i n}(X \times Y / X) \\
\rightarrow \mathcal{K}^{f i n}(X \times Y \times Z / X) \stackrel{\left(\pi_{13}\right) *}{\longrightarrow} \mathcal{K}^{f i n}(X \times Z / X),
\end{aligned}
$$

and similarly for the other two cases. (The projective hypothesis is needed in the last two cases to ensure $\left(\pi_{13}\right)_{*}$ is defined.)

\section{Bivariant $K$-theory and ALgebraic $K$-Homology}

In this section we define the algebraic $K$-homology of a variety. Both algebraic $K$-homology and algebraic $K$-theory, at least for smooth varieties, are each special cases of a bivariant theory in the sense of Fulton-MacPherson [10] which is written $\mathcal{K}_{\text {geom }}(X \rightarrow Y)$ and which is also defined in this section. Since the constructions below do not recover algebraic $K$-theory for singular varieties (due to lack of homotopy invariance), we use the subscript geom in our notation. The motivation for this notation is that the space $\mathcal{K}_{\text {geom }}(X \times Y \rightarrow X)$ can be described as the "algebraic mapping space" from $X$ to a certain ind-variety parameterizing finite length coherent sheaves on $Y$ (see [15]), and is thus "geometric" in nature. (Since this description in terms of algebraic mapping spaces is not needed in this paper, we do not include it.) We also define a related bivariant theory, written $\mathcal{K}_{g q f}(X \rightarrow Y)$, defined in terms of sheaves with quasi-finite support.

Definition 3.1. Given a morphism $f: Y \rightarrow X$ of $k$-schemes, define

$$
\mathcal{K}_{\text {geom }}(Y \stackrel{f}{\longrightarrow} X)=\mathcal{K}_{\text {geom }}(Y / X)
$$


to be the spectrum defined as the geometric realization of the simplicial spectrum $d \mapsto \mathcal{K}^{\text {fin }}\left(Y \times \Delta^{d} / X \times \Delta^{d}\right)$.

We introduce the following shorthand for special cases of the above construction:

$$
\begin{aligned}
\mathcal{K}_{\text {geom }}(X) & =\mathcal{K}_{\text {geom }}(X / X)=\left|d \mapsto \mathcal{K}\left(X \times \Delta^{d}\right)\right|, \\
\mathcal{K}^{\text {hom }}(Y) & =\mathcal{K}_{\text {geom }}(Y / \operatorname{Spec} k)=\left|d \mapsto \mathcal{K}^{\text {fin }}\left(Y \times \Delta^{d} / \Delta^{d}\right)\right| .
\end{aligned}
$$

The spectrum $\mathcal{K}^{\text {hom }}(Y)$ is called the algebraic $K$-homology of $Y$.

We index the homotopy groups of these spectra as follows:

$$
\begin{aligned}
K_{\text {geom }}^{-n}(X / Y) & =\pi_{n} \mathcal{K}_{\text {geom }}(X / Y), \\
K_{\text {geom }}^{-n}(X) & =\pi_{n} \mathcal{K}_{\text {geom }}(X), \\
K_{n}^{\text {hom }}(Y) & =\pi_{n} \mathcal{K}^{\text {hom }}(Y) .
\end{aligned}
$$

More generally, if $A$ is an abelian group, we let $K_{\text {geom }}^{-n}(X / Y ; A), K_{\text {geom }}^{-n}(X ; A)$, $K_{n}^{\text {hom }}(Y ; A)$ denote the homotopy groups with coefficients in $A$ of the corresponding spectra.

Remark 3.2. Strictly speaking, the assignment $V \mapsto \mathcal{K}^{\text {fin }}(Y \times V / X \times V)$, as we have defined it above, is a contravariant functor only up to homotopy, since the assignment $V \mapsto \mathcal{P}^{\text {fin }}(Y \times V / X \times V)$ is not actually a functor, but merely a functor up to natural isomorphism. Here we describe how to make Definition 3.1 rigorous by constructing a degree-wise homotopy equivalent version of $\mathcal{K}^{\text {fin }}(Y \times-/ X \times-)$ that is a strictly functorial assignment of spectra to $k$-schemes. This is accomplished using the technique due to Grayson [13, §10] (see also [8, App. C]) to replace the exact categories $\mathcal{P}^{\text {fin }}(Y \times V / X \times V)$ with equivalent ones so that the resulting assignment $V \mapsto \mathcal{P}^{\text {fin }}(Y \times V / X \times V)$ is strictly functorial.

Specifically, redefine $\mathcal{P}^{f i n}(Y / X)$ to be the category in which an object is an assignment $\left(p: X^{\prime} \rightarrow X\right) \mapsto M_{p}$, where $p$ is an arrow in $S c h / X$ and $M_{p}$ is a coherent sheaf on $Y^{\prime}=X^{\prime} \times_{X} Y$ that is finite and flat over $X$ (i.e., $M_{p}$ is an object of the category we formerly wrote as $\left.\mathcal{P}^{\text {fin }}\left(Y^{\prime} / X^{\prime}\right)\right)$, together with isomorphisms $\theta_{p, q}$ : $\left(q \times i d_{Y}\right)^{*} M_{p} \stackrel{\cong}{\longrightarrow} M_{p \circ q}$, for every pair of composable morphisms $X^{\prime \prime} \stackrel{q}{\longrightarrow} X^{\prime} \stackrel{p}{\longrightarrow} X$. These isomorphisms are required to satisfy the conditions that $\theta_{p, i d}=i d$ and that given a triple of composable morphisms $X^{\prime \prime \prime} \stackrel{r}{\longrightarrow} X^{\prime \prime} \stackrel{q}{\longrightarrow} X^{\prime} \stackrel{p}{\longrightarrow} X$, the maps $\theta_{p \circ q, r} \circ$ $\left(r \times i d_{Y}\right)^{*}\left(\theta_{p, q}\right)$ and $\theta_{p, q \circ r}$ coincide under the canonical isomorphism

$$
\left(r \times i d_{Y}\right)^{*}\left(q \times i d_{Y}\right)^{*} M_{p} \cong\left(q \circ r \times i d_{Y}\right)^{*} M_{p} .
$$

A morphism $f$ in this newly defined category $\mathcal{P}^{\text {fin }}(Y / X)$ from $\left(p \mapsto M_{p}\right)$ to $(p \mapsto$ $N_{p}$ ) is given by a compatible family of morphisms $f_{p}: M_{p} \rightarrow N_{p}$. The resulting category $\mathcal{P}^{\text {fin }}(Y / X)$ is equivalent via the forgetful functor to the category formerly called by the same name, but now the assignment $X \mapsto \mathcal{P}^{\text {fin }}(Y / X)$ is strictly functorial in $X$, since the map $f^{*}: \mathcal{P}^{f i n}(Y / X) \rightarrow \mathcal{P}^{\text {fin }}\left(Y^{\prime} / X^{\prime}\right)$ associated to a morphism $f: X^{\prime} \rightarrow X$ is given by merely restricting the domain of the assignment $p \mapsto M_{p}$.

This construction also suffices to make the pushforward maps of Proposition 2.3 (3) strictly functorial. Specifically, given morphisms $Y \stackrel{g}{\longrightarrow} Z \rightarrow X$ and an object $M=\left(p \mapsto M_{p}\right)$ of $\mathcal{P}^{\text {fin }}(Y / X)$, define $g_{*} M$ to be the object of $\mathcal{P}^{\text {fin }}(Z / X)$ defined by the assignment $p \mapsto\left(i d_{X^{\prime}} \times g\right)_{*} M_{p}$ and the family of isomorphisms given by the 
compositions of the form

$$
\left(q \times 1_{Z}\right)^{*}\left(1_{X^{\prime}} \times g\right)_{*} M_{p} \cong\left(1_{X^{\prime \prime}} \times g\right)_{*}\left(q \times 1_{Y}\right)^{*} M_{p} \cong\left(1_{X^{\prime \prime}} \times g\right)_{*} M_{p \circ q} .
$$

(The first isomorphism here is the canonical one which results from the fact that $M_{p}$ is $\mathcal{O}_{X^{\prime}}$-flat and that the higher derived images of $\left(1_{X^{\prime}} \times g\right)_{*}$ and $\left(1_{X^{\prime \prime}} \times g\right)_{*}$ vanish due to the finiteness hypothesis.) One may verify that these rules define a functor $g_{*}: \mathcal{P}^{\text {fin }}(Y / X) \rightarrow \mathcal{P}^{\text {fin }}(Z / X)$. Finally, one may verify that given a morphism $f: X^{\prime} \rightarrow X$, the diagram

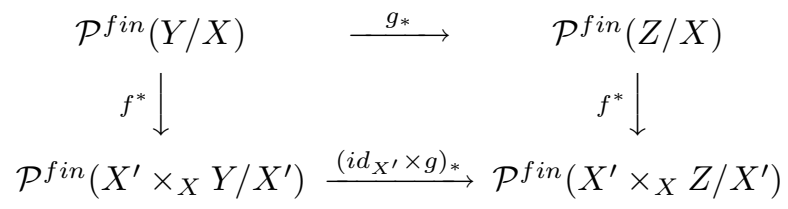

commutes exactly.

As shown in the following result, $\mathcal{K}_{\text {geom }}(X)$ is weakly equivalent to $\mathcal{K}(X)$, the usual algebraic $K$-theory of $X$ as defined by Quillen, provided $X$ is regular. More generally, there are natural maps $\mathcal{K}(X) \rightarrow \mathcal{K}_{\text {geom }}(X) \rightarrow \mathcal{K} H(X)$, where $\mathcal{K} H(X)$ denotes Weibel's "homotopy algebraic $K$-theory" [37], and the former map is a weak equivalence provided $X$ is $K_{0}$-regular, with the latter map a weak equivalence provided $X$ is $K_{0}$-regular and the negative algebraic $K$-groups of $X$ vanish. In this paper, the subscript geom serves as a reminder that $\mathcal{K}_{\text {geom }}$ deviates from $\mathcal{K}$ for singular varieties, but in fact we are ultimately only concerned with smooth varieties. Note also that we have taken the opportunity to rectify the unfortunate convention that the homotopy groups of $K$-theory are written using subscripts $\left(K_{n}(X)=\pi_{n} \mathcal{K}(X)\right)$, by using superscripts for the cohomology theory $\mathcal{K}_{\text {geom }}$.

Proposition 3.3. If $X$ is a regular $k$-scheme, then $\mathcal{K}_{\text {geom }}(X)$ is weakly equivalent to $\mathcal{K}(X)$, the usual algebraic $K$-theory spectrum of $X$.

Proof. The map of simplicial spectra

$$
\mathcal{K}(X) \rightarrow\left(d \mapsto \mathcal{K}^{\text {fin }}\left(X \times \Delta^{d} / X \times \Delta^{d}\right)\right),
$$

where the source is constant in the simplicial direction, is a degree-wise weak equivalence since $K$-theory is homotopy invariant on regular schemes.

Since we will mostly use the construction $\mathcal{K}_{\text {geom }}(Y / X)$ in the special case where $Y=X \times Z$, we introduce special notation in this setting:

Definition 3.4. For $k$-schemes $X$ and $Y$, define

$$
\mathcal{K}_{\text {geom }}(X, Y)=\mathcal{K}_{\text {geom }}(X \times Y / X)=\left|d \mapsto \mathcal{K}^{\text {fin }}\left(X \times Y \times \Delta^{d} / X \times \Delta^{d}\right)\right|,
$$

where $X \times Y \rightarrow X$ is the canonical projection, and let $K_{\text {geom }}^{-n}(X, Y ; A)$ denote its $n$-th homotopy group with coefficients in the abelian group $A$.

Note that we have the following identifications:

$$
\begin{aligned}
\mathcal{K}^{\text {hom }}(Y) & =\mathcal{K}_{\text {geom }}(\operatorname{Spec} k, Y), \\
\mathcal{K}_{\text {geom }}(X) & =\mathcal{K}_{\text {geom }}(X, \operatorname{Spec} k) .
\end{aligned}
$$

We now define a variation of $K_{\text {geom }}$, using coherent sheaves with quasi-finite support in place of coherent sheaves with finite support. One should view the resulting theory, $\mathcal{K}_{g q f}$, as the "Borel-Moore theory" associated to $\mathcal{K}_{\text {geom }}$. 
Definition 3.5. Given a morphism $f: Y \rightarrow X$ of $k$-schemes, the spectrum

$$
\mathcal{K}_{g q f}(Y \stackrel{f}{\longrightarrow} X)=\mathcal{K}_{g q f}(Y / X)
$$

is the geometric realization of the simplicial spectrum $d \mapsto \mathcal{K}^{q f}\left(Y \times \Delta^{d} / X \times \Delta^{d}\right)$.

The Borel-Moore K-homology (or algebraic K-homology with quasi-finite supports) of a quasi-projective $k$-variety $Y$ is defined to be $\mathcal{K}_{g q f}(Y / \operatorname{Spec} k)$ and is written

$$
\mathcal{K}^{B M}(Y)=\mathcal{K}_{g q f}(Y / \operatorname{Spec} k)=\left|d \mapsto \mathcal{K}^{q f}\left(Y \times \Delta^{d} / \Delta^{d}\right)\right| .
$$

More generally, if $U \longmapsto Y$ is a locally closed subscheme, we define

$$
\mathcal{K}_{g q f}((U, Y) / X)=\left|d \mapsto \mathcal{K}^{q f}\left(\left(U \times \Delta^{d}, Y \times \Delta^{d}\right) / X \times \Delta^{d}\right)\right| .
$$

Finally, with no map $Y \rightarrow X$ specified, we define

$$
\mathcal{K}_{\text {gqf }}(X, Y)=\mathcal{K}_{g q f}(X \times Y / X)
$$

and

$$
\mathcal{K}_{g q f}(X,(U, Y))=\mathcal{K}_{g q f}((X \times U, X \times Y) / X),
$$

where $X \times Y \rightarrow X$ is the canonical projection.

The homotopy groups of the above spectra are written

$$
\begin{aligned}
K_{n}^{\mathrm{BM}}(Y) & =\pi_{n} \mathcal{K}^{B M}(Y), \\
K_{g q f}^{-n}((U, Y) / X) & =\pi_{n} \mathcal{K}_{g q f}((U, Y) / X), \\
K_{g q f}^{-n}(X, Y) & =\pi_{n} \mathcal{K}_{g q f}(X, Y), \\
K_{g q f}^{-n}(X,(U, Y)) & =\pi_{n} \mathcal{K}_{g q f}(X,(U, Y)),
\end{aligned}
$$

and similarly for homotopy groups with coefficients in an abelian group $A$.

Note that the definition of $\mathcal{K}_{g q f}((U, Y) / X)$ subsumes $\mathcal{K}_{g q f}(Y / X)$ by taking $U=Y$. The theory $\mathcal{K}_{g q f}((U, Y) / X)$ is not actually a bivariant theory in the sense of Fulton-MacPherson, but rather serves as a useful technical construction for comparing related theories. (See especially Section 5.) As before, to render Definition 3.5 rigorous, one must use a strictly functorial model for $\mathcal{K}^{q f}((U \times-, Y \times-) / X \times-)$. This is accomplished by paralleling the construction of Remark 3.2 in the obvious manner.

Proposition 3.6. The spectrum $\mathcal{K}_{\text {geom }}(Y \rightarrow X)$ is a covariant functor on $S c h / X$ via the pushforward maps of Proposition [2.3(3). For a fixed morphism $Y \rightarrow X$, sending a morphism $f: X^{\prime} \rightarrow X$ to $\mathcal{K}_{\text {geom }}\left(X^{\prime} \times_{X} Y \rightarrow X^{\prime}\right)$ defines a contravariant functor on $S c h / X$ using the pullback maps of Proposition [2.3 4).

In particular, $\mathcal{K}_{\text {geom }}(X, Y)$ is contravariantly functorial in $X$. The spectrum $\mathcal{K}_{\text {geom }}(X, Y)$ is also covariantly functorial in $Y$, where a morphism $g: Y \rightarrow Z$ is sent to the map

$(i d \times g)_{*}: \mathcal{K}_{\text {geom }}(X, Y)=\mathcal{K}_{\text {geom }}(X \times Y / X) \rightarrow \mathcal{K}_{\text {geom }}(X \times Z / X)=\mathcal{K}_{\text {geom }}(X, Z)$.

Proof. The results follow from Remark 3.2 which establishes that the squares

$$
\begin{array}{ccc}
\mathcal{K}^{f i n}\left(Y \times \Delta^{d} / X \times \Delta^{d}\right) & \stackrel{(1 \times \alpha)^{*}}{\longrightarrow} & \mathcal{K}^{f i n}\left(Y \times \Delta^{e} / X \times \Delta^{e}\right) \\
(f \times 1)^{*} \downarrow & (f \times 1)^{*} \downarrow \\
\mathcal{K}^{f i n}\left(X^{\prime} \times{ }_{X} Y \times \Delta^{d} / X^{\prime} \times \Delta^{d}\right) & \stackrel{(1 \times \alpha)^{*}}{\longrightarrow} \mathcal{K}^{f i n}\left(X^{\prime} \times{ }_{X} Y \times \Delta^{e} / X^{\prime} \times \Delta^{e}\right)
\end{array}
$$


and

$$
\begin{array}{cc}
\mathcal{K}^{f i n}\left(Y \times \Delta^{d} / X \times \Delta^{d}\right) & \stackrel{(1 \times \alpha)^{*}}{\longrightarrow} \mathcal{K}^{f i n}\left(Y \times \Delta^{e} / X \times \Delta^{e}\right) \\
\quad(g \times 1)_{*} \downarrow & (g \times 1)_{*} \downarrow \\
\mathcal{K}^{f i n}\left(Z \times \Delta^{d} / X \times \Delta^{d}\right) \stackrel{(1 \times \alpha)^{*}}{\longrightarrow} \mathcal{K}^{f i n}\left(Z \times \Delta^{e} / X \times \Delta^{e}\right)
\end{array}
$$

commute on the nose for any face or degeneracy map $\alpha: \Delta^{e} \rightarrow \Delta^{d}$. Thus, the families of maps $\left(f \times 1_{\Delta^{d}}\right)^{*}$ and $\left(g \times 1_{\Delta^{d}}\right)_{*}$ determine maps of simplicial spectra

$$
f^{*}: \mathcal{K}_{\text {geom }}(Y / X) \rightarrow \mathcal{K}_{\text {geom }}\left(X^{\prime} \times_{X} Y / X^{\prime}\right)
$$

and

$$
g_{*}: \mathcal{K}_{\text {geom }}(Y / X) \rightarrow \mathcal{K}_{\text {geom }}\left(Z / X^{\prime}\right)
$$

Functoriality of these maps is easily verified.

In particular $\mathcal{K}^{\text {hom }}(Y)$ is a homology theory in the sense that it is covariantly functorial with respect to all maps. Thus it obviously differs from $G$-theory (also known as $K^{\prime}$-theory), since the latter has the same functoriality as Borel-Moore homology. But the two theories do not agree even on projective varieties, as the following result shows. (The proposition is proven by use of some of the results of Sections 4 and 5 below - we have included it here for the sake of motivation.)

Proposition 3.7. For a smooth, projective $k$-variety $Y$, there is a natural isomorphism

$$
K_{0}^{h o m}(Y) \cong C H_{0}(Y)
$$

where $\mathrm{CH}_{0}(Y)$ is the group of zero-cycles on $Y$ modulo rational equivalence.

Proof. Note that $K_{0}^{\text {hom }}(Y)$ is isomorphic to the cokernel of

$$
K_{0}^{f i n}\left(Y \times \mathbb{A}^{1} / \mathbb{A}^{1}\right) \stackrel{i_{0}^{*}-i_{1}^{*}}{\longrightarrow} K_{0}^{f i n}(Y / \operatorname{Spec} k),
$$

where $i_{\epsilon}: \operatorname{Spec} k \longmapsto \mathbb{A}^{1}$ is inclusion at $\epsilon$. As in Proposition 4.5 below, there is a natural cycle class map forming the horizontal maps of the commutative diagram

$$
\begin{array}{ll}
K_{0}^{f i n}\left(Y \times \mathbb{A}^{1} / \mathbb{A}^{1}\right) \stackrel{c y c}{\longrightarrow} c_{\text {equi }}^{0}\left(Y \times \mathbb{A}^{1} / \mathbb{A}^{1}\right) \\
i_{0}^{*}-i_{1}^{*} \downarrow \\
K_{0}^{f i n}(Y / \operatorname{Spec} k) \stackrel{\text { cyc }}{\longrightarrow} c_{\text {equi }}^{0}(Y / \operatorname{Spec} k) .
\end{array}
$$

(Here, $c_{\text {equi }}^{0}(Y \times X / X)$ is the free abelian group on closed, integral subschemes of $Y \times X$ which are finite and dominant over $X$.) Moreover, the proof of Proposition 5.4 below shows that the natural maps

$K_{0}^{f i n}\left(Y \times \mathbb{A}^{1} / \mathbb{A}^{1}\right) \stackrel{\cong}{\longrightarrow} G_{0}^{f i n}\left(Y \times \mathbb{A}^{1} / \mathbb{A}^{1}\right) \quad$ and $\quad K_{0}^{f i n}(Y /$ Spec $k) \stackrel{\cong}{\longrightarrow} G_{0}^{f i n}(Y / \operatorname{Spec} k)$

are isomorphisms. An easy devissage argument shows that $G_{0}^{f i n}(Y / \operatorname{Spec} k)$ is isomorphic to $c_{\text {equi }}^{0}(Y / \operatorname{Spec} k$ ) (which is the free abelian group on the set of closed points of $Y$ ), so that the bottom arrow of (2) is an isomorphism. One may readily verify that $c y c: G_{0}^{f i n}\left(Y \times \mathbb{A}^{1} / \mathbb{A}^{1}\right) \rightarrow c_{\text {equi }}^{0}\left(Y \times \mathbb{A}^{1} / \mathbb{A}^{1}\right)$ is split by the map sending $Z \subset Y \times \mathbb{A}^{1}$ to $\left[\mathcal{O}_{Z}\right]$, and thus is a surjection. It follows that the top arrow of (2) is surjective, and thus an easy diagram chase shows that $K_{0}^{\text {hom }}(Y)$ is isomorphic to the cokernel of the right-hand vertical map of (2). The fact that the latter is isomorphic to $\mathrm{CH}_{0}(Y)$ follows from [9, 4.4 and 8.1] and [26, 2.1]. 
Proposition 3.8. The maps of Proposition [2.3 8) give the assignment $(Y \rightarrow X) \mapsto$ $\mathcal{K}_{g q f}(Y \rightarrow X)$ the structure of a covariant functor on the category consisting of the objects of $S c h / X$ and morphisms given by proper morphisms of varieties over $X$.

Similarly, the maps of Proposition 2.3(10) give the assignment $(Y \rightarrow X) \mapsto$ $\mathcal{K}_{g q f}(Y \rightarrow X)$ the structure of a contravariant functor on the category with objects Sch $/ X$ and morphisms given by quasi-finite and flat morphisms of varieties over $X$.

Finally, for a fixed $Y \rightarrow X$, sending $f: X^{\prime} \rightarrow X$ to $\mathcal{K}_{g q f}\left(X^{\prime} \times_{X} Y \rightarrow X^{\prime}\right)$ is a contravariant functor on $S c h / X$ via the maps induced by those of Proposition 2.3 (9).

In particular, $\mathcal{K}^{B M}(Y)$ is covariantly functorial in $Y$ for proper morphisms and contravariantly functorial in $Y$ for quasi-finite, flat morphisms.

Proof. The proof is the same as that of Proposition 3.6

Proposition 3.9. Let $Z \stackrel{g}{\longrightarrow} Y \stackrel{f}{\longrightarrow} X$ be morphisms of $k$-varieties. The pairings of Proposition 2.4 induce pairings

$$
-\bullet-: \mathcal{K}_{\text {geom }}(Z \stackrel{g}{\longrightarrow} Y) \wedge \mathcal{K}_{\text {geom }}(Y \stackrel{f}{\longrightarrow} X) \rightarrow \mathcal{K}_{\text {geom }}(Z \stackrel{f \circ g}{\longrightarrow} X)
$$

and

$$
-\bullet-: \mathcal{K}_{g q f}(Z \stackrel{g}{\longrightarrow} Y) \wedge \mathcal{K}_{g q f}(Y \stackrel{f}{\longrightarrow} X) \rightarrow \mathcal{K}_{g q f}(Z \stackrel{f \circ g}{\longrightarrow} X)
$$

which are associative in the following sense: If $W \stackrel{h}{\longrightarrow} Z$ is another morphism of $k$-schemes, the diagrams

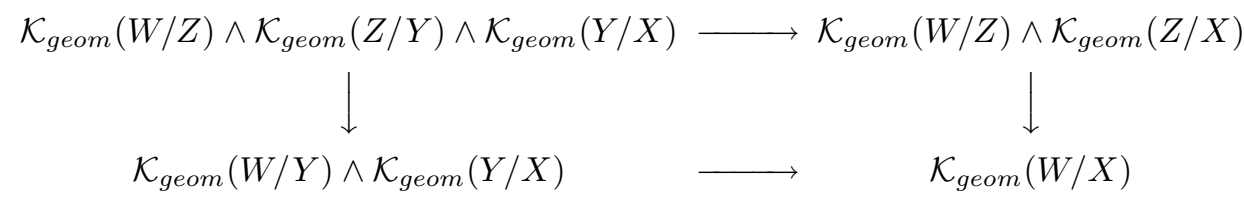

and

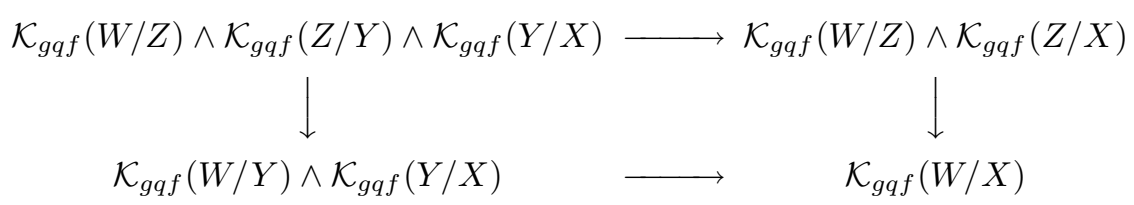

commute up to homotopy.

Proof. The collection of biexact pairings

$$
\mathcal{P}^{f i n}(Z \stackrel{g}{\longrightarrow} Y) \times \mathcal{P}^{f i n}(Y \stackrel{f}{\longrightarrow} X) \rightarrow \mathcal{P}^{f i n}(Z \stackrel{f \circ g}{\longrightarrow} X)
$$

of Proposition 2.4 extend to strictly functorial pairings in $X$ using the models for these categories described in Remark 3.2 Namely, if $p \mapsto M_{p}$ and $q \mapsto N_{q}$ are objects of $\mathcal{P}^{\text {fin }}(Z / Y)$ and $\mathcal{P}^{\text {fin }}(Y / X)$, then the assignment of $r: X^{\prime} \rightarrow X$ to $M_{r^{\prime}} \otimes_{\mathcal{O}_{Z}} g^{*} N_{h}$ (where $r^{\prime}: Y^{\prime} \rightarrow Y$ is the pullback of $r$ ) defines an object of $\mathcal{P}^{\text {fin }}(Z / X)$. It follows that the maps

$\mathcal{K}^{f i n}\left(Z \times \Delta^{d} / Y \times \Delta^{d}\right) \wedge \mathcal{K}^{f i n}\left(Y \times \Delta^{d} / X \times \Delta^{d}\right) \rightarrow \mathcal{K}^{f i n}\left(Z \times \Delta^{d} / X \times \Delta^{d}\right), \quad d \geq 0$,

determine a map of simplicial spectra. The geometric realization of this map is the map we seek. The asserted functoriality follows from the fact that tensor product 
is associative up to canonical isomorphism, which determines for each fixed $d \geq 0$ a homotopy joining the two evident maps of the form

$$
\begin{aligned}
\mathcal{K}^{f i n}\left(W \times \Delta^{d} / Z \times \Delta^{d}\right) & \wedge \mathcal{K}^{f i n}\left(Z \times \Delta^{d} / Y \times \Delta^{d}\right) \wedge \mathcal{K}^{f i n}\left(Y \times \Delta^{d} / X \times \Delta^{d}\right) \\
& \longrightarrow \mathcal{K}^{f i n}\left(W \times \Delta^{d} / X \times \Delta^{d}\right) .
\end{aligned}
$$

The canonical nature of the isomorphism used to define these homotopies implies that these homotopies commute with face and degeneracy maps, thereby determining a homotopy joining the two evident simplicial maps of the form

$$
\begin{aligned}
\mathcal{K}^{f i n}\left(W \times \Delta^{\bullet} / Z \times \Delta^{\bullet}\right) & \wedge \mathcal{K}^{f i n}\left(Z \times \Delta^{\bullet} / Y \times \Delta^{\bullet}\right) \wedge \mathcal{K}^{f i n}\left(Y \times \Delta^{\bullet} / X \times \Delta^{\bullet}\right) \\
& \longrightarrow \mathcal{K}^{f i n}\left(W \times \Delta^{\bullet} / X \times \Delta^{\bullet}\right) .
\end{aligned}
$$

The result involving $\mathcal{K}_{g q f}$ is proved in a parallel fashion.

Taking homotopy groups with coefficients in $\mathbb{Z} / n$ with $n$ any integer such that $n \not \equiv 2(\bmod 4)$ (possibly equal to 0$)$, the pairings of Proposition 3.9 induce pairing of abelian groups:

$$
\begin{gathered}
K_{\text {geom }}^{p}(Z / Y ; \mathbb{Z} / n) \otimes K_{\text {geom }}^{q}(Y / X ; \mathbb{Z} / n) \rightarrow K_{\text {geom }}^{p+q}(Z / X ; \mathbb{Z} / n), \\
K_{\text {gqf }}^{p}(Z / Y ; \mathbb{Z} / n) \otimes K_{\text {gqf }}^{q}(Y / X ; \mathbb{Z} / n) \rightarrow K_{\text {gqf }}^{p+q}(Z / X ; \mathbb{Z} / n) .
\end{gathered}
$$

However, the mod- $n$ Moore space fails have an appropriate coproduct rule if the prime factorization of $n$ involves small powers of 2 and 3. (In fact, the above pairings fail to exist even if $n \equiv 2(\bmod 4)$.) Thus, when using the product pairings (3)), we frequently assume that $n$ satisfies the following technical condition mentioned also in the introduction:

(Condition 2-3)

If $3 \mid n$, then $9 \mid n$, and if $2 \mid n$, then $16 \mid n$.

(See [27 A.6].) For such $n$, the pairings (3) are associative in the evident sense, and when $X=Y=Z$, these pairings are graded-commutative.

For any value of $n$, the maps of Propositions 3.6 and 3.8 induce functorial maps of abelian groups:

$$
\begin{aligned}
& f_{*}: K_{\text {geom }}^{p}(Y / X ; \mathbb{Z} / n) \rightarrow K_{\text {geom }}^{p}(Z / X ; \mathbb{Z} / n), \quad f: Y \rightarrow Z \text { arbitrary, } \\
& g^{*}: K_{\text {geom }}^{p}(Y / X ; \mathbb{Z} / n) \rightarrow K_{\text {geom }}^{p}\left(Y^{\prime} / X^{\prime} ; \mathbb{Z} / n\right), \quad g: X^{\prime} \rightarrow X \text { arbitrary, } \\
& f_{*}: K_{\text {gqf }}^{p}(Y / X ; \mathbb{Z} / n) \rightarrow K_{\text {gqf }}^{p}(Z / X ; \mathbb{Z} / n), \quad f: Y \rightarrow Z \text { proper, } \\
& g^{*}: K_{\text {gqf }}^{p}(Y / X ; \mathbb{Z} / n) \rightarrow K_{\text {gqf }}^{p}\left(Y^{\prime} / X^{\prime} ; \mathbb{Z} / n\right) \quad g: X^{\prime} \rightarrow X \text { arbitrary. }
\end{aligned}
$$

We also have special pullback maps of the form

(5) $f^{*}: K_{g q f}^{p}(Z / X ; \mathbb{Z} / n) \rightarrow K_{g q f}^{p}(Y / X ; \mathbb{Z} / n), \quad$ if $f: Y \rightarrow Z$ is quasi-finite, flat.

Using the results established above, it is not difficult to verify that the groups $K_{\text {geom }}^{*}$ and $K_{g q f}^{*}$ define bivariant theories in the sense of Fulton-MacPherson [10]. A bivariant theory assigns a graded abelian group to each morphism of the category $S c h / k$ together with pullback maps for arbitrary morphism and pushforward maps for "confined" morphisms. The definition of the class of confined morphisms is part of the definition of a bivariant theory, as is the class of so-called "independent squares", each of which is required to satisfy certain axioms (cf. [10, 2.1]). The proof of the following theorem along with a precise list of the Fulton-MacPherson axioms is contained in the appendix of this paper. 
Theorem 3.10. Let $n$ be an integer (possibly 0) such that $n$ satisfies (Condition $2-3)$.

The collection of graded abelian groups $K_{\text {geom }}^{*}(Y \stackrel{f}{\longrightarrow} X ; \mathbb{Z} / n)$, where $f: Y \rightarrow X$ ranges over all morphisms of $S c h / k$, together with the product pairings (3) and pushforward and pullback maps (4) satisfy the axioms of a skew-commutative bivariant theory with units in the sense of Fulton-MacPherson [10. In this case, the confined morphisms are all morphisms of $S c h / k$ and the independent squares are all pullback squares in $S c h / k$.

Similarly, the collection of graded abelian groups $K_{g q f}^{*}(X \stackrel{f}{\longrightarrow} Y ; \mathbb{Z} / n)$ forms a skew-commutative bivariant theory with units in the sense of Fulton-MacPherson, where the confined morphisms are all proper morphisms in $S c h / k$ and the independent squares are all pullback squares.

Moreover, the canonical maps

$$
K_{\text {geom }}^{*}(X \stackrel{f}{\longrightarrow} Y ; \mathbb{Z} / n) \rightarrow K_{\text {gqf }}^{*}(X \stackrel{f}{\longrightarrow} Y ; \mathbb{Z} / n),
$$

induced by the evident inclusions of categories, define a so-called "Grothendieck" transformation of bivariant theories.

As with any bivariant theory, we have cup and cap product operations. Explicitly, cup product for $K_{\text {geom }}^{*}$ is the pairing

$$
-\cup-: K_{\text {geom }}^{p}(X ; \mathbb{Z} / n) \otimes K_{\text {geom }}^{q}(X ; \mathbb{Z} / n) \rightarrow K_{\text {geom }}^{p+q}(X ; \mathbb{Z} / n)
$$

given by taking $X=Y=Z$ in (3). The analogous definition for $K_{g q f}^{*}$ leads to the exact same pairing since $\mathcal{K}_{g q f}(X / X)=\mathcal{K}_{\text {geom }}(X / X)=\mathcal{K}_{\text {geom }}(X)$. The cap product pairings for $K_{\text {geom }}^{*}$ and $K_{g q f}^{*}$ are given by the maps

$$
-\cap-: K_{\text {geom }}^{p}(Y ; \mathbb{Z} / n) \otimes K_{q}^{\mathrm{hom}}(Y ; \mathbb{Z} / n) \rightarrow K_{q-p}^{\mathrm{hom}}(Y ; \mathbb{Z} / n)
$$

and

$$
K_{\text {geom }}^{p}(Y ; \mathbb{Z} / n) \otimes K_{q}^{\mathrm{BM}}(Y ; \mathbb{Z} / n) \rightarrow K_{q-p}^{\mathrm{BM}}(Y ; \mathbb{Z} / n)
$$

defined by taking $Z=Y$ and $X=\operatorname{Spec} k$ in (3).

Additionally, as an immediate consequence of the axioms, we obtain natural Gysin maps. Namely, if $f: X \rightarrow Y$ is finite and flat, then $\mathcal{O}_{X}$ determines a class $[f]$ in $K_{\text {geom }}^{0}(X \rightarrow Y)$ and pairing with this class defines a "wrong-way" map

$$
f_{!}: K_{\text {geom }}^{*}(X) \rightarrow K_{\text {geom }}^{*}(Y)
$$

defined by $f_{!}(x)=f_{*}(x \bullet[f])$. Similarly, $[f]$ defines a map

$$
f^{!}: K_{*}^{\mathrm{hom}}(Y) \rightarrow K_{*}^{\mathrm{hom}}(X)
$$

by $f^{!}(y)=[f] \bullet y$. If $f: X \rightarrow Y$ is merely quasi-finite and flat, we have a similarly defined map

$$
f^{!}: K_{*}^{\mathrm{BM}}(Y) \rightarrow K_{*}^{\mathrm{BM}}(X) .
$$

These Gysin maps satisfy the expected axioms (cf. [10, 2.5]). Moreover, the last of these Gysin maps coincides with the map $f^{*}: K_{*}^{\mathrm{BM}}(Y) \rightarrow K_{*}^{\mathrm{BM}}(X)$ given in (5) (by taking $X=\operatorname{Spec} k, Y=X$, and $Z=Y$ ).

As shown in [10, $\S 2.3-2.6]$, the axioms of a bivariant theory directly imply a host on other useful properties. We list those such properties needed in this paper in the following result. 
Corollary 3.11. Let $n \in \mathbb{Z}$ be an integer (possibly 0) satisfying (Condition 2-3). Then

(1) $K_{\text {geom }}^{*}(X ; \mathbb{Z} / n)$ is a graded-commutative ring under cup product, and for any morphism $f: X^{\prime} \rightarrow X$, the pullback morphism $f^{*}$ is a ring homomorphism;

(2) $K_{*}^{\text {hom }}(X ; \mathbb{Z} / n)$ is a $K_{\text {geom }}^{*}(X ; \mathbb{Z} / n)$-module under cap product, and for any morphism $f: X \rightarrow Y$, the map

$$
f_{*}: K_{*}^{h o m}(X ; \mathbb{Z} / n) \rightarrow K_{*}^{h o m}(Y ; \mathbb{Z} / n)
$$

is a $K_{\text {geom }}^{*}(Y ; \mathbb{Z} / n)$-module homomorphism (where $K_{*}^{\text {hom }}(X ; \mathbb{Z} / n)$ is viewed as a $K_{\text {geom }}^{*}(Y ; \mathbb{Z} / n)$-module via restriction of scalars along $\left.f^{*}\right)$.

(3) $K_{*}^{B M}(X ; \mathbb{Z} / n)$ is a $K_{\text {geom }}^{*}(X ; \mathbb{Z} / n)$-module under cap product, and if $f$ : $X \rightarrow Y$ is proper,

$$
f_{*}: K_{*}^{B M}(X ; \mathbb{Z} / n) \rightarrow K_{*}^{B M}(Y ; \mathbb{Z} / n)
$$

is a $K_{\text {geom }}^{*}(Y ; \mathbb{Z} / n)$-module homomorphism.

(4) If $f: X \rightarrow Y$ is quasi-finite and flat, then the pullback map

$$
f^{*}: K_{*}^{B M}(Y ; \mathbb{Z} / n) \rightarrow K_{*}^{B M}(X ; \mathbb{Z} / n)
$$

defined in Proposition 3.8 is a $K_{\text {geom }}^{*}(Y ; \mathbb{Z} / n)$-module homomorphism.

Proof. The first three of these properties is a formal consequence of the axioms, as explained in [10, §2.3]. To prove the final assertion, since the map $f^{*}$ coincides with the "Gysin" homomorphism $f$ ! introduced above, the desired result is given by [10, $\left.\mathrm{G}_{3}(\mathrm{iii})\right]$.

For use in later sections, we record the following evident fact.

Proposition 3.12. If $f: Y \rightarrow X$ is a proper morphism of $k$-schemes, we have $\mathcal{M}^{\text {fin }}(Y / X)=\mathcal{M}^{\text {qf }}(Y / X)$ and $\mathcal{P}^{\text {fin }}(Y / X)=\mathcal{P}^{q f}(Y / X)$ and so we have $\mathcal{G}^{\text {fin }}(Y / X)$ $=\mathcal{G}^{q f}(Y / X)$ and $\mathcal{K}^{f i n}(Y / X)=\mathcal{K}^{q f}(Y / X)$. In particular, $\mathcal{K}_{g q f}(Y / X)=$ $\mathcal{K}_{\text {geom }}(Y / X)$ for such an $f$, and $\mathcal{K}^{B M}(Y)=\mathcal{K}^{\text {hom }}(Y)$ if $Y$ is any projective $k$ scheme.

Proof. A subscheme $Z$ of $Y$ which is quasi-finite over $X$ is necessarily finite, since $Y \rightarrow X$ is a proper morphism.

\section{Transfers}

In this section we develop and study a notion of transfers for presheaves on the category $S m / k$ of smooth, quasi-projective $k$-varieties. The notion of transfers studied here is analogous to (but slightly more general than) Voevodsky's notion of a pretheory [29, §3]. Some of the ideas in this section appeared in the author's thesis 32 and all of the ideas are motivated by the work of Voevodsky [29] and Suslin-Voevodsky [24. With an eye toward possible future applications, we endeavor to define transfer maps so as to include the largest collection of presheaves possible. This leads to the notion of a "presheaf with $K_{0}^{\oplus}$ transfers" defined below. In Suslin's forthcoming preprint "On the Grayson Spectral Sequence", he uses (a slightly weaker version of) presheaves with $K_{0}^{\oplus}$ transfers as part of his proof that the $E_{2}$ terms of Grayson's spectral sequence [14] coincide with the motivic cohomology groups. Thus, the results in this section also find applications beyond this paper. (See Remark 4.3.) 
Given a morphism $f: Y \rightarrow X$ in $S c h / k$, let $K_{0}^{f i n, \oplus}(Y / X)$ denote the Grothendieck group of $\mathcal{P}^{\text {fin }}(Y / X)$ regarded as merely an additive category. That is, $K_{0}^{f i n, \oplus}(Y / X)$ is the group completion of the abelian monoid generated by isomorphism classes of objects of $\mathcal{P}^{\text {fin }}(Y / X)$ subject to the relation $[M]+[N]=[P]$ if $P \cong M \oplus N$. (Note that not every short exact sequence of $\mathcal{P}^{f i n}(Y / X)$ splits even if $X$ and $Y$ are both affine varieties.) The groups $K_{0}^{f i n, \oplus}(Y / X)$ enjoy the same functoriality as do the groups $K_{0}^{f i n}(Y / X)$.

Definition 4.1 (Compare with Voevodsky's definition of a pretheory [29, 3.1]). A presheaf with $K_{0}^{\oplus}$ transfers is a presheaf $F$ of abelian groups defined on the category $S m / k$ equipped with the following additional structure: given a morphism $\pi: X \rightarrow S$ that is smooth of relative dimension one with $X$ and $S$ objects of $S m / k$ (for short, we will refer to $\pi$ as a "smooth curve"), there is a pairing of abelian groups

$$
\theta_{X / S}: K_{0}^{f i n, \oplus}(X / S) \otimes_{\mathbb{Z}} F(X) \rightarrow F(S) .
$$

These pairings are required to satisfy the following axioms:

(1) (Normalization) If $i: S \longmapsto X$ is a splitting of $\pi$ and $[i]=\left[\mathcal{O}_{\Gamma_{i}}\right] \in$ $K_{0}^{f i n, \oplus}(X / S)$ is the class of the structure sheaf of the graph of $i$, then $\theta_{X / S}([i] \otimes-): F(X) \rightarrow F(S)$ coincides with $i^{*}$.

(2) (Naturality) If $f: S^{\prime} \rightarrow S$ is any morphism of smooth varieties, $X^{\prime}=$ $X \times{ }_{S} S^{\prime}$, and $g: X^{\prime} \rightarrow X$ and $f^{*}: K_{0}^{f i n, \oplus}(X / S) \rightarrow K_{0}^{f i n, \oplus}\left(X^{\prime} / S^{\prime}\right)$ are the induced maps, then the diagram

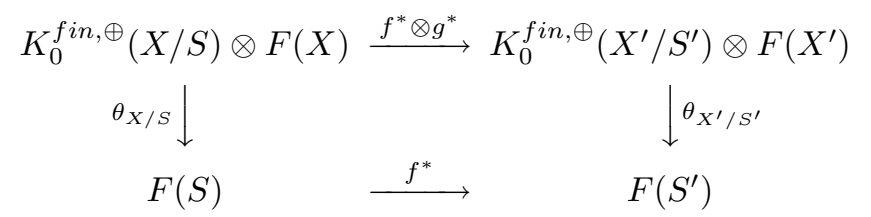

commutes.

(3) (Additivity) For $X$ and $Y$ in $S m / k$, the natural map $F(X \amalg Y) \rightarrow F(X) \oplus$ $F(Y)$ is an isomorphism.

A morphism $f: F \rightarrow G$ of presheaves with $K_{0}^{\oplus}$ transfers is a morphism of presheaves such that the diagram

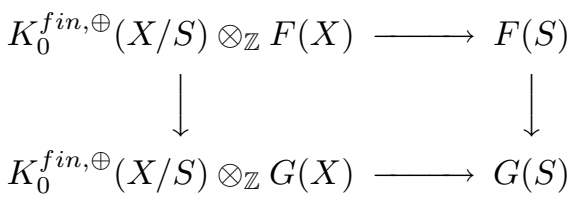

commutes for all smooth curves $X \rightarrow S$.

In general, given a presheaf $F$, a smooth curve $X \rightarrow S$ and an element $\alpha \in$ $K_{0}^{f i n, \oplus}(X / S)$, we let $\alpha_{*}: F(X) \rightarrow F(S)$ denote the map $\theta_{X / S}(\alpha \otimes-)$.

Definition 4.2. A presheaf with $K_{0}$ transfers is a presheaf $F$ of abelian groups on $S m / k$ satisfying the same axioms as in Definition 4.1 with $K_{0}^{f i n, \oplus}$ replaced everywhere by $K_{0}^{f i n}$.

Remark 4.3. In Suslin's forthcoming preprint "On the Grayson Spectral Sequence", he uses the notion of a " $K_{0}^{\oplus}$-presheaf". This is a presheaf $F$ on $S m / k$ equipped 
with additional pairings

$$
F(V) \otimes K_{0}^{f i n, \oplus}(U, V) \rightarrow F(U)
$$

satisfying certain evident axioms. (Here, $K_{0}^{f i n, \oplus}(U, V)=K_{0}^{f i n, \oplus}(U \times V / U)$.) In fact, these axioms amount to asserting that $F$ is a presheaf on $K^{\oplus}(S m / k)$, where $K^{\oplus}(S m / k)$ is the category whose objects are the objects of $S m / k$ but whose morphisms are given by the abelian groups $K^{\oplus}(X, Y)$. One may readily verify that by associating to each smooth curve $X \rightarrow S$ the map $K^{f i n, \oplus}(X / S) \rightarrow K^{\oplus}(S, X)$ induced by pushforward along $X \longmapsto S \times X$, every $K_{0}^{\oplus}$-presheaf is naturally a presheaf with $K^{\oplus}$ transfers. Thus, all of the results in this section about presheaves with $K_{0}^{\oplus}$ transfers can be employed in Suslin's context to $K^{\oplus}$-presheaves.

Observe that the canonical map $K_{0}^{f i n, \oplus}(X / S) \rightarrow K_{0}^{f i n}(X / S)$ is a surjection and represents a natural transformation of functors on the category of smooth schemes over $S$. (By a "smooth scheme over $S$ ", we mean here and elsewhere a morphism $Y \rightarrow S$, not necessarily smooth, such that $Y \in S m / k$.) Consequently, we could equivalently define a presheaf with $K_{0}$ transfers to be a presheaf with $K_{0}^{\oplus}$ transfers such that for all smooth curves $X \rightarrow S$, the structure map

$$
\theta_{X / S}: K_{0}^{f i n, \oplus}(X / S) \otimes F(X) \rightarrow F(S)
$$

factors through the canonical surjection $K_{0}^{f i n, \oplus}(X / S) \otimes F(X) \rightarrow K_{0}^{f i n}(X / S) \otimes$ $F(X)$.

Recall that a presheaf of abelian groups $F$ defined on $S m / k$ is said to be homotopy invariant if the natural map $F(X) \rightarrow F\left(X \times \mathbb{A}^{1}\right)$ is an isomorphism for all $X$ in $S m / k$.

Proposition 4.4. A presheaf with $K_{0}$ transfers is naturally a presheaf with $K_{0}^{\oplus}$ transfers. Conversely, a homotopy invariant presheaf with $K_{0}^{\oplus}$ transfers is naturally a presheaf with $K_{0}$ transfers.

Proof. The first assertion follows from the naturality of the map $K^{f i n, \oplus}(X / S) \rightarrow$ $K^{f i n}(X / S)$. For a smooth curve $X \rightarrow S$, define $\left[K_{0}^{f i n}(X / S)\right]\left(\operatorname{resp} .,\left[K_{0}^{f i n, \oplus}(X / S)\right]\right)$ to be the cokernel of the map

$$
i_{1}^{*}-i_{0}^{*}: K_{0}^{f i n}\left(X \times \mathbb{A}^{1} / S \times \mathbb{A}^{1}\right) \rightarrow K_{0}^{f i n}(X / S)
$$

(resp., the map obtained by replacing $K_{0}^{f i n}$ with $K_{0}^{f i n, \oplus}$ ), where $i_{\epsilon}: S \rightarrow S \times$ $\mathbb{A}^{1}$ is inclusion at $\epsilon \in \mathbb{A}^{1}$. (Note that $\left[K_{0}^{f i n}(X / S)\right]$ is naturally isomorphic to $K_{\text {geom }}^{0}(X / S)$.) Then the groups $\left[K_{0}^{f i n}(X / S)\right]$ enjoy the same functoriality as do $K_{0}^{f i n}(X / S)$ and the canonical surjection $K_{0}^{f i n}(X / S) \rightarrow\left[K_{0}^{f i n}(X / S)\right]$ is natural in $S$, and similarly for $K_{0}^{f i n, \oplus}(X / S) \rightarrow\left[K_{0}^{f i n, \oplus}(X / S)\right]$. If $F$ is a homotopy invariant presheaf with $K_{0}$ transfers, then the structure maps induce natural pairings

$$
\left[K_{0}^{f i n}(X / S)\right] \otimes F(X) \rightarrow F(S) .
$$

The result will follow once we show the natural surjection $\left[K_{0}^{f i n, \oplus}(X / S)\right] \rightarrow$ $\left[K_{0}^{f i n}(X / S)\right]$ is actually an isomorphism for all smooth curves $X \rightarrow S$. To show this, suppose

$$
0 \rightarrow M^{\prime} \rightarrow M \rightarrow M^{\prime \prime} \rightarrow 0
$$

is a short exact sequence in $\mathcal{P}^{\text {fin }}(X / S)$. Let $N[t] \in \mathcal{P}^{\text {fin }}\left(X \times \mathbb{A}^{1} / S \times \mathbb{A}^{1}\right)$, for $N \in \mathcal{P}^{\text {fin }}(X / S)$, denote the pullback of $N$ along the projection $S \times \mathbb{A}^{1} \rightarrow S$, where 
$\mathbb{A}^{1}=\operatorname{Spec} k[t]$. Then we have an exact sequence in $\mathcal{P}^{\text {fin }}\left(X \times \mathbb{A}^{1} / S \times \mathbb{A}^{1}\right)$ of the form

$$
0 \rightarrow M^{\prime}[t] \rightarrow M[t] \rightarrow M^{\prime \prime}[t] \rightarrow 0
$$

Form the diagram of coherent sheaves on $X \times \mathbb{A}^{1}$

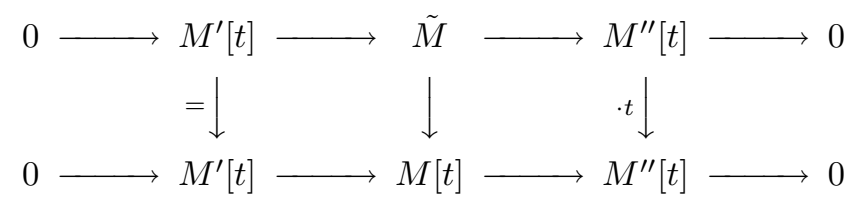

with exact rows and such that the right-hand square is Cartesian. Then $\tilde{M}$ belongs to $\mathcal{P}^{f i n}\left(X \times \mathbb{A}^{1} / S \times \mathbb{A}^{1}\right)$ and satisfies $i_{0}^{*} \tilde{M} \cong M^{\prime} \oplus M^{\prime \prime}$ and $i_{1}^{*} \tilde{M} \cong M$. Since the kernel of $K_{0}^{f i n, \oplus}(X / S) \rightarrow K_{0}^{f i n}(X / S)$ is generated by elements of the form $[M]-\left[M^{\prime}\right]-\left[M^{\prime \prime}\right]$, it follows that $\left[K_{0}^{f i n, \oplus}(X / S)\right] \cong\left[K_{0}^{f i n}(X / S)\right]$.

Recall that for a smooth curve $\pi: X \rightarrow S$, Voevodsky defines $c_{\text {equi }}^{0}(X / S)$ to be the group of relative Cartier divisors (cf. [29, §2]). Equivalently, $c_{\text {equi }}^{0}(X / S)$ is the free abelian group on closed, integral subschemes $Z \subset X$ such that the induced map $Z \rightarrow S$ is finite and dominates a connected component of $S$. (Note that we have not assumed $S$ is connected.) Given a map $f: S^{\prime} \rightarrow S$ of smooth varieties, there is an induced map

$$
f^{*}: c_{\text {equi }}^{0}(X / S) \rightarrow c_{\text {equi }}^{0}\left(X^{\prime} / S^{\prime}\right),
$$

where $X^{\prime}=X \times_{S} S^{\prime}$, given by pullback of cycles as defined, for example, in [22] V.C.7]. Specifically, if $Z \subset X$ is a closed, integral subscheme finite and dominant over a component of $S$, then $f^{*}([Z])$ is given by the formula

$$
f^{*}([Z])=\sum_{w} \operatorname{length}\left(\left(f^{*} \mathcal{O}_{Z}\right)_{w}\right)[\bar{w}]=\sum_{w} \operatorname{length}\left(\mathcal{O}_{Z \times X_{X} X^{\prime}, w}\right)[\bar{w}],
$$

where $w$ ranges over points of $X^{\prime}$ whose closures are finite and dominant over a component of $S^{\prime}$.

A pretheory, as defined by Voevodsky in [29, 3.1], is a presheaf satisfying the hypotheses of Definition 4.1 with $K_{0}^{f i n, \oplus}(-)$ replaced by $c_{\text {equi }}^{0}(-)$ everywhere.

Proposition 4.5. Given a smooth curve $\pi: X \rightarrow S$, there is a well-defined cycle map

$$
\operatorname{cyc}_{X / S}: K_{0}^{f i n}(X / S) \rightarrow c_{e q u i}^{0}(X / S)
$$

given on a generator $[M], M \in \mathcal{P}^{f i n}(X / S)$, by cyc $([M])=\sum_{x} \operatorname{length}\left(M_{z}\right)$, where the sum ranges over points $z$ of $X$ whose closures are finite and dominant over some component of $S$. Moreover, the map $c^{\prime} c_{X / S}$ is natural in the sense that given $S^{\prime} \rightarrow S$ with $S^{\prime} \in S m / k$, the diagram

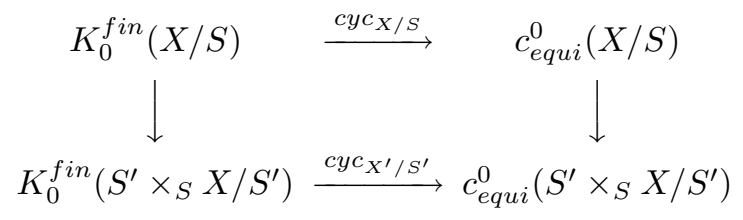

commutes. 
Proof. The function length ${ }_{z}$, for any $z$, and hence the map $c y c_{X / S}$ are additive for short exact sequences in $\mathcal{P}^{f i n}(X / S)$, so that $c y c_{X / S}$ is well defined. The naturality is immediate from the definition of cycle pullback.

Corollary 4.6. Every pretheory is a presheaf with $K_{0}$ transfers and hence a presheaf with $K_{0}^{\oplus}$ transfers.

Suppose $D$ is a generator of $c_{\text {equi }}^{0}(X / S)$. Then the sheaf of ideals $\mathcal{I}_{D}$ cutting out $D$ in $X$ is locally principle. Using the local criterion of flatness [18, 22.6], it follows that the natural map $D \rightarrow S$ is flat, and so the coherent sheaf $\mathcal{O}_{D}$ on $X$ belongs to $\mathcal{P}^{\text {fin }}(X / S)$ and hence determines a class $\left[\mathcal{O}_{D}\right]$ in $K_{0}^{f i n, \oplus}(X / S)$.

Definition 4.7. Given a smooth curve $\pi: X \rightarrow S$, where $X$ and $S$ are regular schemes essentially of finite type over $k$, let

$$
c l_{X / S}: c_{\text {equi }}^{0}(X / S) \rightarrow K_{0}^{f i n, \oplus}(X / S)
$$

be the homomorphism defined on a closed, integral subscheme $Z$ of $X$ which is finite and dominant over $S$ by the formula $c l_{X / S}(Z)=\left[\mathcal{O}_{Z}\right]$. By abuse of notation, we also write $c l$ for the composition of the above map with the canonical surjection $K_{0}^{f i n, \oplus}(X / S) \rightarrow K_{0}^{f i n}(X / S)$.

Note that $c l_{X / S}$ is defined in terms of the standard basis of $c_{\text {equi }}^{0}(X / S)$, and in general $c l_{X / S}(D) \neq\left[\mathcal{O}_{D}\right]$ for an arbitrary Cartier divisor $D$. Additionally, the map $c l_{X / S}$ is not, in general, natural in $S$ (but see the proof of Proposition 4.8 for an important situation in which a form of naturality holds).

Under the cycle map, the element $\left[\mathcal{O}_{Z}\right]$ is sent to $[Z] \in c_{\text {equi }}^{0}(X / S)$, and thus $c l_{X / S}$ gives a (non-natural) splitting of $c y c_{X / S}$. In particular, the map $c y c_{X / S}$ is surjective, and hence a pretheory can equivalently be defined as a presheaf $F$ with $K_{0}^{\oplus}$ transfers such that for all smooth curves $X \rightarrow S$, the structure map $K_{0}^{f i n, \oplus}(X / S) \otimes F(X)$ factors through the natural surjection

$$
K_{0}^{f i n, \oplus}(X / S) \otimes F(X) \stackrel{\text { cyc } \otimes i d}{\longrightarrow} c_{\text {equi }}^{0}(X / S) \otimes F(X) .
$$

Corollary 4.6 admits a partial converse:

Proposition 4.8. Suppose $F$ is a presheaf with $K_{0}$ transfers such that $F$ is separated for the Zariski topology (i.e., the natural map $F(X) \rightarrow \bigoplus_{i} F\left(U_{i}\right)$ is injective for every finite covering $X=\bigcup_{i} U_{i}$ by Zariski open subschemes). Then $F$ admits the structure of a pretheory in a natural manner.

Proof. The map $c l_{X / S}$ is not natural in $S$ in general, but we do have the following fact: If $f: T \rightarrow S$ is any morphism of $k$-schemes, where $T=\operatorname{Spec} \mathcal{O}_{U ; u_{1}, \ldots, u_{m}}$ is the spectrum of the semi-localization of a smooth variety $U$ at a finite set of points $\left\{u_{1}, \ldots, u_{m}\right\}$, and $Y=X \times_{S} T$, then the diagram

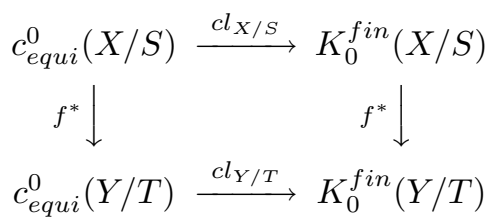

commutes. To see this, it suffices to let $Z \subset X$ be a closed, integral subscheme which is finite and dominant over a component of $S$ and show that $\sum_{w} \operatorname{length}\left(\mathcal{O}_{W, w}\right)\left[\mathcal{O}_{\bar{w}}\right]$ $=\left[\mathcal{O}_{W}\right]$ in $K_{0}^{f i n}(Y / T)$, where $W=Z \times_{X} Y$ and $w$ ranges over the generic points 
of $W$. Since $T$ is the spectrum of a semi-local ring, so is $W$. It follows that $W=\operatorname{Spec} R / f$, where $R$ is the UFD obtained by semi-localizing $Y$ at the closed points of $W$ and $f$ is a non-zero divisor of $R$ such that $\operatorname{Spec} R / f \rightarrow T$ is finite. Moreover, the irreducible components of $W$ are cut out by the terms $p_{i}$ in an irreducible factorization $f=p_{1} \ldots p_{l}$. Using the short exact sequence of objects in $\mathcal{P}^{\text {fin }}(Y / T)$

$$
0 \rightarrow R / p_{1} \stackrel{\cdot p_{2} \ldots p_{l}}{\longrightarrow} R / f \rightarrow R /\left(p_{2} \ldots p_{l}\right) \rightarrow 0
$$

and induction, the desired equation is seen to hold.

Given $F$ as in the statement of the proposition, define pairings

$$
c_{\text {equi }}^{0}(X / S) \otimes F(X) \rightarrow F(S),
$$

for $X \rightarrow S$ a smooth curve in $S m / k$, by taking the composition of

$$
c_{\text {equi }}^{0}(X / S) \otimes F(X) \stackrel{{ }^{l_{X / S} \otimes i d}}{\longrightarrow} K_{0}^{f i n}(X / S) \otimes F(X) \rightarrow F(S) .
$$

The normalization and additivity axioms of a pretheory are readily seen to hold. To establish naturality, suppose we are given a smooth curve $X \rightarrow S$ and a morphism $S^{\prime} \rightarrow S$ in $S m / k$. Since $F$ is Zariski separated, the map $F\left(S^{\prime}\right) \rightarrow \prod_{s^{\prime}} F\left(\operatorname{Spec} \mathcal{O}_{S^{\prime}, s^{\prime}}\right)$ is injective, where $s^{\prime}$ ranges over all closed points of $S^{\prime}$ and $F\left(\operatorname{Spec} \mathcal{O}_{S^{\prime}, s^{\prime}}\right)$ is the stalk of $F$ at $s^{\prime}$ (defined as $\lim _{s^{\prime} \in V \subset S^{\prime}} F(V)$ with $V$ ranging over all Zariski open neighborhoods of $s^{\prime}$ in $V$ ). Fixing such an $s^{\prime} \in S^{\prime}$, set $T=\operatorname{Spec} \mathcal{O}_{S^{\prime}, s^{\prime}}$ and $Y=$ $T \times{ }_{S} X$. To show the commutativity of the left-hand square in

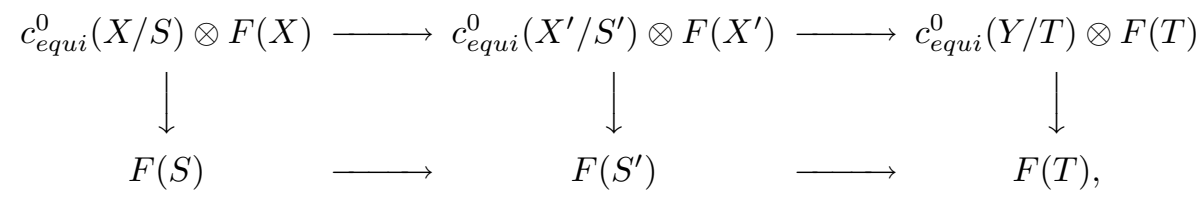

it suffices to show the commutativity of the outer rectangle for all such $T$. But this follows from the commutativity of (6) and the fact that $F$ is a presheaf with $K_{0}$ transfers.

Remark 4.9. Suppose $F$ is a pretheory whose underlying presheaf is Zariski separated. By Corollary 4.6, $F$ has the structure of a presheaf with $K_{0}$ transfers and by Proposition 4.8 is has the induced structure of a pretheory. These two potentially different pretheory structures on the presheaf $F$ coincide, since the composition

$$
c_{\text {equi }}^{0}(X / S) \rightarrow K_{0}^{f i n}(X / S) \rightarrow c_{\text {equi }}^{0}(X / S)
$$

is readily seen to be the identity.

Suppose now that $G$ is a Zariski separated presheaf with $K_{0}$ transfers. Then $G$ admits the structure of a pretheory by Proposition 4.8 and hence the structure of a presheaf with $K_{0}$ transfers by Corollary 4.6. It is unclear whether these potentially different structures of $K_{0}$ transfer map on the presheaf $G$ coincide, since the composition of

$$
K_{0}^{f i n}(X / S) \rightarrow c_{\text {equi }}^{0}(X / S) \rightarrow K_{0}^{f i n}(X / S)
$$

is not known to be the identity map.

Our next goal is to show that a Zariski sheafification of a homotopy invariant presheaf $F$ with $K_{0}$ transfers has the natural structure of a homotopy invariant presheaf with $K_{0}$ transfers. In fact, we will show in Theorem 4.17 below that the Zariski separated presheaf $s(F)$ associated to $F$ is a homotopy invariant presheaf 
with $K_{0}$ transfers such that the natural map $F \rightarrow s(F)$ is a map of presheaves with $K_{0}$ transfers. In light of Proposition 4.8, this suffices to show that the Zariski separated presheaf associated to a homotopy invariant presheaf with $K_{0}$ transfers is in fact a pretheory (see Corollary 4.19 below). Thus, Theorem 4.17 represents the central result in the theory of presheaves with $K_{0}$ transfers, since it allows one to apply all of Voevodsky's results on pretheories to presheaves with $K_{0}$ transfers. However, in order to prove Theorem 4.17, we must first reproduce several of the basic results on pretheories in the context of presheaves with $K_{0}$ transfers.

We will be using the concept of a standard triple as defined in [29, 4.1], except that we find it convenient to add two additional hypotheses. Namely, define a nice standard triple to be a triple $\left(\pi: \bar{X} \rightarrow S, X_{\infty}, Z\right)$ such that $S$ belongs to $S m / k$, the scheme $\bar{X}$ is a normal, quasi-projective $k$-variety, and $Z$ and $X_{\infty}$ are reduced, closed subschemes of $\bar{X}$. Such a triple is required to satisfy the conditions that

(1) the morphism $\pi$ is proper having fibers of dimension one,

(2) $Z \cap X_{\infty}=\emptyset$,

(3) the closed subscheme $X_{\infty} \amalg Z$ of $\bar{X}$ has an open neighborhood $V$ such that $V \rightarrow S$ is an affine morphism and $V$ is the complement of an effective divisor of $\bar{X}$, and

(4) the map $\bar{X}-X_{\infty} \rightarrow S$ is smooth and quasi-affine (i.e., can be factored as an open immersion followed by an affine morphism).

For such a triple, we will customarily write $X$ for $\bar{X}-X_{\infty}$, so that by hypothesis $X \rightarrow S$ is a smooth, quasi-affine curve. The definition of a nice standard triple differs from Voevodsky's definition of a standard triple in that Voevodsky does not require $V$ to be the complement of a divisor or that $\bar{X}$ be quasi-projective. (These modifications turn out to be insignificant, since the proof of Voevodsky's key lemma [29, 4.9], which is sketched in [33 4.3], actually results in the construction of nice standard triples.)

Definition 4.10. Given a nice standard triple $\left(\bar{X} \rightarrow S, X_{\infty}, Z\right)$, define

$$
\mathcal{K}\left(\bar{X} \text { near } X_{\infty}\right)
$$

to be the direct limit of $K$-theory spectra

$$
\underset{W}{\lim _{W}} \mathcal{K}(\bar{X}-W)
$$

where $W$ ranges over all closed subscheme of $\bar{X}$ that are contained in $X$ (equivalently, that miss $X_{\infty}$ ).

Assume now that $S$ is affine. Then the hypotheses of a nice standard triple imply that $X_{\infty}$ is contained in an affine open subscheme $V=\operatorname{Spec} R$ of $\bar{X}$, and so $X_{\infty}=\operatorname{Spec} R / I$ for some ideal $I \subset R$. In this case, we have the identity:

$$
\mathcal{K}\left(\bar{X} \text { near } X_{\infty}\right) \cong \mathcal{K}\left((1+I)^{-1} R\right),
$$

where $(1+I)$ is the multiplicatively closed subset of $R$ consisting of all elements of the form $1+a$ for $a \in I$. To simplify the notation, we define

$$
R_{\langle I\rangle}=(1+I)^{-1} R,
$$

so that $\mathcal{K}\left(\bar{X}\right.$ near $\left.X_{\infty}\right) \cong \mathcal{K}\left(R_{\langle I\rangle}\right)$. 
In the situation of Definition 4.10 the saturation of $1+I$ in $R$ is precisely the set of elements of $R$ which map to units in $R / I$. In particular, the ideal $I \cdot R_{\langle I\rangle}$ is the Jacobson radical of $R_{\langle I\rangle}$ and $R_{\langle I\rangle} / I \cdot R_{\langle I\rangle} \cong R / I$.

Proposition 4.11. Suppose $\left(\pi: \bar{X} \rightarrow S, X_{\infty}, Z\right)$ is a nice standard triple such that $S$ is a smooth, affine $k$-variety and let $X=\bar{X}-X_{\infty}$. Then there is a homotopy fibration sequence of spectra

$$
\mathcal{K}^{\text {fin }}(X / S) \rightarrow \mathcal{K}(\bar{X}) \rightarrow \mathcal{K}\left(\bar{X} \text { near } X_{\infty}\right)
$$

and thus a long exact sequence of $K$-groups of the form

$$
\cdots \rightarrow K_{1}\left(\bar{X} \text { near } X_{\infty}\right) \rightarrow K_{0}^{f i n}(X / S) \rightarrow K_{0}(\bar{X}) \rightarrow K_{0}\left(\bar{X} \text { near } X_{\infty}\right) \rightarrow 0 .
$$

Proof. Assume $\bar{X}$ is embedded in $\mathbb{P}_{k}^{N}$. We claim that for each $W \subset X$ appearing in the direct limit defining $K\left(\bar{X}\right.$ near $\left.X_{\infty}\right)$, there is an effective divisor $D$ in $\bar{X}$ such that $W \subset D \subset X$. To see this, let $\mathcal{L}$ be a line bundle on $\bar{X}$ and $g$ be an element of $\Gamma(\bar{X}, \mathcal{L})$ such that $V=\bar{X}_{g}$ (the locus of points on which $g$ does not vanish) is an open, affine neighborhood of $X_{\infty}$. (The existence of such an $\mathcal{L}$ and $g$ is part of the hypothesis of a nice standard triple.) Say $V=\operatorname{Spec} R$ so that $X_{\infty}=\operatorname{Spec} R / J$ for some ideal $J$. Observe that $W \cap X_{\infty}=\emptyset$ implies that $J+\Gamma\left(V, \mathcal{I}_{W}\right)=R$, where $\mathcal{I}_{W}$ is the sheaf of ideals defining $W$. So there is $h \in \Gamma\left(V, \mathcal{I}_{W}\right) \subset R$ which is congruent to $1 \bmod J$. By [16, II.5.14], we have that $g^{N} f \in \Gamma\left(V, \mathcal{I}_{W} \otimes L\right)$ lifts to an element of $\Gamma\left(\bar{X}, \mathcal{I}_{W} \otimes L\right)$, for $N \gg 0$, which determines a divisor $D$. One may readily verify that $D \cap X_{\infty}=\emptyset$ and $W \subset D$.

Thus in the direct limit defining $K\left(\bar{X}\right.$ near $\left.X_{\infty}\right)$, we may assume each $W=D$ is an effective divisor. For a such a $D$, we have by [11, Theorem on p. 229] that the sequence of exact functors

$$
\mathbb{H}(\bar{X}, D) \rightarrow \mathcal{P}(\bar{X}) \rightarrow \mathcal{P}(\bar{X}-D)
$$

induces a homotopy fibration sequence of associated $K$-theory spectra. Here, $\mathbb{H}(\bar{X}, D)$ is the category consisting of coherent sheaves $M$ on $\bar{X}$ such that $\left.M\right|_{\bar{X}-D}=$ 0 and $M$ admits a resolution of length one by locally free sheaves on $\bar{X}$. Taking limits, the sequence of exact functors

$$
\underset{D}{\lim _{D}} \mathbb{H}(\bar{X}, D) \rightarrow \mathcal{P}(\bar{X}) \rightarrow \underset{D}{\lim } \mathcal{P}(\bar{X}-D)
$$

induces a homotopy fibration sequence on $K$-theory spectra. Note that the singular locus of $\bar{X}$ is contained in $X_{\infty}$, and so $K_{0}(\bar{X}) \rightarrow K_{0}\left(\bar{X}\right.$ near $\left.X_{\infty}\right)$ is surjective (cf. $[38,1.4])$. For each $D$, since $D \subset X$, the category $\mathbb{H}(\bar{X}, D)$ may naturally be regarded as a full subcategory of $\mathcal{M}(X)$. We claim that $\lim _{D} \mathbb{H}(\bar{X}, D)$ coincides with $\mathcal{P}^{\text {fin }}(X / S)$ as subcategories of $\mathcal{M}(X)$, which suffices to finish the proof.

Suppose $M \in \mathbb{H}(\bar{X}, D)$ for some such effective divisor $D$. Then as $D \rightarrow S$ is finite and quasi-affine, it is finite, and so $M$ is finite over $S$. Moreover, by hypothesis there is an exact sequence of coherent sheaves on $X, 0 \rightarrow E_{1} \rightarrow E_{0} \rightarrow M \rightarrow 0$, with $E_{0}$ and $E_{1}$ locally free, and hence an exact sequence

$$
0 \rightarrow \mathcal{T}_{\text {or }}^{\mathcal{O}_{X}}\left(M, \mathcal{O}_{D}\right) \rightarrow E_{1} \otimes_{\mathcal{O}_{X}} \mathcal{O}_{D} \rightarrow E_{0} \otimes_{\mathcal{O}_{X}} \mathcal{O}_{D} \rightarrow M \otimes_{\mathcal{O}_{X}} \mathcal{O}_{D} \rightarrow 0
$$

Since $M$ is supported on $D$, we have that $M \otimes_{\mathcal{O}_{X}} \mathcal{O}_{D} \cong M$ and $\mathcal{T}_{\text {or }} \mathcal{O}_{X}\left(M, \mathcal{O}_{D}\right) \cong$ $M \otimes_{\mathcal{O}_{D}} \mathcal{I} / \mathcal{I}^{2}$, where $\mathcal{I}$ is the sheaf of ideals defining $D$. Since $\mathcal{I} / \mathcal{I}^{2}$ is an invertible sheaf on $D, M$ and $\mathcal{T}_{\text {or }}{ }_{1}^{\mathcal{O}_{X}}\left(M, \mathcal{O}_{D}\right)$ have the same Tor dimension when regarded as sheaves on $D$. Since $\mathcal{O}_{D}$ is a finite, flat $\mathcal{O}_{S}$-module, these modules also have the 
same Tor dimension as $\mathcal{O}_{S}$-modules. Since $X \rightarrow S$ is smooth, $M$ has Tor dimension at most one as an $\mathcal{O}_{S}$-module. But now the sequence (17) shows $\operatorname{Tor}_{1}^{\mathcal{O}_{Y}}\left(M, \mathcal{O}_{D}\right)$, and hence $M$, is $\mathcal{O}_{S}$-flat. We have thus proven $M$ belongs to $\mathcal{P}^{\text {fin }}(X / S)$.

Conversely, suppose $N$ belongs to $\mathcal{P}^{\text {fin }}(X / S)$. As argued above, $N$ is supported on an effective divisor $D$ of $\bar{X}$ which misses $X_{\infty}$. It suffices to show $N$ has Tor dimension one locally on $\bar{X}$ (for then, since $\bar{X}$ is quasi-projective, $N$ admits a resolution of length one by locally free $\mathcal{O}_{\bar{X}}$-modules). Let $x \in X$ be any point in the support of $N$, and let $s$ be its image in $S$. Then there is a formally smooth map of relative dimension one $\mathcal{O}_{S, s} \rightarrow \mathcal{O}_{X, x}$ of local rings, and $N_{x}$ is an $\mathcal{O}_{X, x}$-module which is finite and flat as an $\mathcal{O}_{S, s}$-module. Letting $\mathfrak{m}$ and $\mathfrak{n}$ denote the maximal ideals of $\mathcal{O}_{S, s}$ and $\mathcal{O}_{X, x}$, respectively, we have the spectral sequence

$$
\operatorname{Tor}_{p}^{\mathcal{O}_{X, x} / \mathfrak{m} \cdot \mathcal{O}_{X, x}}\left(\operatorname{Tor}_{q}^{\mathcal{O}_{S, s}}\left(N_{x}, \mathcal{O}_{S, s} / \mathfrak{m}\right), \mathcal{O}_{X, x} / \mathfrak{n}\right) \Longrightarrow \operatorname{Tor}_{p+q}^{\mathcal{O}_{X, x}}\left(N_{x}, \mathcal{O}_{X, x} / \mathfrak{n}\right)
$$

Observe that $\operatorname{Tor}_{q}^{\mathcal{O}_{S, s}}\left(N_{x}, \mathcal{O}_{S, s} / \mathfrak{m}\right)=0$ for $q>0$ since $N_{x}$ is $\mathcal{O}_{S, s}$-flat. On the other hand, $\mathcal{O}_{X, x} / \mathfrak{m} \cdot \mathcal{O}_{X, x}$ is a local ring for a curve over the field $\mathcal{O}_{S, s} / \mathfrak{m}$, and so

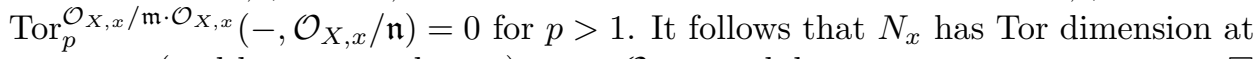
most one (and hence exactly one) as an $\mathcal{O}_{X, x}$-module .

Corollary 4.12. Under the hypothesis of Proposition 4.11, there is a long exact sequence of the form

$$
\begin{gathered}
\cdots \rightarrow K_{1}\left(\bar{X} \text { near } X_{\infty} \amalg Z\right) \rightarrow K_{0}^{f i n}((X-Z) / S) \oplus K_{1}\left(\bar{X} \text { near } X_{\infty}\right) \rightarrow K_{0}^{f i n}(X / S) \\
\rightarrow K_{0}\left(\bar{X} \text { near } X_{\infty} \amalg Z\right) \rightarrow K_{0}\left(\bar{X} \text { near } X_{\infty}\right) \rightarrow 0 .
\end{gathered}
$$

Proof. Observe that $\left(\bar{X} \rightarrow S, X_{\infty} \amalg Z, \emptyset\right)$ is also a nice standard triple with $\bar{X}-$ $\left(X_{\infty} \amalg Z\right)=X-Z$. Moreover, there are natural maps $\mathcal{K}^{f i n}((X-Z) / S) \rightarrow$ $\mathcal{K}^{\text {fin }}(X / S)$ (induced by pushforward along $\left.X-Z \longmapsto X\right)$ and $\mathcal{K}\left(\bar{X}\right.$ near $\left.X_{\infty} \amalg Z\right) \rightarrow$ $\mathcal{K}\left(\bar{X}\right.$ near $\left.X_{\infty}\right)$ (induced by restriction) such that the diagram

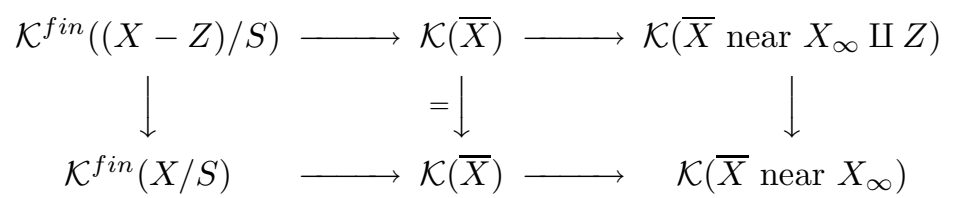

commutes up to homotopy. By Proposition 4.11 the rows of this diagram are homotopy fibration sequences, from which the result follows.

The following theorem is the key technical result concerning presheaves with $K_{0}$ transfers and should be compared with Voevodsky's notion of a "splitting" of a standard triple [29, 4.4]. To set up the statement, recall from the proof of Proposition 4.4 that $\left[K_{0}^{f i n, \oplus}(X / S)\right]=\left[K_{0}^{f i n}(X / S)\right]$ is the cokernel of

$$
K_{0}^{f i n}\left(X \times \mathbb{A}^{1} / S \times \mathbb{A}^{1}\right) \stackrel{i_{1}^{*}-i_{0}^{*}}{\longrightarrow} K_{0}^{f i n}(X / S) .
$$

Given $\alpha \in K_{0}^{f i n}(X / S)$, let $[\alpha]$ denote its image in $\left[K_{0}^{f i n}(X / S)\right]$ under the canonical surjection. Also, given a nice standard triple $\left(\bar{X} \rightarrow S, X_{\infty}, Z\right)$ with $X=\bar{X}-$ $X_{\infty}$, observe that there is a natural map $K_{0}^{f i n}(X / S) \rightarrow K_{0}(\bar{X})$ and hence a map $K_{0}^{f i n}(X / S) \rightarrow K_{0}(Z)$ induced by composition. 
Theorem 4.13. Let $\left(\pi: \bar{X} \rightarrow S, X_{\infty}, Z\right)$ be a nice standard triple such that $S$ is a smooth, affine $k$-variety. If $\alpha$ lies in the kernel of the natural map $K_{0}^{f i n}(X / S) \rightarrow$ $K_{0}(Z)$, then $[\alpha] \in\left[K_{0}^{f i n}(X / S)\right]$ lies in the image of the natural map

$$
\left[K_{0}^{f i n}(X-Z / S)\right] \rightarrow\left[K_{0}^{f i n}(X / S)\right]
$$

Proof. From the hypotheses of a nice standard triple, $X_{\infty} \amalg Z$ is contained in an affine open subscheme $V=\operatorname{Spec} R$ of $\bar{X}$, so that $X_{\infty}=\operatorname{Spec} R / I$ and $Z=\operatorname{Spec} R / J$ with $I+J=R$. Hence $K_{i}\left(\bar{X}\right.$ near $\left.X_{\infty}\right)=K_{i}\left(R_{\langle I\rangle}\right)$ and $K_{i}\left(\bar{X}\right.$ near $\left.X_{\infty} \amalg Z\right)=$ $K_{i}\left(R_{\langle I \cap J\rangle}\right)$. For any ideal $L \subset R$, the Jacobson radical of $R_{\langle L\rangle}$ is $L \cdot R_{\langle L\rangle}$ and we have $R_{\langle L\rangle} / L \cdot R_{\langle L\rangle} \cong R / L$. Thus, using [2, IX.1.3], the natural map

$$
K_{0}\left(R_{\langle L\rangle}\right) \rightarrow K_{0}(R / L)
$$

is an injection and the natural map

$$
K_{1}\left(R_{\langle L\rangle}\right) \rightarrow K_{1}(R / L)
$$

is a surjection with kernel $\left(1+L \cdot R_{\langle L\rangle}\right)^{\times}$. Here, $\left(1+L \cdot R_{\langle L\rangle}\right)^{\times}$is the multiplicative group of units congruent to $1 \bmod L$, which for the sake of brevity we will write as $U(R, L)$. Note that $U(R, L)$ is the subgroup of the multiplicative group of non-zero elements of the field of fractions of $R$ consisting of those elements of the form $\frac{1+a}{1+b}$, where $a, b \in L$.

The injectivity of (8) implies the map

$$
K_{0}\left(\bar{X} \text { near } X_{\infty} \amalg Z\right) \rightarrow K_{0}\left(X_{\infty}\right) \oplus K_{0}(Z)
$$

is injective. Since $K_{0}^{\text {fin }}(X / S) \rightarrow K_{0}\left(X_{\infty}\right)$ is the zero map by Corollary 4.12, we see that the kernel of

$$
K_{0}^{f i n}(X / S) \rightarrow K_{0}\left(\bar{X} \text { near } X_{\infty} \amalg Z\right)
$$

coincides with the kernel of $K_{0}^{f i n}(X / S) \rightarrow K_{0}(Z)$. Thus $\alpha$ lifts to an element of $K_{0}^{f i n}((X-Z) / S) \oplus K_{1}\left(\bar{X}\right.$ near $\left.X_{\infty}\right)$ under the map of Corollary 4.12

Let $\left[K_{1}\left(\bar{X}\right.\right.$ near $\left.\left.X_{\infty}\right)\right]$ denote the cokernel of

$$
i_{1}^{*}-i_{0}^{*}: K_{1}\left(\bar{X} \times \mathbb{A}^{1} \text { near } X_{\infty} \times \mathbb{A}^{1}\right) \rightarrow K_{1}\left(\bar{X} \text { near } X_{\infty}\right)
$$

and similarly for $\left[K_{1}\left(\bar{X}\right.\right.$ near $\left.\left.X_{\infty} \amalg Z\right)\right]$. A diagram chase shows that the theorem holds provided the natural map

$$
\left[K_{1}\left(\bar{X} \text { near } X_{\infty} \amalg Z\right)\right] \rightarrow\left[K_{1}\left(\bar{X} \text { near } X_{\infty}\right)\right]
$$


is surjective. From (9), we have a commutative diagram

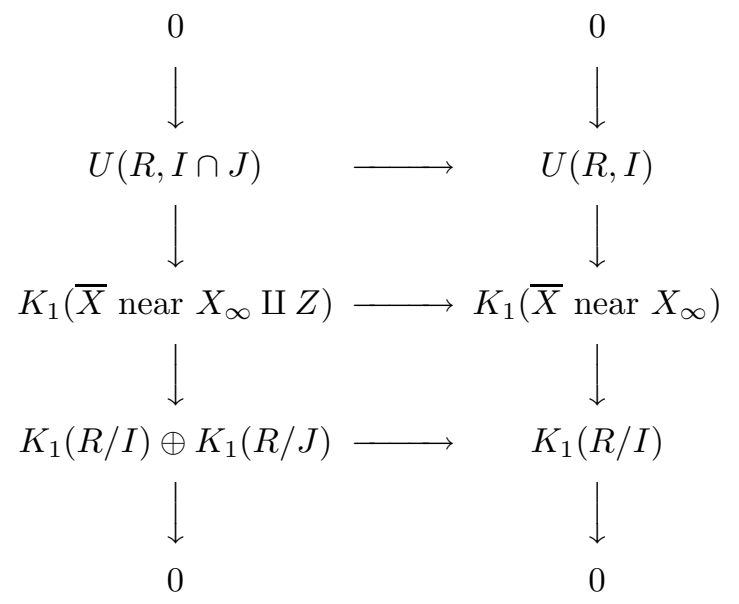

whose columns are exact. Since the bottom arrow of this diagram is surjective, it suffices to show $[U(R, I)]$ is the trivial group, where $[U(R, I)]$ is by definition the cokernel of the map

$$
i_{1}^{*} / i_{0}^{*}: U(R[t], I[t]) \rightarrow U(R, I)
$$

where $i_{\epsilon}^{*}$ is given by evaluation of $t$ at $\epsilon$. (Recall that the $U$ 's are multiplicative groups.) Given $\frac{1+a}{1+b}$ in $U(R, I)$, where $a, b \in I$, the element $\frac{1+t a}{1+t b} \in U(R[t], I[t])$ maps to $\frac{1+a}{1+b}$ under $i_{1}^{*} / i_{0}^{*}$, which implies $[U(R, I)]=1$.

Given a nice standard triple $\left(\bar{X} \rightarrow S, X_{\infty}, Z\right)$ and an open subscheme $j: U \mapsto X$ (where $X=\bar{X}-X_{\infty}$ ), by pulling back along the induced map $U \rightarrow S$, we obtain the triple $\left(U \times_{S} \bar{X}, U \times_{S} X_{\infty}, U \times Z\right)$ which is readily verified to satisfy the hypothesis of a nice standard triple. In this situation, we define $\delta_{U} \in K_{0}^{f i n}\left(U \times_{S} X / U\right)$ to be the class of the structure sheaf of the graph of $(1, j): U \rightarrow U \times_{S} X$. The following theorem is the analogue of a result of Voevodsky concerning pretheories (cf. [29], $4.5])$.

Theorem 4.14. Suppose $\left(\bar{X} \rightarrow S, X_{\infty}, Z\right)$ is a nice standard triple such that $S$ is a smooth, affine $k$-variety. Set $X=\bar{X}-X_{\infty}$ and suppose $i: U \longmapsto X$ is an affine open subscheme. Assume the image of $\delta_{U}$ under the natural map $K_{0}^{f i n}\left(U \times{ }_{S} X / U\right) \rightarrow$ $K_{0}\left(U \times_{S} Z\right)$ is zero. Then for any homotopy invariant presheaf with $K_{0}$ transfers, there is a map $\psi: F(X-Z) \rightarrow F(U)$ such that the diagram

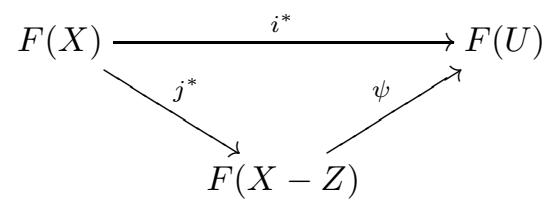

commutes, where $j: X-Z \longmapsto X$ is the canonical embedding.

Proof. By Theorem 4.13 the element $\left[\delta_{U}\right] \in\left[K_{0}^{f i n}\left(U \times_{S} X / U\right)\right]$ lifts to an element $\gamma \in\left[K_{0}^{f i n}\left(U \times{ }_{S}(X-Z) / U\right)\right]$. We define $\psi$ to be the composition of

$$
F(X-Z) \stackrel{\pi^{*}}{\longrightarrow} F\left(U \times_{S}(X-Z)\right) \stackrel{\gamma_{*}}{\longrightarrow} F(U) .
$$


By the naturality axiom of presheaves with $K_{0}$ transfers, the map $\psi \circ j^{*}$ coincides with the composition of

$$
F(X) \stackrel{\pi^{*}}{\longrightarrow} F\left(U \times_{S} X\right) \stackrel{\delta_{*}}{\longrightarrow} F(U) .
$$

The normalization axiom implies that $\delta_{*}=(1, i)^{*}$ and hence $\psi \circ j^{*}=i^{*}$.

The following result is the analogue for presheaves with $K_{0}$ transfers of [29, 4.18].

Corollary 4.15. Let $F$ be a homotopy invariant presheaf with $K_{0}$ transfers, $X$ a connected, quasi-projective smooth $k$-variety, and $x=\left\{x_{1}, \ldots, x_{m}\right\}$ a finite set of closed points on $X$. Write $\mathcal{O}_{X, x}$ for the semi-localization of $X$ at the points of $x$ and let $E$ denote the field of fractions of $X$. Then the natural map

$$
F\left(\operatorname{Spec} \mathcal{O}_{X, x}\right) \rightarrow F(\operatorname{Spec} E)
$$

is an injection (where $F\left(\operatorname{Spec} \mathcal{O}_{X, x}\right)$ and $F(\operatorname{Spec} E)$ are each defined in terms of the evident direct limits).

Proof. It suffices to show that given an open neighborhood $V$ of the finite set $x$ in $X$, an arbitrarily small non-empty open subset $W$ of $X$, and an element $\alpha \in F(V)$ lying in the kernel of $F(V) \rightarrow F(V \cap W)$, there exists a smaller neighborhood $x \subset V^{\prime} \subset V$ such that $\alpha$ vanishes in $F\left(V^{\prime}\right)$. Moreover, we may assume $W \cap x=\emptyset$, and it suffices to prove the result upon replacing $V$ with any smaller open neighborhood of $x$.

Let $Z=V-(V \cap W)$ (the reduced closed complement). Then by [29, 4.9], upon replacing $V$ with a suitably small neighborhood of $x$, we may assume there is a nice standard triple $\left(\pi: \bar{Y} \rightarrow S, Y_{\infty}, Z\right)$, with $S$ smooth and affine, such that $V=Y=\bar{Y}-Y_{\infty}$. (As mentioned above, the proof of [29, 4.9], which is sketched in [33, 4.3], results in the construction of a nice standard triple.) Observe that $\operatorname{Spec} \mathcal{O}_{V, x} \times{ }_{S} Z$ is finite over $\operatorname{Spec} \mathcal{O}_{V, x}$ and is thus the spectrum of a semilocal ring. In particular, $K_{0}\left(\operatorname{Spec} \mathcal{O}_{V, x} \times_{S} Z\right)$ is isomorphic, via the rank map, to the free abelian group on the set of connected components of Spec $\mathcal{O}_{V, x} \times_{S} Z$. It follows that for a sufficiently small neighborhood $V^{\prime}$ of $x$ in $X$, the image of $\delta_{V^{\prime}} \in K_{0}^{f i n}\left(V^{\prime} \times_{S} X / V^{\prime}\right)$ (with $\delta_{V^{\prime}}$ defined as in Theorem 4.14) under the map $K_{0}^{f i n}\left(V^{\prime} \times_{S} X / V^{\prime}\right) \rightarrow K_{0}\left(V^{\prime} \times_{S} X\right)$ is zero. Applying Theorem 4.14 with $X=V$ and $U=V^{\prime}$, we see that the image of $\alpha$ in $F\left(V^{\prime}\right)$ must be zero by the commutativity of the diagram.

Lemma 4.16. Let $F$ be a homotopy invariant presheaf with $K_{0}$ transfers, $X \rightarrow S$ a smooth curve, and $j: U \longmapsto X$ and open embedding. Let $j_{*}: K_{0}^{f i n}(U / S) \rightarrow$ $K_{0}^{f i n}(X / S)$ denote the map induced by the inclusion of categories $\mathcal{P}^{\text {fin }}(U / S) \longmapsto$ $\mathcal{P}^{f i n}(X / S)$. Then given any $\alpha^{\prime} \in K_{0}^{f i n}(U / S)$ and setting $\alpha=j_{*}\left(\alpha^{\prime}\right)$, we have $\alpha_{*}=\alpha_{*}^{\prime} \circ j^{*}$ as maps from $F(U) \rightarrow F(S)$.

Proof. The proof is identical to the proof of [29, 3.12].

Theorem 4.17. Let $F$ be a homotopy invariant presheaf with $K_{0}$ transfers. Then $s(F)$, the Zariski separated presheaf associated to $F$, admits the unique structure of a homotopy invariant presheaf with $K_{0}$ transfers in such a manner that the natural map $F \rightarrow s(F)$ is a morphism of presheaves with $K_{0}$ transfers.

Proof. Observe that the natural map $F \rightarrow s(F)$ is a surjection of presheaves, from which it follows that $s(F)$ is homotopy invariant. Given a smooth curve $X \rightarrow S$ 
and an element $\alpha \in K_{0}^{f i n}(X / S)$, we have a diagram

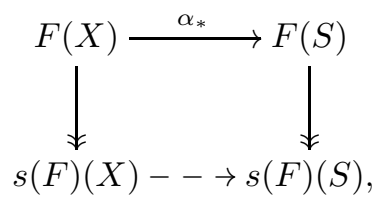

which admits a unique completion provided $\alpha_{*}$ maps the kernel of the left-hand map into the kernel of the right-hand map. Once we prove such a completion exists for all $X \rightarrow S$ and $\alpha$, the axioms of a presheaf with $K_{0}$ transfers are readily verified for $s(F)$.

Suppose $\gamma \in \operatorname{ker}(F(X) \rightarrow s(F)(X))$. Since $s(F)$ is separated, it suffices to prove $\alpha_{*}(\gamma)$ vanishes under the map $F(S) \rightarrow F\left(S_{s}\right)$, where $s$ is an arbitrary closed point of $S$. Note that $\alpha \in K_{0}^{f i n}(X / S)$ is supported on a closed subscheme $Z \subset X$ which is finite over $S$, in the sense that $\alpha$ lies in the image of $K_{0}^{f i n}(Z / S) \rightarrow K_{0}^{f i n}(X / S)$. Let $z$ be the finite set of points of $Z$ lying over $s \in S$. It follows from Corollary 4.15 that $\gamma$ vanishes in a suitably small open neighborhood $U$ of $z$ in $X$. Shrinking $S$ as we may, we may assume $Z \subset U \subset X$, and so $\alpha=j_{*}\left(\alpha^{\prime}\right)$ for some $\alpha^{\prime} \in K_{0}^{f i n}(U / S)$, where $j: U \longmapsto X$ is the inclusion map. From Lemma 4.16 we have that $\alpha_{*}(\gamma)=$ $\alpha_{*}^{\prime}\left(j^{*}(\gamma)\right)=0$

Corollary 4.18. The Zariski separated presheaf $s(F)$ associated to a homotopy invariant presheaf with $K_{0}$ transfers $F$ admits a unique structure of a pretheory so that $F \rightarrow s(F)$ is a morphism of presheaves with $K_{0}$ transfers.

Proof. This follows immediately from the theorem and Proposition 4.8.

Corollary 4.19. The Zariski sheafification $F_{Z a r}$ of a homotopy invariant presheaf with $K_{0}$ transfers $F$ admits a unique structure of a pretheory so that $F \rightarrow F_{Z a r}$ is a morphism of presheaves with $K_{0}$ transfers.

Proof. The follows from Corollary 4.18 and [29, 4.25].

Corollary 4.20. Suppose $F$ is a homotopy invariant presheaf with $K_{0}$ transfers such that $n \cdot F=0$ for some integer $n$ relatively prime to the exponential characteristic of the ground field $k$. Let $\mathcal{O}_{X, x}^{\text {hen }}$ denote the henselization of a smooth $k$-variety $X$ at a closed point $x$. Then the natural map

$$
F\left(\operatorname{Spec} \mathcal{O}_{X, x}^{h e n}\right) \rightarrow F(\operatorname{Spec} k(x))
$$

is an isomorphism.

Proof. This follows by applying [29, 5.25] to the Zariski sheafification of $F$, which is a pretheory by Corollary 4.19

A common method of producing homotopy invariant presheaves with $K_{0}$ transfers from presheaves with $K_{0}^{\oplus}$ transfers is given in the following proposition.

Proposition 4.21. Let $F$ be a presheaf with $K_{0}^{\oplus}$ transfers. Then the homology presheaves of the complex $F\left(-\times \Delta^{\bullet}\right)$ are homotopy-invariant presheaves with $K_{0}$ transfers. 
Proof. For any $X$, the presheaf $F(-\times X)$ is a presheaf with $K_{0}^{\oplus}$ transfers, so that $F\left(-\times \Delta^{\bullet}\right)$ is a complex of presheaves with $K_{0}^{\oplus}$ transfers. Moreover, the kernel and cokernel of a map of presheaves with $K_{0}^{\oplus}$ transfers is again a presheaf with $K_{0}^{\oplus}$ transfers. This proves the homology presheaves of $F\left(-\times \Delta^{\bullet}\right)$ have $K_{0}^{\oplus}$ transfers. A standard argument shows these homology presheaves are homotopy invariant, and so the result follows from Proposition 4.4.

The following theorem and accompanying corollary describe the key properties, at least for the purposes of this paper, exhibited by presheaves with $K_{0}^{\oplus}$ transfers.

Theorem 4.22 (cf. [9, 5.5] and [24, 7.6]). Let $F$ be a presheaf of abelian groups, simplicial abelian groups, group-like $H$-spaces, or connective spectra on $S c h / k$. Assume, for all $q \geq 0$, the restriction of the presheaf of abelian groups $X \mapsto \pi_{q} F(X)$ to $S m / k$ has the structure of a presheaf with $K_{0}^{\oplus}$ transfers. (In case $F$ is a presheaf of abelian groups, we interpret this hypothesis as meaning $F$ is a presheaf with $K_{0}^{\oplus}$ transfers.)

(1) If $k$ is an arbitrary field and $\pi_{q} F(-)_{(S m / k)_{N i s}}$ (the sheafification in the Nisnevich topology of of the restriction of the presheaf $\pi_{q} F(-)$ to the category $S m / k)$ is trivial, then the chain complex $F\left(-\times \Delta^{\bullet}\right)$ is locally acyclic for the Zariski topology on $\mathrm{Sm} / \mathrm{k}$ (i.e., the sheaf homology groups of the chain complex of Zariski sheaves $F\left(-\times \Delta^{d}\right) \tilde{Z}_{a r}$ are all trivial). In particular, we have

$$
\pi_{q}\left|d \mapsto F\left(\Delta^{d}\right)\right|=0,
$$

for all $q \geq 0$.

(2) If $k$ admits resolutions of singularities and $\pi_{q} F(-)_{c d h}^{\sim}$ (the sheafification of the presheaf $\pi_{q} F(-)$ in the cdh topology) is trivial, then the chain complex $F\left(-\times \Delta^{\bullet}\right)$ is locally acyclic for the Zariski topology on $S m / k$. In particular, we have

$$
\pi_{q}\left|d \mapsto F\left(\Delta^{d}\right)\right|=0,
$$

for all $q \geq 0$.

(3) If $k$ is algebraically closed, $n \geq 1$ is an integer relatively prime to the exponential characteristic of $k$, and $\pi_{q} F(-)_{h}^{\sim}$ (the sheafification of the presheaf $\pi_{q} F(-)$ in the $h$ topology) is trivial, then

$$
\pi_{q}\left(\left|d \mapsto F\left(\Delta^{d}\right)\right|, \mathbb{Z} / n\right)=0,
$$

for all $q \geq 0$.

Proof. Using the convergent spectral sequence (cf. [5, B.5])

$$
\pi_{p}\left(d \mapsto \pi_{q}\left(F\left(\Delta^{d}\right) ; A\right)\right) \Longrightarrow \pi_{p+q}\left(\left|d \mapsto F\left(\Delta^{d}\right)\right| ; A\right),
$$

where $A$ is $\mathbb{Z} / n$ or $\mathbb{Z}$, it suffices (by considering $X \mapsto \pi_{q} F(X)$, for all $q \geq 0$ ) to prove each result in the case where $F$ is a presheaf of abelian groups.

The proofs of the first and second cases are identical to the proofs of [29, 5.9] and [9,5.5], respectively, observing that each of these cited proofs only requires that the Zariski sheafification of $h_{i}\left(F\left(-\times \Delta^{\bullet}\right)\right)$ be a pretheory, for all $i \geq 0$. This property holds in our context by Corollary 4.19

To prove the third case, we assume $F_{h}^{\sim}=0$ and we must prove the chain complex $F\left(\Delta^{\bullet}\right) \otimes \mathbb{L} \mathbb{Z} / n$ is acyclic. In fact, it suffices to show $\operatorname{Ext}_{A b}^{*}\left(F\left(\Delta^{\bullet}\right), \mathbb{Z} / n\right)=0$. By Corollary 4.20 we have

$$
\operatorname{Ext}_{A b}^{*}\left(F\left(\Delta^{\bullet}\right), \mathbb{Z} / n\right) \cong \operatorname{Ext}_{e t}^{*}\left(F\left(-\times \Delta^{\bullet}\right)_{e t}^{\sim}, \mathbb{Z} / n\right),
$$


and thus by using [24 7.1 and 10.10], we have the string of isomorphisms

$$
\operatorname{Ext}_{e t}^{*}\left(F\left(-\times \Delta^{\bullet}\right)_{e t}^{\sim}, \mathbb{Z} / n\right) \cong \operatorname{Ext}_{e t}^{*}\left(F_{e t}, \mathbb{Z} / n\right) \cong \operatorname{Ext}_{h}^{*}\left(F_{h}, \mathbb{Z} / n\right)=0 .
$$

Corollary 4.23. Let $\psi: F \rightarrow G$ be a natural transformation of presheaves of abelian groups, simplicial abelian groups, group-like $H$-spaces, or connective spectra on $S c h / k$. Assume that for all $q \geq 0$ the restriction to $S m / k$ of the natural transformation of presheaves of abelian groups

$$
\left(X \mapsto \pi_{q} F(X)\right) \rightarrow\left(X \mapsto \pi_{q} G(X)\right)
$$

is a morphism of presheaves with $K_{0}^{\oplus}$ transfers.

(1) If $k$ is an arbitrary field and $\pi_{q} F(-)_{(S m / k)_{N i s}}^{\sim} \longrightarrow \pi_{q} G(-)_{(S m / k)_{N i s}}$ is an isomorphism, then the natural map

$$
F\left(-\times \Delta^{\bullet}\right) \rightarrow G\left(-\times \Delta^{\bullet}\right)
$$

is locally a weak homotopy equivalence for the Zariski topology on $\mathrm{Sm} / \mathrm{k}$. In particular, the natural map

$$
\pi_{q}\left|d \mapsto F\left(\Delta^{d}\right)\right| \rightarrow \pi_{q}\left|d \mapsto G\left(\Delta^{d}\right)\right|
$$

is an isomorphism, for all $q \geq 0$.

(2) If $k$ admits resolutions of singularities and $\pi_{q} F(-)_{c d h}^{\sim} \rightarrow \pi_{q} G(-)_{c d h}^{\sim}$ is an isomorphism, then the natural map

$$
F\left(-\times \Delta^{\bullet}\right) \rightarrow G\left(-\times \Delta^{\bullet}\right)
$$

is locally a weak homotopy equivalence for the Zariski topology on $S m / k$. In particular, the natural map

$$
\pi_{q}\left|d \mapsto F\left(\Delta^{d}\right)\right| \rightarrow \pi_{q}\left|d \mapsto G\left(\Delta^{d}\right)\right|
$$

is an isomorphism, for all $q \geq 0$.

(3) If $k$ is algebraically closed, $n \geq 1$ is an integer relatively prime to the exponential characteristic of $k$, and $\pi_{q} F(-)_{h} \sim \pi_{q} G(-)_{h}$ is an isomorphism, then the natural map

$$
\pi_{q}\left(\left|d \mapsto F\left(\Delta^{d}\right)\right|, \mathbb{Z} / n\right) \rightarrow \pi_{q}\left(\left|d \mapsto G\left(\Delta^{d}\right)\right|, \mathbb{Z} / n\right)
$$

is an isomorphism, for all $q \geq 0$.

Proof. There is a map of convergent spectral sequences from

$$
\pi_{p}\left(d \mapsto \pi_{q}\left(F\left(\Delta^{d}\right) ; A\right)\right) \Longrightarrow \pi_{p+q}\left(\left|d \mapsto F\left(\Delta^{d}\right)\right| ; A\right)
$$

to

$$
\pi_{p}\left(d \mapsto \pi_{q}\left(G\left(\Delta^{d}\right) ; A\right)\right) \Longrightarrow \pi_{p+q}\left(\left|d \mapsto G\left(\Delta^{d}\right)\right| ; A\right),
$$

where $A$ is $\mathbb{Z} / n$ or $\mathbb{Z}$. As in the proof of the theorem, this allows us to consider only the case where $F$ and $G$ are presheaves of abelian groups. In this case, the result follows by applying the theorem to the cone of the morphism $F \rightarrow G$ (which may be interpreted as a presheaf of simplicial abelian groups).

The following theorem gives most of the instances in which presheaves with $K_{0}$ transfers arise in the remainder of this paper. 
Theorem 4.24. Let $Y$ be a k-scheme, $U$ a locally closed subscheme of $Y, A$ an abelian group, and $q$ an integer. The presheaves

$$
K_{q}^{q f}(-,(U, Y) ; A), \quad K_{q}^{q f}(-, Y ; A), \quad \text { and } \quad K_{q}^{f i n}(-, Y ; A)
$$

are presheaves with $K_{0}$ transfers. The requisite structure maps for $K_{q}^{q f}(-,(U, Y) ; A)$ are induced by the pairings

$$
\mathcal{P}^{q f}(X,(U, Y)) \times \mathcal{P}^{f i n}(X / S) \rightarrow \mathcal{P}^{q f}(S,(U, Y))
$$

sending $(M, N)$ to $\left(\pi \times i d_{U}\right)_{*}\left(M \otimes_{\mathcal{O}_{X \times U}} p^{*} N\right)$, where $\pi: X \rightarrow S$ is any smooth curve and $p: X \times U \rightarrow X$ is the canonical projection, and similarly for the other two presheaves. Likewise,

$$
K_{g q f}^{q}(-,(U, Y) ; A), \quad K_{g q f}^{q}(-, Y ; A), \quad \text { and } \quad K_{g e o m}^{q}(-, Y ; A)
$$

are presheaves with $K_{0}$ transfers.

Moreover, the natural maps $K_{q}^{q f}(-,(U, Y) ; A) \rightarrow K_{q}^{q f}(-, U ; A), K_{q}^{f i n}(-, U ; A)$ $\rightarrow K_{q}^{q f}(-, U ; A)$, and $g_{*}: K_{q}^{f i n}(-, Y ; A) \rightarrow K_{q}^{f i n}\left(-, Y^{\prime} ; A\right)$, where $g: Y \rightarrow Y^{\prime}$ is any morphism, as well as the natural maps $K_{g q f}^{q}(-,(U, Y) ; A) \rightarrow K_{g q f}^{q}(-, U ; A)$, $K_{\text {gqf }}^{q}(-, U ; A) \rightarrow K_{\text {geom }}^{q}(-, U ; A)$, and $g_{*}: K_{\text {geom }}^{q}(-, Y ; A) \rightarrow K_{\text {geom }}^{q}\left(-, Y^{\prime} ; A\right)$ are all morphism of presheaves with $K_{0}$ transfers.

Proof. Given $M \in \mathcal{P}^{q f}(X,(U, Y))$ and $N \in \mathcal{P}^{f i n}(X / S)$, the support of $M \otimes_{\mathcal{O}_{X \times U}}$ $p^{*} N$ in $X \times U$ is finite over $S \times U$. Thus, the functor described in the statement is biexact, thereby inducing a pairing on homotopy groups of associated spectra of the form

$$
K_{q}^{q f}(X,(U, Y) ; A) \otimes K_{0}^{f i n}(X / S) \rightarrow K_{q}^{q f}(S,(U, Y) ; A) .
$$

The axioms of a presheaf with $K_{0}$ transfers are readily verified.

The presheaf $K_{q}^{q f}(-, Y ; A)$ coincides with $K_{q}^{q f}(-,(Y, Y) ; A)$. The assertion for the presheaf $K_{q}^{f i n}(-, Y ; A)$ is proven in exactly the same manner, using $\mathcal{P}^{f i n}$ and $K^{f i n}$ in place of $\mathcal{P}^{q f}$ and $K^{q f}$.

Using strictly functorial models for the exact categories involved as in Remark 3.2, the pairing

$$
\mathcal{K}^{q f}(X,(U, Y)) \wedge \mathcal{K}^{f i n}(X / S) \rightarrow \mathcal{K}^{q f}(S,(U, Y))
$$

is strictly natural on the category $S c h / S$. In particular, we have a pairing of simplicial spectra which at degree $d$ is given as

$$
\mathcal{K}^{q f}\left(X \times \Delta^{d},(U, Y)\right) \wedge \mathcal{K}^{f i n}\left(X \times \Delta^{d} / S \times \Delta^{d}\right) \rightarrow \mathcal{K}^{q f}\left(S \times \Delta^{d},(U, Y)\right) .
$$

This gives the pairing

$$
K_{g q f}^{q}(X,(U, Y) ; A) \otimes K_{g e o m}^{0}(X / S) \rightarrow K_{g q f}^{q}(S,(U, Y) ; A),
$$

and the axioms of a presheaf with $K_{0}$ transfers are readily verified. (Note that $K_{\text {geom }}^{0}(X / S)$ is naturally a quotient of $K_{0}^{f i n}(X / S)$.) The result for $K_{\text {geom }}^{q}(-, Y ; A)$ is proven in exactly the same way.

The final assertion is readily verified. 


\section{LOCALIZATION FOR $K$-HOMOLOGY}

The goal of this section is to prove $\mathcal{K}^{B M}(-)$ admits a "localization" long exact sequence, analogous to that of $G$-theory. Specifically, we prove that the push forward along a closed immersion $Z \longmapsto X$ and the pullback along the open complement $X-Z \longmapsto X$ fit into the expected homotopy fibration sequence. Our proof technique requires us to assume $Z$ and $X$ are Cohen-Macaulay and that either $k$ admits resolutions of singularities or $k$ is algebraically closed, and we use spectra with finite coefficients $\mathcal{K}^{B M}(-; \mathbb{Z} / n)$ for some $n \geq 1$ relatively prime to the exponential characteristic of $k$. We know of no obstruction to the more general result involving a closed immersion of arbitrary varieties defined over an arbitrary base field. However, the result proven here is sufficient for the purposes of this paper.

Lemma 5.1. Let $f: Y \rightarrow X$ be a morphism of quasi-projective varieties, $i: W \subset Y$ a closed subvariety, and $j: U \longmapsto Y$ the open complement of $W$ in $Y$. Then the sequence of exact functors

$$
\mathcal{M}^{q f}(W / X) \stackrel{i_{*}}{\longrightarrow} \mathcal{M}^{q f}(Y / X) \stackrel{j^{*}}{\longrightarrow} \mathcal{M}^{q f}((U, Y) / X)
$$

induces a homotopy fibration sequence of spectra

$$
\mathcal{G}^{q f}(W / X) \stackrel{i_{*}}{\longrightarrow} \mathcal{G}^{q f}(Y / X) \stackrel{j^{*}}{\longrightarrow} \mathcal{G}^{q f}((U, Y) / X)
$$

More generally, suppose $Y \subset \bar{Y}$ is an open embedding and $f$ is the restriction of a morphism $\bar{Y} \rightarrow X$. Then the natural maps

$$
\mathcal{G}^{q f}((W, \bar{Y}) / X) \rightarrow \mathcal{G}^{q f}((Y, \bar{Y}) / X) \rightarrow \mathcal{G}^{q f}((U, \bar{Y}) / X)
$$

form a homotopy fibration sequence.

Proof. The first assertion follows from the second by taking $Y=\bar{Y}$.

The abelian categories $\mathcal{M}^{q f}((W, \bar{Y}) / X), \mathcal{M}^{q f}((Y, \bar{Y}) / X)$, and $\mathcal{M}^{q f}((U, \bar{Y}) / X)$ are given by the filtered direct limits of abelian categories

$$
\underset{Z}{\lim } \mathcal{M}(Z \cap W), \quad \underset{Z}{\lim } \mathcal{M}(Z \cap Y), \quad \text { and } \quad \underset{Z}{\lim } \mathcal{M}(Z \cap U),
$$

where $Z$ ranges over all closed subschemes of $\bar{Y}$ which are quasi-finite over $X$. For each fixed $Z$, the natural maps

$$
\mathcal{G}(Z \cap W) \rightarrow \mathcal{G}(Z) \rightarrow \mathcal{G}(Z \cap U)
$$

form a homotopy fibration sequence of spectra by Quillen's Localization Theorem [20, Theorem 3]. Since a filtered direct limit of homotopy fibration sequences is again a homotopy fibration sequence, the result is proven.

The following proposition allows one (in certain contexts) to replace $\mathcal{K}_{g q f}(U / X)$ with $\mathcal{K}_{g q f}((U, Y) / X)$, where $U \longmapsto Y$ is a locally closed embedding (see Corollary 5.3 below). This result will be use to prove the localization sequence we seek (see Theorem 5.5 below).

Proposition 5.2. Suppose $Y \rightarrow X$ is a flat morphism of quasi-projective $k$ varieties and let $U \subset Y$ be a locally closed subvariety. Let

$$
K_{m}^{q f}(U / X)(-) \quad \text { and } K_{m}^{q f}((U, Y) / X)(-)
$$

denote the presheaves of abelian groups on $S c h / X$ given by the formulas

$$
(V \rightarrow X) \mapsto K_{m}^{q f}\left(U \times_{X} V / V\right) \quad \text { and } \quad(V \rightarrow X) \mapsto K_{m}^{q f}\left(\left(U \times_{X} V, Y \times_{X} V\right) / V\right)
$$


Then the natural map of presheaves

$$
K_{m}^{q f}((U, Y) / X)(-) \rightarrow K_{m}^{q f}(U / X)(-)
$$

becomes an isomorphism upon sheafification in the cdh-topology.

Proof. It suffices to show that given any morphism $V \rightarrow X$ in $S c h / k$ and an element $\alpha$ in the kernel or the cokernel of

$$
K_{m}^{q f}((U, Y) / X)(V) \rightarrow K_{m}^{q f}(U / X)(V),
$$

there is a $c d h$ covering $\left\{p_{i}: V_{i} \rightarrow V\right\}$ of $V$ such that $p_{i}^{*}(\alpha)=0$ for all $i$. Note that if $\operatorname{dim}(V)=0$, the above map is an isomorphism. Proceeding by induction on $\operatorname{dim}(V)$, we claim that it suffices to construct a blow-up $\pi: W \rightarrow V$ with center $C$ of dimension strictly smaller than $V$ such that $\pi^{*}(\alpha)=0$. For if such a blow-up exists, then, by the induction hypothesis, there is a $c d h$ covering $\left\{q_{i}: C_{i} \rightarrow C\right\}$ of $C$ such that $q_{i}^{*}\left(\left.\alpha\right|_{C}\right)=0$, for all $i$, and the collection $\left\{C_{i} \rightarrow C \rightarrow V\right\} \cup\{W \rightarrow V\}$ forms a $c d h$ covering of $V$ with the desired property.

For brevity, write $U_{V}$ for $U \times_{X} V$ and $Y_{V}$ for $Y \times_{X} V$. We have

$$
\mathcal{P}^{q f}\left(U_{V} / V\right)=\underset{Z}{\lim _{Z}} \mathcal{P}^{q f}(Z / V),
$$

where the limit ranges over closed subschemes $Z \subset U_{V}$ which are quasi-finite over $V$. Similarly, $\mathcal{P}^{q f}\left(\left(U_{V}, Y_{V}\right) / V\right)$ is given by the subdirect limit indexed by those $Z$ 's satisfying the additional condition that the closure of $Z$ in $Y_{V}$ is quasi-finite over $V$.

To prove the proposition, it suffices to show that for each closed subscheme $Z \subset U_{V}$ which is quasi-finite and dominant over $V$, there is a blow-up $\pi: W \rightarrow V$ so that the composition of

$$
\mathcal{P}^{q f}(Z / V) \stackrel{\subset}{\longrightarrow} \mathcal{P}^{q f}\left(U_{V} / V\right) \stackrel{\pi^{*}}{\longrightarrow} \mathcal{P}^{q f}\left(U_{W} / W\right)
$$

lands in $\mathcal{P}^{q f}\left(\left(U_{W}, Y_{W}\right) / W\right)$. To see that this suffices, if $\beta \in K_{m}^{q f}\left(U_{V} / V\right)$, then $\beta$ is in the image of $K_{m}^{q f}(Z / V) \rightarrow K_{m}^{q f}\left(U_{V} / V\right)$ for some such $Z$. But then clearly $\pi^{*}(\beta)$ lies in the image of $K_{m}^{q f}\left(\left(U_{W}, Y_{W}\right) / W\right) \rightarrow K_{m}^{q f}\left(U_{W} / W\right)$ as desired. Similarly, if $\alpha$ lies in the kernel of $K_{m}^{q f}\left(\left(U_{V}, Y_{V}\right) / V\right) \rightarrow K_{m}^{q f}\left(U_{V} / V\right)$, then $\alpha$ lies in the image of $K_{m}^{q f}\left(Z_{1} / V\right) \rightarrow K_{m}^{q f}\left(U_{V} / V\right)$ for some $Z_{1} \subset U_{V}$ whose closure in $Y_{V}$ is quasifinite over $V$, and there is a larger closed subscheme $Z_{1} \subset Z \subset U_{V}$, with $Z \rightarrow V$ quasi-finite, such that $\alpha$ belongs to the kernel of the natural map $K_{m}^{q f}\left(Z_{1} / V\right) \rightarrow$ $K_{m}^{q f}(Z / V)$. But then clearly $\pi^{*}(\alpha)=0$ in $K_{m}^{q f}\left(\left(U_{W}, Y_{W}\right) / W\right)$.

To construct such a blow-up $\pi$, let $\bar{Z} \subset Y_{V}$ denote the scheme-theoretic closure of $Z$ in $Y_{V}$. By the platification par eclatement theorem [21], there is a blow-up $\pi: W \rightarrow V$ such that the strict transform $\bar{Z}^{\prime} \rightarrow W$ of $\bar{Z} \rightarrow V$ is flat and hence quasi-finite. Set $Z^{\prime}=\bar{Z}^{\prime} \cap U_{W}$, so that $Z^{\prime} \rightarrow W$ is the strict transform of $Z \rightarrow V$. Since, by hypothesis, $Y_{V} \rightarrow V$ and $U_{V} \rightarrow V$ are flat, their strict transforms along $W \rightarrow V$ coincide with the pullbacks $Y_{W} \rightarrow W$ and $U_{W} \rightarrow W$. Similarly, given $M \in \mathcal{P}^{q f}(Z / V) \subset \mathcal{P}^{q f}\left(U_{V}, V\right)$, since $M$ is $\mathcal{O}_{V}$-flat, its strict transform along $W \rightarrow V$ coincides with the pullback $\pi^{*} M$ as coherent sheaves on $U_{W}$. Moreover, the support of $\pi^{*} M$ is a subscheme of $Z^{\prime}$, since the strict transform of a coherent sheaf supported on $Z$ is supported on $Z^{\prime}$. In particular, the closure of the support of $\pi^{*} M$ in $Y_{W}$ is contained in $\bar{Z}^{\prime}$, which is quasi-finite over $W$. Thus the functor $\pi^{*}: \mathcal{P}^{q f}(Z / V) \rightarrow \mathcal{P}^{q f}\left(U_{W} / W\right)$ lands $\mathcal{P}^{q f}\left(\left(U_{W}, Y_{W}\right) / W\right)$, as desired. 
Corollary 5.3. Let $Y \rightarrow X$ be a morphism of quasi-projective $k$-varieties and let $U \subset Y$ be a locally closed subvariety.

(1) If $k$ is an algebraically closed field and $n$ is an integer relatively prime to the exponential characteristic of $k$, then the natural map

$$
\mathcal{K}_{g q f}(\operatorname{Spec} k,(U, Y)) \rightarrow \mathcal{K}_{g q f}(\operatorname{Spec} k, U)=\mathcal{K}^{B M}(U)
$$

induces an isomorphism

$$
K_{g q f}^{-q}(\operatorname{Spec} k,(U, Y) ; \mathbb{Z} / n) \cong K_{q}^{B M}(U ; \mathbb{Z} / n),
$$

for all $q \geq 0$.

(2) If $k$ is any field admitting resolutions of singularities (for example, if $k$ is any field of characteristic zero), then the natural map

$$
\mathcal{K}_{g q f}(\operatorname{Spec} k,(U, Y)) \rightarrow \mathcal{K}_{g q f}(\operatorname{Spec} k, U)=\mathcal{K}^{B M}(U)
$$

is a weak homotopy equivalence.

Proof. This follows immediately from Proposition 5.2 (for $X=\operatorname{Spec} k$ ), Theorem 4.24, and Corollary 4.23

Recall from Definition 2.2 the simplified bivariant notations $\mathcal{G}^{q f}(X, Y), \mathcal{K}^{q f}(X, Y)$, etc., which stand for, respectively, $\mathcal{G}^{q f}(X \times Y / X), \mathcal{K}^{q f}(X \times Y / X)$, etc.

Proposition 5.4. Let $Y$ be a projective variety, let $U \subset Y$ be a locally closed subvariety, and assume $U$ is Cohen-Macaulay. If $X$ is a smooth, quasi-projective variety, then the natural map

$$
\left.\mathcal{K}^{q f}(X,(U, Y))\right) \rightarrow \mathcal{G}^{q f}(X,(U, Y))
$$

is a weak equivalence of spectra.

Proof. Replacing $Y$ with the closure of $U$, we may assume $U$ is an open, dense subscheme of $Y$. Additionally, we may assume $U$ is connected and hence equidimensional, so that $Y$ is as well.

As the proof of [34, 2.2], Quillen's Resolution Lemma 20, Theorem 3] applies to the inclusion of categories $\mathcal{P}^{q f}(X,(U, Y)) \subset \mathcal{M}^{q f}(X,(U, Y))$, provided that given $M$ in the larger category, there is surjection $E \rightarrow M$ with $E$ in the smaller category. Given such an $M$, assume $M$ is supported on $Z \subset X \times U$ such that the closure $\bar{Z}$ of $Z$ in $X \times Y$ is quasi-finite, and hence finite, over $X$.

Recall $Y$ has been assumed equi-dimensional, say of dimension $d$, and so there exists a finite morphism $\pi: Y \rightarrow \mathbb{P}^{d}$. Since $U$ is Cohen-Macaulay, the map $\left.\pi\right|_{U}$ : $U \rightarrow \mathbb{P}^{d}$ is necessarily flat by $[18,23.1]$. Let $W=\left(1_{X} \times \pi\right)(\bar{Z})$ (the scheme-theoretic image), a closed subscheme of $X \times \mathbb{P}^{d}$ finite over $X$. As shown in the proof of 34 . $2.3]$, there exists a larger closed subscheme $W^{\prime} \subset X \times \mathbb{P}^{d}$ with $W \subset W^{\prime}$ such that $W^{\prime} \rightarrow X$ is finite and flat. Now let $\bar{Z}^{\prime}=\left(1_{X} \times \pi\right)^{-1}\left(W^{\prime}\right)=W^{\prime} \times_{\mathbb{P}^{d}} Y$ and observe that $\bar{Z}$ is contained in $\bar{Z}^{\prime}$ and the morphism $\bar{Z}^{\prime} \rightarrow X$ is finite, since it is the composition $\bar{Z}^{\prime} \rightarrow W^{\prime} \rightarrow X$ of finite morphisms. Set $Z^{\prime}=\bar{Z}^{\prime} \cap X \times U=W^{\prime} \times_{\mathbb{P}^{d}} U$, so that we have the containment $Z \subset Z^{\prime}$ and so that the morphism $Z^{\prime} \rightarrow X$ is quasi-finite. The morphism $Z^{\prime} \rightarrow X$ is flat as well, since it is the composition $Z^{\prime} \rightarrow W^{\prime} \rightarrow X$ of flat morphism. Regarding $M$ as a coherent $\mathcal{O}_{Z^{\prime}}$-module as we may, we can find a locally free $\mathcal{O}_{Z^{\prime}}$-module $E$ and a surjection $E \rightarrow M$. Since $E \in \mathcal{P}^{q f}(X,(U, Y))$, the result follows. 
In the following theorem, we write $\mathcal{K}^{B M}(Y ; \mathbb{Z} / n)$ for the spectrum obtained by smashing $\mathcal{K}^{B M}(Y)$ with the mod- $n$ Moore spectrum, so that the $q$-th homotopy group of $\mathcal{K}^{B M}(Y ; \mathbb{Z} / n)$ is naturally isomorphic to $K_{q}^{\mathrm{BM}}(Y ; \mathbb{Z} / n)$.

Theorem 5.5. Let $W \subset Y$ be a closed embedding of Cohen-Macaulay, quasiprojective $k$-varieties and let $U=Y-Z$ denote the open complement.

(1) If $k$ is an algebraically closed field and $n \geq 1$ is an integer relatively prime to the exponential characteristic of $k$, then the natural maps of mod-n spectra

$$
\mathcal{K}^{B M}(W ; \mathbb{Z} / n) \rightarrow \mathcal{K}^{B M}(Y ; \mathbb{Z} / n) \rightarrow \mathcal{K}^{B M}(U ; \mathbb{Z} / n)
$$

form a homotopy fibration sequence of spectra.

(2) If $k$ is any field admitting resolutions of singularities, then the natural maps of spectra

$$
\mathcal{K}^{B M}(W) \rightarrow \mathcal{K}^{B M}(Y) \rightarrow \mathcal{K}^{B M}(U)
$$

form a homotopy fibration sequence of spectra.

Proof. Let $A$ denote $\mathbb{Z} / n$ or $\mathbb{Z}$. Embed $Y$ as an open subvariety of a projective $k$-variety $\bar{Y}$. By Corollary [5.3, it suffices to show

$$
\mathcal{K}_{g q f}((W, \bar{Y}) / \operatorname{Spec} k ; A) \rightarrow \mathcal{K}_{g q f}((Y, \bar{Y}) / \operatorname{Spec} k ; A) \rightarrow \mathcal{K}_{g q f}((U, \bar{Y}) / \operatorname{Spec} k ; A)
$$

is a homotopy fibration sequence of spectra. Since a degree-wise fibration sequence of simplicial spectra induces a homotopy fibration sequence on geometric realizations (cf. [5]), it suffices to show that for any smooth, quasi-projective $k$-variety $X$, the natural maps

$$
\mathcal{K}^{q f}(X,(W, \bar{Y}) ; A) \rightarrow \mathcal{K}^{q f}(X,(Y, \bar{Y}) ; A) \rightarrow \mathcal{K}^{q f}(X,(U, \bar{Y}) ; A)
$$

form a homotopy fibration sequence. Using Proposition 5.4, it suffices to show

$$
\mathcal{G}^{q f}(X,(W, \bar{Y}) ; A) \rightarrow \mathcal{G}^{q f}(X,(Y, \bar{Y}) ; A) \rightarrow \mathcal{G}^{q f}(X,(U, \bar{Y}) ; A)
$$

is a homotopy fibration sequence. This follows from Lemma 5.1 .

The following result shows that $\mathcal{K}^{B M}$ really is the Borel-Moore theory associated to $\mathcal{K}^{\text {hom }}$ (at least on the category of Cohen-Macaulay varieties).

Corollary 5.6. Let $W \subset Y$ be a closed embedding of Cohen-Macaulay, projective $k$-varieties and set $U=Y-W$.

(1) If $k$ is an algebraically closed field and $n \geq 1$ is an integer relatively prime to the exponential characteristic of $k$, then the natural maps of mod-n spectra

$$
\mathcal{K}^{\text {hom }}(W ; \mathbb{Z} / n) \rightarrow \mathcal{K}^{\text {hom }}(Y ; \mathbb{Z} / n) \rightarrow \mathcal{K}^{B M}(U ; \mathbb{Z} / n)
$$

form a homotopy fibration sequence of spectra.

(2) If $k$ is any field admitting resolutions of singularities, then the natural maps of spectra

$$
\mathcal{K}^{\text {hom }}(W) \rightarrow \mathcal{K}^{\text {hom }}(Y) \rightarrow \mathcal{K}^{B M}(U)
$$

form a homotopy fibration sequence of spectra.

Proof. This is an immediate consequence of the theorem and Proposition 3.12 


\section{Gersten COnjecture}

In this section we establish the analogue of the so-called Gersten conjecture in $K$ theory for the collection of contravariant functors $K_{m}^{q f}(-,(U, Y)), m \geq 0$. The main result of this section will be used to understand the local behavior of the presheaf $K_{m}^{q f}(-,(U, Y))$ in the proof of Theorem 7.1 below. To state the result in its natural level of generality, we need the following new definition. Throughout this section, the reader might wish to consider initially the special case when $U=Y=$ Spec $k$, when all of the results, and indeed all of the proofs, reduce to those pertaining to the classical Gersten conjecture.

Definition 6.1. Let $Y$ be a projective $k$-variety, and let $U \subset Y$ be an open immersion. For any $k$-scheme $X$, let $\mathcal{M}^{q f, t}(X,(U, Y))$ denote the abelian category consisting of those coherent sheaves $M$ on $X \times U$ which are supported on closed subschemes $Z$ satisfying (1) the closure $\bar{Z}$ of $Z$ in $X \times Y$ is finite over $X$ and (2) the image of $\pi: \bar{Z} \rightarrow X$ has codimension at least $t$ in $X$ (equivalently, the closure of the image of $Z$ in $X$ has codimension at least $t)$. Let $\mathcal{G}^{q f, t}(X,(U, Y))$ denote the associated $K$-theory spectrum.

Let $M \in \mathcal{M}^{q f, t}(X,(U, Y))$ with $Y$ an equidimensional projective $k$-variety, say of dimension $d$. Then $M$ is supported on a closed subscheme $Z \subset X \times U$ such that the closure $\bar{Z}$ of $Z$ in $X \times Y$ is finite over $X$. As shown in the proof of Proposition 5.4, for any choice of a finite, dominant morphism $\pi: Y \rightarrow \mathbb{P}^{d}$, the image of $\bar{Z}$ in $X \times \mathbb{P}^{d}$ is contained in a larger closed subscheme $(1 \times \pi)(Z) \subset W \subset X \times \mathbb{P}^{d}$ such that $W \rightarrow X$ is finite and flat. Upon pulling back $W$ to $X \times Y$, we see that $Z$ is contained in a larger closed subscheme $Z \subset Z^{\prime} \subset X \times Y$ such that $Z^{\prime} \rightarrow X$ is finite and dominant. Thus, in this situation, the category $\mathcal{M}^{q f, t}(X,(U, Y))$ can be described as the direct limit

$$
\underset{Z}{\lim } \mathcal{M}^{t}(Z)
$$

where the indexing ranges over those closed subschemes $Z \subset X \times U$ whose closures in $X \times Y$ are finite and dominant over $X$. Here, $\mathcal{M}^{t}(Z)$ denotes the abelian category of coherent sheaves on $Z$ whose supports have codimension at least $t$. This observation will be used in the proof of the following theorem.

Theorem 6.2. Let $X$ be a smooth, quasi-projective $k$-variety, $x=\left\{x_{1}, \ldots, x_{\nu}\right\} \subset$ $X$ a finite set of closed points, $Y$ an equidimensional projective $k$-variety, and $U \subset Y$ an open subscheme. Write $X_{x}$ for $\operatorname{Spec} \mathcal{O}_{X, x}$, where $\mathcal{O}_{X, x}$ is the semilocalization of $X$ at the finite set $x$. Then the map of $K$-theory spectra associated to the inclusion of abelian categories

$$
\mathcal{M}^{q f, t+1}\left(X_{x},(U, Y)\right) \longmapsto \mathcal{M}^{q f, t}\left(X_{x},(U, Y)\right)
$$

is weakly null-homotopic.

In particular, if $U$ is Cohen-Macaulay and $A$ is any abelian group, then for all $m \geq 0$, the natural map

$$
K_{m}^{q f}\left(X_{x},(U, Y) ; A\right) \longmapsto \bigoplus_{z \in X_{x} \times U} K_{m}(k(z) ; A)
$$

is an injection, where the direct sum is indexed by all points of $X_{x} \times U$ whose closures in $X_{x} \times Y$ are finite and dominant over $X_{x}$. If additionally $m=0$, this map is an isomorphism. 
Proof. The proof is essentially a souped-up version of Quillen's original proof of the Gersten conjecture (cf. [20, 5.11]). We follow the modification of Quillen's proof presented in [12]. In particular, we first assume $k$ is infinite.

As explained above, for any $d$ we have

$$
\mathcal{M}^{q f, d}\left(X_{x},(U, Y)\right)=\underset{Z}{\lim _{Z}} \mathcal{M}^{d}(Z)
$$

where the direct limit ranges over all those closed subschemes of $X_{x} \times U$ whose closures in $X_{x} \times Y$ are finite and dominant over $X_{x}$. To prove the theorem, it therefore suffices to fix such a subscheme $Z$ and prove the natural exact functor

$$
\mathcal{M}^{t+1}(Z) \rightarrow \mathcal{M}^{t}\left(X_{x},(U, Y)\right)
$$

induces a weak null-homotopic map on $K$-theory spectra. In fact, since $\mathcal{M}^{t+1}(Z)=$ $\lim _{Z^{\prime}} \mathcal{M}^{t}\left(Z^{\prime}\right)$ where the limit ranges over all closed subvarieties $Z^{\prime}$ of $Z$ of codimension one, it suffices to fix such a subvariety $Z^{\prime} \subset Z$ and prove that the natural map $\mathcal{G}^{t}\left(Z^{\prime}\right) \rightarrow \mathcal{G}^{q f, t}\left(X_{x},(U, Y)\right)$ is null-homotopic. (Here, $\mathcal{G}^{t}\left(Z^{\prime}\right)$ denotes the $K$-theory spectrum associated to $\mathcal{M}^{t}\left(Z^{\prime}\right)$.)

Upon replacing $X$ with a sufficiently small open neighborhood of $x$ in $X$, we may assume that $Z \rightarrow X$ is quasi-finite and that its closure in $X \times Y$ is finite and dominant over $X$. Modifying our previous notation for simplicity by replacing $X$ with a suitably small open neighborhood of $x$, let us now write $Z \subset X \times U$ for a closed subscheme whose closure $\bar{Z}$ in $X \times Y$ is finite and dominant over $X$. Let $Z^{\prime} \subset Z$ be a codimension one subscheme and let $\bar{Z}^{\prime}$ denote its closure in $X \times Y$. Finally, we will write $Z_{x}, Z_{x}^{\prime}, \bar{Z}_{x}$, and $\bar{Z}_{x}^{\prime}$ for the pullbacks of the evident schemes along $X_{x} \rightarrow X$. To prove the theorem, it suffices to show the natural functor $j_{*}: \mathcal{M}^{t}\left(Z_{x}^{\prime}\right) \rightarrow \mathcal{M}^{q f, t}\left(X_{x},(U, Y)\right)$, where $j: Z_{x}^{\prime} \longmapsto X_{x} \times U$ is the canonical closed immersion, induces a weak null-homotopy on associated $K$-theory spectra.

Observe that the image of $\bar{Z}^{\prime}$ in $X$ has codimension one. As shown in [12, 1.3], since $X$ is smooth (say of dimension $d$ ), there exists a map $\pi: X \rightarrow \mathbb{A}^{d-1}$ which is smooth near $x$ and such that the composition of $\bar{Z}^{\prime} \rightarrow X \rightarrow \mathbb{A}^{d-1}$ is a finite map. Let $\bar{W}^{\prime}=X \times_{\mathbb{A}^{d-1}} \bar{Z}^{\prime}$ and $W^{\prime}=X \times_{\mathbb{A}^{d-1}} Z^{\prime}$. Then there is a pullback square of the form

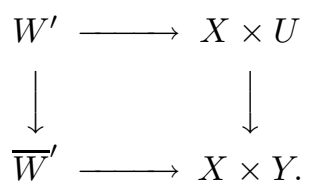

The bottom map in this diagram is finite since its composition with the projection $X \times Y \rightarrow X$ is finite. Thus the map $W^{\prime} \rightarrow X \times U$ is also finite. By construction, there is a natural map $q: W^{\prime} \rightarrow Z^{\prime}$ which is split by a closed immersion $i$ : $Z^{\prime} \rightarrow W^{\prime}$. Moreover, the map $q$ is smooth near each of the finitely many points $Q=\left\{w_{1}^{\prime}, \ldots, w_{m}^{\prime}\right\}$ comprising the fiber of $W^{\prime} \rightarrow X$ over $x$. It follows that the closed subscheme $j\left(Z^{\prime}\right) \subset W^{\prime}$ is a Cartier divisor near each point of $Q \cap j\left(Z^{\prime}\right)$, and is thus defined by a single non-zero divisor in some neighborhood of $Q$ in $W^{\prime}$. We thus have a short exact sequence of $\mathcal{O}_{W^{\prime}, Q^{-} \text {-modules }}$

$$
0 \rightarrow \mathcal{O}_{W^{\prime}, Q} \rightarrow \mathcal{O}_{W^{\prime}, Q} \rightarrow \mathcal{O}_{Z^{\prime}, Q \cap Z^{\prime}} \rightarrow 0
$$

The composition $Z^{\prime} \longmapsto W^{\prime} \rightarrow X \times U$ coincides with the original closed immersion $j: Z^{\prime} \longmapsto X \times U$, and thus $\mathcal{O}_{Z^{\prime}, Q \cap Z^{\prime}}=\mathcal{O}_{Z^{\prime}, x}$. Note that $\mathcal{O}_{W^{\prime}, Q}$ is $\mathcal{O}_{Z^{\prime}, x}$-flat and 
consequently we have a short exact sequence of exact functors from $\mathcal{M}^{t}\left(Z_{x}^{\prime}\right)$ to $\mathcal{M}^{t}\left(W_{Q}^{\prime}\right)$ given on an object $M \in \mathcal{M}^{t}\left(Z_{x}^{\prime}\right)$ by

$$
0 \rightarrow M \otimes_{\mathcal{O}_{Z^{\prime}, x}} \mathcal{O}_{W^{\prime}, Q} \rightarrow M \otimes_{\mathcal{O}_{Z^{\prime}, x}} \mathcal{O}_{W^{\prime}, Q} \rightarrow i_{*} M \rightarrow 0 .
$$

From above, the map $W^{\prime} \rightarrow X \times U$ is finite and thus so is the localization $g: W_{Q}^{\prime} \rightarrow$ $X_{x} \times U$. Taking pushforwards we have a short exact sequence of exact functors $\mathcal{M}^{t}\left(Z_{x}\right) \rightarrow \mathcal{M}^{q f, t}\left(X_{x},(U, Y)\right)$ given on an object $M$ by

$$
0 \rightarrow g_{*}\left(M \otimes_{\mathcal{O}_{Z^{\prime}}} \mathcal{O}_{W^{\prime}, Q}\right) \rightarrow g_{*}\left(M \otimes_{\mathcal{O}_{Z^{\prime}}} \mathcal{O}_{W^{\prime}, Q}\right) \rightarrow j_{*} M \rightarrow 0 .
$$

(Note that $g_{*}\left(M \otimes \mathcal{O}_{Z^{\prime}, x} \mathcal{O}_{W^{\prime}, Q}\right)$ is supported on the image of $\bar{W}^{\prime}$ and hence lies in $\mathcal{M}^{q f, t}\left(X_{x},(U, Y)\right)$.) Using Quillen's Additivity Theorem [20, Theorem 2], this proves the map $j_{*}: \mathcal{G}^{t}\left(Z_{x}^{\prime}\right) \rightarrow \mathcal{G}^{q f, t}\left(X_{x},(U, Y)\right)$ is null-homotopic.

The case of a finite field $k$ is deduced from the infinite case in precisely the same fashion as in [12.

To prove the final two assertions, define an exact functor

$$
F: \mathcal{M}^{q f}\left(X_{x},(U, Y)\right) \rightarrow \bigoplus_{z} \mathcal{M}^{\text {fin.len. }}\left(\mathcal{O}_{z}\right)
$$

by sending $M$ to $\left(M_{z}\right)_{z}$, where $z$ ranges over points of $X_{x} \times U$ as in the statement of the theorem and $\mathcal{M}^{\text {fin.len. }}$ denotes the abelian category of finitely generated modules of finite length. We claim that the functor (10) is "surjective up to isomorphism", in the sense that for every object $\left(M_{z}\right)$ of $\bigoplus \mathcal{M}^{\text {fin.len. }}\left(\mathcal{O}_{z}\right)$, there is an object $N$ of $\mathcal{M}^{q f}\left(X_{x},(U, Y)\right)$ and isomorphisms $M_{z} \cong N_{z}=N \otimes_{\mathcal{O}_{X_{x} \times U}} \mathcal{O}_{X_{x} \otimes U, z}$ and, furthermore, for every map $\left(M_{z}\right) \rightarrow\left(M_{z}^{\prime}\right)$ in $\bigoplus_{z} \mathcal{M}^{\text {fin.len. }}\left(\mathcal{O}_{z}\right)$ there is a map $N \rightarrow N^{\prime}$ in $\mathcal{M}^{q f}\left(X_{x},(U, Y)\right)$ such that $M_{z} \cong N_{z}, M_{z}^{\prime} \cong N_{z}^{\prime}$, and the evident triangles commute. To see this, note that if $M_{z}$ is in $\mathcal{M}^{\text {fin.len. }}\left(\mathcal{O}_{X_{x} \times U, z}\right)$, then $M_{z}$ is supported on $\mathcal{O}_{\tilde{Z}, z}$ where $\tilde{Z}$ is an infinitesimal thickening of $Z$ (i.e., if $\mathcal{I}$ is the sheaf of ideals definition $Z \subset X \times U$, then let $\tilde{Z}$ be defined by $\mathcal{I}^{n}$ for $n \gg 0$ ). Since the closure of $\tilde{Z}$ in $X \times Y$ is also finite over $X$, the desired surjectivity holds.

It follows that the functor $F$ identifies the abelian category $\bigoplus_{z} \mathcal{M}^{\text {fin.len. }}\left(\mathcal{O}_{z}\right)$ with the quotient abelian category of $\mathcal{M}^{q f}\left(X_{x},(U, Y)\right)$ by the Serre subcategory $\mathcal{M}^{q f, 1}\left(X_{x},(U, Y)\right)$. Thus by [20, Theorem 5] there is a homotopy fibration sequence of spectra

$$
\mathcal{G}^{q f, 1}\left(\left(U_{x}, Y_{x}\right) / X_{x}\right) \rightarrow \mathcal{G}^{q f}\left(\left(U_{x}, Y_{x}\right) / X_{x}\right) \rightarrow \bigoplus_{z} \mathcal{G}^{\text {fin.len. }}\left(\mathcal{O}_{z}\right) .
$$

Since the exact functor defined by pushforward

$$
\bigoplus_{z} \mathcal{M}(k(z)) \rightarrow \bigoplus_{z} \mathcal{M}^{\text {fin.len. }}\left(\mathcal{O}_{z}\right)
$$

induces a weak equivalence on $K$-theory spectra by [20, Theorem 4], the injectivity of

$$
K_{m}^{q f}\left(X_{x},(U, Y) ; A\right) \longmapsto \bigoplus_{z \in X_{x} \times U} K_{m}(k(z) ; A)
$$

follows from the first part of the theorem and Proposition 5.4. Finally, when $m=0$ and $A=\mathbb{Z}$, the target of (11) is isomorphic to the free abelian group on the set of closed, integral subschemes of $X_{x} \times U$ whose closures in $X_{x} \times Y$ are finite and dominant over $X_{x}$. Given such a subscheme $Z$, its structure sheaf $\mathcal{O}_{Z}$ determines a class in $K_{0}^{q f}\left(X_{x},(U, Y)\right)$ which clearly maps to the basis element [Z] 
of this free abelian group, and thus (11) is surjective in this case. For a general $A$ with $m=0$, observe that $K_{0}^{q f}\left(X_{x},(U, Y) ; A\right)=K_{0}^{q f}\left(X_{x},(U, Y)\right) \otimes A$ and that $\bigoplus_{z} K_{0}(k(z) ; A) \cong\left(\bigoplus_{z} K_{0}(k(z))\right) \otimes A$, since the underlying spectra are connective.

Remark 6.3. The spectra $\mathcal{G}^{q f, t}(X,(U, Y))$ form a decreasing tower starting with $\mathcal{G}^{q f}(X,(U, Y))$

$$
\mathcal{G}^{q f, d}(X,(U, Y)) \rightarrow \cdots \rightarrow \mathcal{G}^{q f, 1}(X,(U, Y)) \rightarrow \mathcal{G}^{q f, 0}(X,(U, Y))=\mathcal{G}^{q f}(X,(U, Y)),
$$

where $d=\operatorname{dim}(X)$. Letting $\mathcal{M}^{q f, t / t+1}(X,(U, Y))$ denote the quotient of the abelian category $\mathcal{M}^{q f, t}(X,(U, Y))$ by the Serre subcategory $\mathcal{M}^{q f, t+1}(X,(U, Y))$ and letting $\mathcal{G}^{q f, t / t+1}(X,(U, Y))$ denote the associated $K$-theory spectrum, we obtain the existence of a complex of abelian groups

$$
\begin{gathered}
0 \rightarrow G_{m}^{q f}(X,(U, Y)) \rightarrow G_{m}^{q f, 0 / 1}(X,(U, Y)) \rightarrow G_{m-1}^{q f, 1 / 2}(X,(U, Y)) \rightarrow \cdots \\
\rightarrow G_{0}^{q f, m / m+1}(X,(U, Y)) \rightarrow 0
\end{gathered}
$$

(where $G_{i}^{q f, t / t+1}(X,(U, Y))=0$ if $\left.t>\operatorname{dim}(X)\right)$. Moreover, this complex is natural for quasi-finite, flat maps and in particular may be regarded as a complex of presheaves for the small Zariski (or étale) site on a fixed $X$. For $X$ smooth, the theorem implies that this complex is exact locally in Zariski topology.

As was also proven in the theorem, when $U$ is Cohen-Macaulay, the presheaf $G_{m}^{q f, 0 / 1}(-,(U, Y))$ (defined on the small Zariski site on over a smooth, connected variety $X$ ) is locally isomorphic to $\bigoplus_{z \in X \times U} K_{m}(k(z))$, where $z$ ranges over points whose closures in $X \times Y$ are finite and dominant over $X$. More generally, write $P^{t}(X,(U, Y))$ for the collection of points $z \in X \times U$ such that the closure of $z$ in $X \times Y$ is finite over $X$ and the image of $z$ in $X$ has codimension $t$. Then it is not hard to prove $G_{m}^{q f, t / t+1}(-,(U, Y))$ is locally isomorphic to $\bigoplus_{z \in P^{t}(X,(U, Y))} K_{m}(k(z))$. The latter presheaf is a sheaf on the small Zariski site over $X$ for all $t$ and $m$, a fact which is seen to hold by observing that the covariant set-valued functor $(V \subset X) \mapsto$ $P^{t}(V,(U, Y))$ satisfies the dual of the sheaf axiom. Thus we obtain a resolution of the sheafification of $K_{m}^{q f}(-,(U, Y))$ of the form

$$
\begin{array}{r}
0 \rightarrow K_{m}^{q f}(-,(U, Y))_{\tilde{Z} a r} \rightarrow \bigoplus_{z \in P^{0}(X,(U, Y))} K_{m}(k(z)) \rightarrow \bigoplus_{z \in P^{1}(X,(U, Y))} K_{m-1}(k(z)) \\
\rightarrow \cdots \rightarrow \bigoplus_{z \in P^{m-1}(X,(U, Y))} K_{1}(k(z)) \rightarrow \bigoplus_{z \in P^{m}(X,(U, Y))} K_{0}(k(z)) \rightarrow 0 .
\end{array}
$$

Taking $U=Y=\operatorname{Spec} k$, this recovers Quillen's well-known resolution of $K_{m}(-) \tilde{Z}_{a r}$ (cf. [20, 5.9]), which leads to a proof of Bloch's formula:

$$
\mathrm{H}_{Z a r}^{m}\left(X, K_{m}(-) \tilde{Z}_{a r}\right) \cong C H^{m}(X) \text {. }
$$

Unfortunately, unlike in the special case $U=Y=\operatorname{Spec} k$, the sheaf

$$
X \mapsto \bigoplus_{z \in P^{t}(X,(U, Y))} K_{m}(k(z))
$$

is in general not flasque, since for a open subscheme $V \subset X$ (with $V \neq X$ ), the set $P^{t}(V,(U, Y))$ is strictly larger than $P^{t}(X,(U, Y))$. Thus one apparently cannot obtain, in general, a formula for the $m$-th Zariski cohomology group of $K_{m}^{q f}(-,(U, Y)) \tilde{Z}_{a r}$ analogous to Bloch's formula. 
As an application (unrelated to the remainder of this paper) of the machinery developed in this section and the previous ones, we provide the following two theorems. Together they relate the motivic complexes of Voevodsky with certain complexes defined by Grayson [14] involving $K_{0}$ groups of automorphisms (see Theorem 6.5). For any $k$-variety $Y$, define $c_{\text {equi }}^{0}(-, Y)$ to be the presheaf on $S c h / k$ whose value at $X$ is $c_{\text {equi }}^{0}(X, Y)=c_{\text {equi }}^{0}(X \times Y / X)$, the free abelian group on the collection of closed, integral subschemes of $X \times Y$ that are finite and dominant over some irreducible component of $X$.

Theorem 6.4. Let $P$ be a Cohen-Macaulay, projective $k$-variety, where $k$ is an arbitrary field. Let

$$
K_{0}^{f i n}(-, P) \rightarrow c_{\text {equi }}^{0}(-, P)
$$

be the map of presheaves on $S c h / k$ defined at a variety $X$ by sending the class of a module $M \in \mathcal{P}^{\text {fin }}(X, P)$ to the cycle $\sum_{z} \operatorname{length}_{\mathcal{O}_{X \times P, z}}\left(M_{z}\right)[\bar{z}]$, where the sum ranges over points $z$ of $X \times P$ whose closures are finite and dominant over an irreducible component of $X$. Then the induced map

$$
K_{0}^{f i n}\left(-\times \Delta^{\bullet}, P\right) \rightarrow c_{e q u i}^{0}\left(-\times \Delta^{\bullet}, P\right)
$$

is a homotopy equivalence locally in the Zariski topology on $\mathrm{Sm} / \mathrm{k}$.

Proof. By Theorems 4.24 and 4.22 it suffices to prove the natural map

$$
K_{0}^{f i n}(-, P) \rightarrow c_{e q u i}^{0}(-, P)
$$

is an isomorphism locally for the Nisnevich topology on $S m / k$. (One may readily verify that this morphism is a morphism of presheaves with $K_{0}$ transfers.) In fact, we show it is so for the Zariski topology. Let $X$ belong to $S m / k$, let $x$ be a point in $X$, and define $X_{x}=\operatorname{Spec} \mathcal{O}_{X, x}$, the spectrum of the local ring of $X$ at $x$. It suffices to prove $K_{0}^{f i n}\left(X_{x}, P\right) \rightarrow c_{\text {equi }}^{0}\left(X_{x}, P\right)$ is an isomorphism. This is precisely the final assertion of Theorem 6.2 (for $Y=U=P$ and $A=\mathbb{Z}$ ).

Define $K_{0}^{f i n}\left(-, \mathbb{G}_{m}^{\wedge t}\right)$ to be the the quotient presheaf of $K_{0}^{f i n}\left(-, \mathbb{G}_{m}^{t}\right)$ given by factoring out the images of

$$
K_{0}^{f i n}\left(-, \mathbb{G}_{m}^{i} \times\{1\} \times \mathbb{G}_{m}^{t-i-1}\right) \rightarrow K_{0}^{f i n}\left(-, \mathbb{G}_{m}^{t}\right),
$$

for $i=0, \ldots, t-1$, induced by the closed immersions $\mathbb{G}_{m}^{i} \times\{1\} \times \mathbb{G}_{m}^{t-i-1} \mapsto \mathbb{G}_{m}^{t}$. One may readily verify that the $t$ projection maps of the form $\mathbb{G}_{m}^{t} \rightarrow \mathbb{G}_{m}^{t-1}$ induce a family of compatible splitting so that $K_{0}^{f i n}\left(-, \mathbb{G}_{m}^{\wedge t}\right)$ is a summand of $K_{0}^{f i n}\left(-, \mathbb{G}_{m}^{t}\right)$. Define $c_{\text {equi }}^{0}\left(-, \mathbb{G}_{m}^{\wedge t}\right)$ similarly, and observe that the same splittings show it is a summand of $c_{\text {equi }}^{0}\left(-, \mathbb{G}_{m}^{t}\right)$.

Theorem 6.5. The natural map

$$
K_{0}^{f i n}\left(-\times \Delta^{\bullet}, \mathbb{G}_{m}^{\wedge t}\right) \rightarrow c_{\text {equi }}^{0}\left(-\times \Delta^{\bullet}, \mathbb{G}_{m}^{\wedge t}\right)
$$

is a homotopy equivalence locally in the Zariski topology on $\mathrm{Sm} / \mathrm{k}$.

Proof. Since the given map is a summand of the map

$$
K_{0}^{f i n}\left(-\times \Delta^{\bullet}, Y_{1} \times \cdots \times Y_{t}\right) \rightarrow c_{\text {equi }}^{0}\left(-\times \Delta^{\bullet}, Y_{1} \times \cdots \times Y_{t}\right),
$$

where $Y_{i}=\mathbb{G}_{m}^{1}$ for all $i$, it suffices to prove (12) is a homotopy equivalence locally for the Zariski topology on $S m / k$, where each $Y_{i}$ is $\mathbb{A}^{1}, \mathbb{G}_{m}^{1}$ or $\mathbb{P}^{1}$. 
As a preliminary step, we contend that if $V=U_{0} \cup U_{1}$ is an open covering of a quasi-projective variety $V$, then each of the squares of chain complexes of sheaves

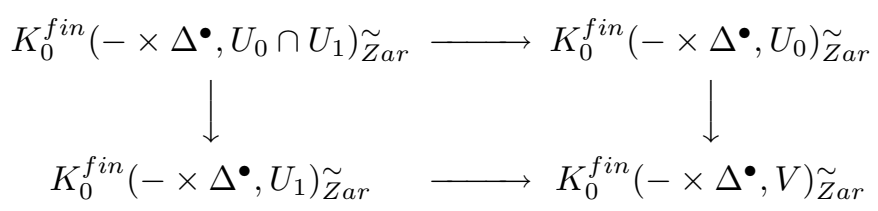

and

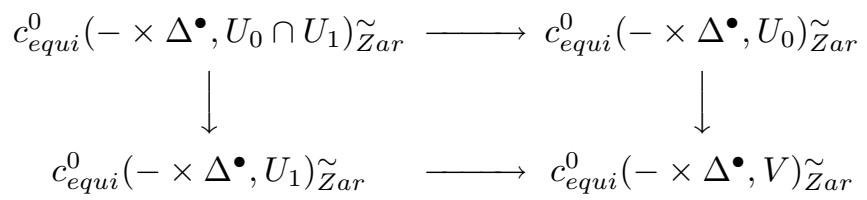

has the property that the induced map on the cones of the columns is a homotopy equivalence. By Theorems 4.24 and 4.22 it suffices to prove the induced map on the cones of the columns of

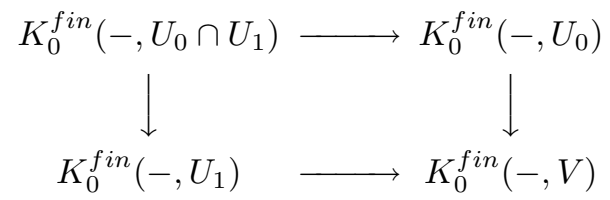

is locally an isomorphism for the Nisnevich topology, and similarly for the diagram obtained by replacing $K_{0}^{f i n}$ by $c_{\text {equi }}^{0}$. Let $X$ be a smooth variety, let $x$ be a point of $X$, and let $X_{x}^{\text {hen }}$ denote the spectrum of the henselization of the local ring of $X$ at $x$. Observe that, for any open subscheme $W$ of $V, K_{0}^{f i n}\left(X_{x}^{h e n}, W\right)=$ $\lim _{Z} K_{0}^{f i n}\left(Z / X_{x}^{\text {hen }}\right)$, where $Z$ ranges over closed subschemes of $X_{x}^{\text {hen }} \times W$ that are finite and dominant over $X_{x}^{\text {hen }}$. Since any such $Z$ decomposes as a disjoint union of spectra of local rings, we have

$$
K_{0}^{f i n}\left(X_{x}^{h e n}, W\right) \cong \lim _{Z} K_{0}^{f i n}\left(Z \cap W / X_{x}^{h e n}\right)
$$

where the limit ranges over closed subschemes $Z$ of $X_{x}^{\text {hen }} \times V$ that are finite and dominant over $X_{x}^{\text {hen }}$. The desired result follows. A similar (in fact, simpler) argument applies for $c_{\text {equi }}^{0}$.

Returning our attention to the map (12), note that it is locally a homotopy equivalence if each $Y_{i}$ is $\mathbb{P}^{1}$ by Theorem 6.4 Moreover, for any $W$, a standard argument shows that the maps

$$
K_{0}^{f i n}\left(-\times \Delta^{\bullet}, W \times \mathbb{A}^{1}\right) \rightarrow K_{0}^{f i n}\left(-\times \Delta^{\bullet}, W\right)
$$

and

$$
c_{\text {equi }}^{0}\left(-\times \Delta^{\bullet}, W \times \mathbb{A}^{1}\right) \rightarrow c_{\text {equi }}^{0}\left(-\times \Delta^{\bullet}, W\right)
$$

defined by pushforward along $W \times \mathbb{A}^{1} \rightarrow W$ are homotopy equivalences. Thus (12) is a homotopy equivalence if each $Y_{i}$ is either $\mathbb{P}^{1}$ or $\mathbb{A}^{1}$. We proceed by induction on the number $N$ of copies of $\mathbb{G}_{m}^{1}$ appearing in the list $Y_{1}, \ldots, Y_{t}$. For $N>0$, after re-indexing, we may assume that $Y_{1}=\mathbb{G}_{m}^{1}$. Let $Y=Y_{2} \times \cdots \times Y_{t}$ and let $\mathbb{P}^{1}=\mathbb{A}^{1} \cup \mathbb{A}^{1}$ denote the standard open cover. Then the general result proven above 
shows that the induced map on the cones of the columns of

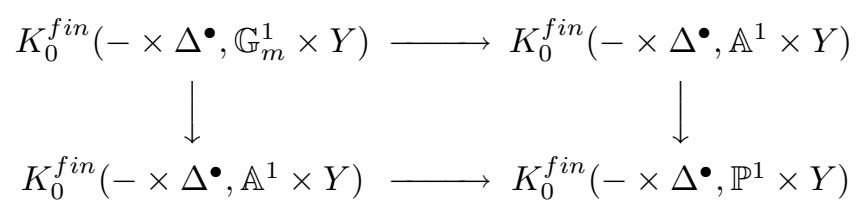

is locally a homotopy equivalence for the Zariski topology, and similarly for $c_{\text {equi }}^{0}$ replacing $K_{0}^{f i n}$. Using the naturality of $K_{0}^{f i n}(-, W) \rightarrow c_{\text {equi }}^{0}(-, W)$ for open immersions $W \longmapsto W^{\prime}$, the proof is complete.

\section{7. ÉtALE DESCENT FOR $K$-HOMOLOGY}

In this section we establish the existence of a natural convergent spectral sequence abutting to $K_{*}^{\mathrm{BM}}(U ; \mathbb{Z} / n)$ whose $E_{2}$ terms are given by étale cohomology groups (see Theorem [7.6). Here, $U$ is assumed to be a Cohen-Macaulay variety defined over an algebraically closed field $k$ and $n \geq 1$ is an integer relatively prime to the exponential characteristic of $k$. This spectral sequence should be thought of as the analogue for $\mathcal{K}^{B M}$ with finite coefficients of the motivic Atiyah-Hirzebruch spectral sequence [3, 8, 17]. We also show $\mathcal{K}^{B M}(-; \mathbb{Z} / n)$ satisfies étale descent in the sense described in Theorem7.8 below.

A key ingredient in both of these theorems is Suslin's theorem relating motivic cohomology of weight at least $\operatorname{dim}(U)$ with étale cohomology. In fact, we need a slight extension of Suslin's result involving a naturality assertion not present in his original formulation. This is given in Theorem 7.3. Another key ingredient is given by the following result.

Theorem 7.1. Let $U$ be a Cohen-Macaulay, quasi-projective variety over an algebraically closed field $k$ and assume $n \geq 1$ is an integer relatively prime to the exponential characteristic of $k$. Then for all $m \geq 0$, the map of presheaves

$$
K_{0}^{q f}(-, U) \otimes K_{m}(k ; \mathbb{Z} / n) \rightarrow K_{m}^{q f}(-, U ; \mathbb{Z} / n)
$$

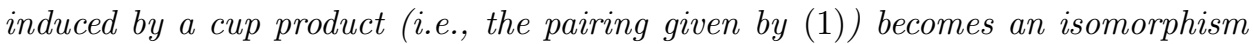
upon sheafification in the h-topology. Moreover, this isomorphism is contravariantly natural for quasi-finite, flat morphisms of Cohen-Macaulay varieties.

Proof. Let $U \subset Y$ be an open dense embedding with $Y$ projective. Using Proposition 5.2 it suffices to show the map defined by cup product

$$
\theta: K_{0}^{q f}(-,(U, Y)) \otimes K_{m}(k ; \mathbb{Z} / n) \rightarrow K_{m}^{q f}(-,(U, Y) ; \mathbb{Z} / n)
$$

becomes an isomorphism upon sheafification in the $h$-topology. For any integral $k$-scheme $X$, we have a commutative diagram of the form

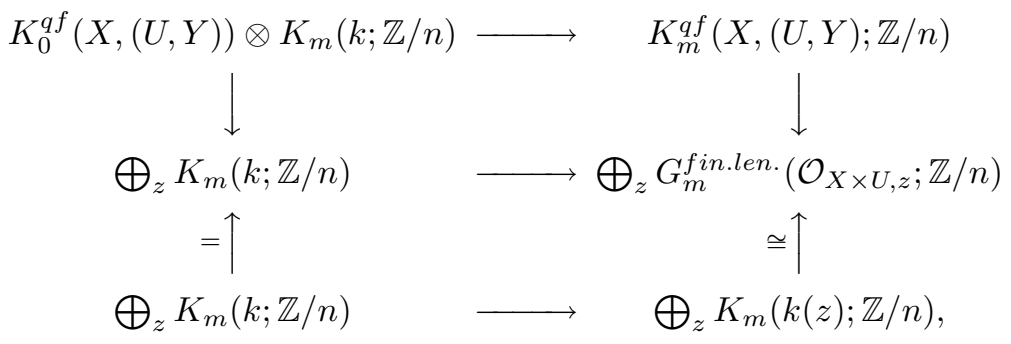


where $z$ ranges over all points of $X \times U$ whose closures in $X \times Y$ are finite and dominant over $X$. The upper-left vertical map in this diagram is defined by tensoring $K_{m}(k ; \mathbb{Z} / n)$ with the cycle class map $K_{0}^{q f}(X,(U, Y)) \rightarrow \bigoplus_{z} \mathbb{Z}$ taking $M$ to $\sum_{z}$ length $_{z}\left(M_{z}\right)$. The upper-right vertical map is given by the evident localization functor, and the bottom horizontal map is induced by the field extensions $k \longmapsto k(z)$. If $X$ is the localization of a smooth variety at a closed point, then by Theorem 6.2 the upper-right vertical map of (14) is an injection and the upper-left vertical map is an isomorphism.

Let $f: X^{\prime} \rightarrow X$ be a dominant map of smooth, connected varieties of the same dimension and let $x$ and $x^{\prime}$ denote the generic points of $X$ and $X^{\prime}$. In this situation, we define two pullback maps

$$
f^{*}: \bigoplus_{z} K_{m}(k ; \mathbb{Z} / n) \rightarrow \bigoplus_{z^{\prime}} K_{m}(k ; \mathbb{Z} / n)
$$

and

$$
f^{*}: \bigoplus_{z} K_{m}(k(z) ; \mathbb{Z} / n) \rightarrow \bigoplus_{z^{\prime}} K_{m}\left(k\left(z^{\prime}\right) ; \mathbb{Z} / n\right)
$$

with $z$ (resp., $z^{\prime}$ ) ranging over points of $X \times Y$ (resp., $X^{\prime} \times Y$ ) whose closures in $X \times Y$ (resp., $\left.X^{\prime} \times Y\right)$ are finite and dominant over $X$ (resp., $X^{\prime}$ ). To define the latter map $f^{*}$, given such a $z$, let $z_{1}^{\prime}, \ldots, z_{l}^{\prime}$ be the finitely many points which belong to the fiber of $X^{\prime} \times U \rightarrow X \times U$ over $z$ and whose closures in $X^{\prime} \times Y$ are finite and dominant over $X^{\prime}$. (That there are only finitely many such points follows from the fact that $k(x) \longmapsto k(z)$ and $k(x) \longmapsto k\left(x^{\prime}\right)$ are finite field extensions.) Then the map $f^{*}: K_{m}(k(z) ; \mathbb{Z} / n) \rightarrow \bigoplus_{z^{\prime}} K_{m}\left(k\left(z^{\prime}\right) ; \mathbb{Z} / n\right)$ is defined by the diagram

$$
K_{m}(k(z) ; \mathbb{Z} / n) \longrightarrow G_{m}^{\text {fin.len. }}\left(\mathcal{O}_{\operatorname{Spec} k(z) \times_{X} X^{\prime}} ; \mathbb{Z} / n\right) \stackrel{\cong}{\bigoplus_{i}} K_{m}\left(k\left(z_{i}^{\prime}\right) ; \mathbb{Z} / n\right) .
$$

The map $f^{*}: \bigoplus_{z} K_{m}(k ; \mathbb{Z} / n) \rightarrow \bigoplus_{z^{\prime}} K_{m}(k ; \mathbb{Z} / n)$ is defined by taking $m=0$ in the above situation and tensoring over $\mathbb{Z} / n$ by $K_{m}(k ; \mathbb{Z} / n)$.

We claim that the diagram (14) is functorial with respect to dominant morphisms of smooth, connected varieties of the same dimension. That is, given such a morphism $f: X^{\prime} \rightarrow X$, we claim that the cube

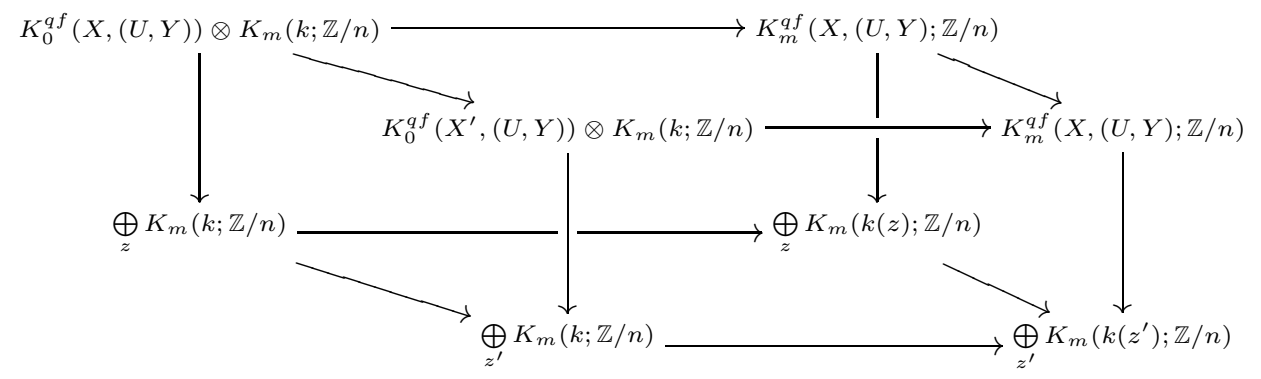

commutes. The front (resp., back) square of this cube are given by the outer square of (14) for the variety $X$ (resp., $X^{\prime}$ ), and the diagonal arrows are given by functoriality. The front, back, top, and bottom squares of (15) are readily seen to commute. The left-hand square commutes by the functoriality of the cycle class map. To establish the commutativity of the right-hand square, let $z \in X \times U$ and 
$z_{1}^{\prime}, \ldots, z_{l}^{\prime} \in X \times U$ be given as above. We claim that the diagram of spectra

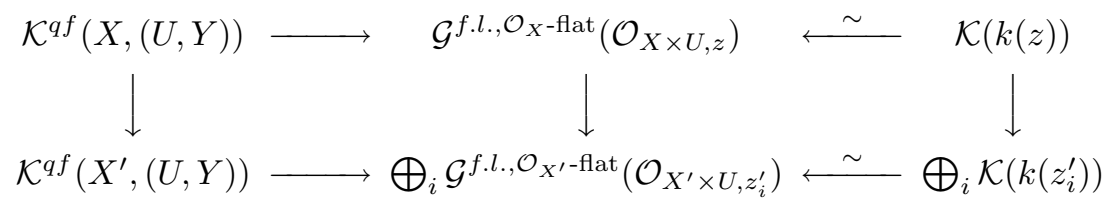

commutes. Here, $\mathcal{G}^{f . l ., \mathcal{O}_{X} \text {-flat }}\left(\mathcal{O}_{X \times U, z}\right)$ is the $K$-theory spectrum associated to the exact subcategory $\mathcal{M}^{\text {f.l., } \mathcal{O}_{X} \text {-flat }}\left(\mathcal{O}_{X \times U, z}\right)$ of $\mathcal{M}^{\text {fin.len. }}\left(\mathcal{O}_{X \times U, z}\right)$ consisting of coherent sheaves which are $\mathcal{O}_{X}$-flat. The spectrum $\mathcal{G}^{f . l ., \mathcal{O}_{X} \text {-flat }}\left(\mathcal{O}_{X^{\prime} \times U, z^{\prime}}\right)$ is similarly defined. (Note, in particular, that the middle vertical arrow of (16) is well-defined, since the underlying functor $-\otimes_{\mathcal{O}_{X}} \mathcal{O}_{X^{\prime}}$ is exact on $\mathcal{M}^{f . l ., \mathcal{O}_{X} \text {-flat }}\left(\mathcal{O}_{X \times U, z}\right)$.) Indeed, the commutativity of (16) is an immediate consequence of the commutativity of the diagram of schemes

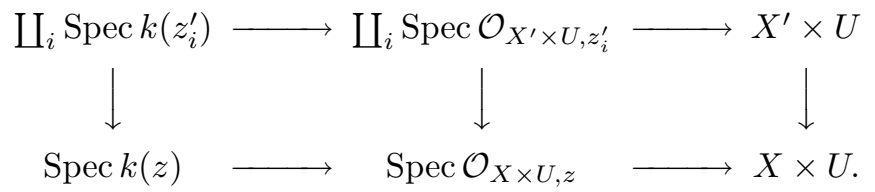

The fact that horizontal arrows on the right in (16) are weak equivalences as indicated follows from the fact that the natural map

$$
G^{f . l ., \mathcal{O}_{X} \text {-flat }}\left(\mathcal{O}_{X \times U, z}\right) \rightarrow G^{\text {fin.len. }}\left(\mathcal{O}_{X \times U, z}\right)
$$

is a weak equivalence by Quillen's Resolution Theorem [20, Theorem 3], and similarly for $X^{\prime}$ and $z^{\prime}$ in place of $X$ and $z$. Indeed, given a coherent sheaf $M$ in $\mathcal{M}^{\text {fin.len. }}\left(\mathcal{O}_{X \times Y, z}\right)$, as in the proof of Theorem 6.2, we have that $M$ is the localization sheaf $\tilde{M}$ in $\mathcal{M}^{q f}(X,(U, Y))$. As shown in the proof of Proposition 5.4 there exists a sheaf $E \in \tilde{\mathcal{P}^{q} f}(X,(U, Y))$ and a surjection $E \rightarrow \tilde{M}$. Localizing at $z$, we have a surjection $E_{z} \rightarrow M$ with $E_{z} \in \mathcal{M}^{f . l ., \mathcal{O}_{X} \text {-flat }}\left(\mathcal{O}_{X \times U, z}\right)$. Since $X$ is smooth, every object of $\mathcal{M}^{\text {fin.len. }}\left(\mathcal{O}_{X \times U, z}\right)$ thus admits a finite resolution by objects of $\mathcal{M}^{f . l ., \mathcal{O}_{X} \text {-flat }}\left(\mathcal{O}_{X \times U, z}\right)$. The commutativity of the right-hand square of (15) follows from (16).

We now show the map (13) becomes an injection upon $h$ sheafification. In fact, it is injective locally in the Zariski topology on the category of smooth varieties. Let $X$ be the localization of a smooth variety at a closed point. Then the upper vertical arrows of (14) are injections. The bottom arrow of (14) is injective since $k \longmapsto k(z)$ has finite transcendence degree and $k$ is algebraically closed. By de Jong's Theorem on alterations [7, every $k$-variety admits an $h$ covering by smooth varieties, and hence (13) is injective locally in the $h$-topology.

To prove the map (13) becomes a surjection upon $h$ sheafification, it suffices to pick a smooth, connected $k$-variety $X$ and an element $\alpha \in K_{m}^{q f}(X,(U, Y) ; \mathbb{Z} / n)$ and show that the pullback of $\alpha$ along some $h$ covering $\tilde{X} \rightarrow X$ lies in the image of $\theta_{\tilde{X}}$.

Referring to diagram (14), the image of $\alpha$ in $\bigoplus_{z} K_{m}(k(z) ; \mathbb{Z} / n)$ involves only finitely many $z$ 's - say $z_{1}, \ldots, z_{k}$ - and let this image be $\sum_{i} \alpha_{i}$, where $\alpha_{i} \in$ $K_{m}\left(k\left(z_{i}\right) ; \mathbb{Z} / n\right)$. By Suslin's theorem on the $K$-theory of algebraically closed fields 23], the map $K_{m}(k ; \mathbb{Z} / n) \rightarrow K_{m}(L ; \mathbb{Z} / n)$ is an isomorphism for any field extension $k \subset L$ with $L$ algebraically closed. Let $x$ denote the generic point of $X$ and choose an algebraic closure $\overline{k(x)}$ of $k(x)$ with $k\left(z_{i}\right) \subset \overline{k(x)}$ for all $i$. Then for each $\alpha_{i} \in K_{m}\left(k\left(z_{i}\right) ; \mathbb{Z} / n\right)$, there is a $\delta_{i} \in K_{m}(k ; \mathbb{Z} / n)$ such that the images of $\alpha_{i}$ and $\delta_{i}$ 
in $K_{m}(\overline{k(x)} ; \mathbb{Z} / n)$ are equal. There is thus a finite field extension $k(x) \subset F$ such that the images of $\alpha_{i}$ and $\delta_{i}$ in $K_{m}(F ; \mathbb{Z} / n)$ coincide, for all $i$.

We claim there is a dominant map $f: X^{\prime} \rightarrow X$ of smooth, connected $k$-varieties of the same dimension such that $F \subset k\left(x^{\prime}\right)$, where $x^{\prime}$ is the generic point of $X^{\prime}$. To see this, let $X^{\prime \prime}$ denote the integral closure of $X$ in $F$. By de Jong's Theorem [7], there is a dominant map $X^{\prime} \rightarrow X^{\prime \prime}$ with $X^{\prime}$ smooth and such that $\operatorname{dim}\left(X^{\prime \prime}\right)=$ $\operatorname{dim}\left(X^{\prime}\right)$, and we take $f$ to be the evident composition. Using the commutativity of $(15)$, we see that the pullback $f^{*}(\alpha)$ lies in the image of

$$
\bigoplus_{z^{\prime} \in X^{\prime} \times U} K_{m}(k ; \mathbb{Z} / n) \rightarrow \bigoplus_{z^{\prime} \in X^{\prime} \times U} K_{m}\left(k\left(z^{\prime}\right) ; \mathbb{Z} / n\right) .
$$

Using the facts that the upper-left (resp., upper-right) arrow of (14) is an isomorphism (resp., injective) locally in the Zariski topology on the smooth variety $X^{\prime}$, we may take $\tilde{X}=\coprod_{i} V_{i}$ for some finite Zariski open covering $X^{\prime}=\bigcup_{i} V_{i}$ of $X^{\prime}$.

The naturality assertion is evident since cupping with elements of $K_{m}(k ; \mathbb{Z} / n)$ commutes with flat pullback.

Theorem 7.1 together with Corollary 4.23 and Suslin's theorem identifying $K_{q}(k ; \mathbb{Z} / n)$ with $\mu_{n}^{\otimes q / 2}$ implies the existence of a spectral sequence abutting to $K_{*}^{\mathrm{BM}}(U ; \mathbb{Z} / n)$ whose $E_{2}$ terms are given by the homotopy groups of the family of simplicial abelian groups $d \mapsto K_{0}^{q f}\left(\Delta^{d}, U\right) \otimes \mu_{n}^{\otimes q / 2}, q \geq 0$. To relate these $E_{2}$ terms with étale cohomology, we use another theorem of Suslin, involving the presheaf $z_{\text {equi }}^{0}(-, Y)$ defined as follows.

Definition 7.2. For a quasi-projective $k$-variety $Y$, let $z_{\text {equi }}^{0}(-, Y)$ denote the presheaf on $S c h / X$ defined in [25, §3] whose value at a smooth variety $X$, $z_{\text {equi }}^{0}(X, Y)$, is the free abelian group of closed integral subschemes $Z$ of $X \times Y$ which are equi-dimensional of relative dimension 0 (i.e., quasi-finite and dominant) over a connected component of $X$ (cf. 25. 3.3.15]).

We write $c_{\text {equi }}^{0}(-, Y)$ for the sub-presheaf of $z_{\text {equi }}^{0}(-, Y)$ also defined in [25, §3] whose value at a smooth variety $X$ is a free abelian group of closed integral subschemes $Z$ of $X \times Y$ which are finite and dominant over a connected component of $X$.

For a morphism $f: X^{\prime} \rightarrow X$ of smooth varieties, the maps $f^{*}: z_{\text {equi }}^{0}(X, Y) \rightarrow$ $z_{\text {equi }}^{0}\left(X^{\prime}, Y\right)$ and $f^{*}: c_{\text {equi }}^{0}(X, Y) \rightarrow c_{\text {equi }}^{0}\left(X^{\prime}, Y\right)$ and are given by a pullback of cycles in the expected manner. Moreover, the collection of pairings

$$
c_{\text {equi }}^{0}(X / S) \otimes z_{\text {equi }}^{0}(X, Y) \rightarrow z_{\text {equi }}^{0}(S, Y)
$$

for $\pi: X \rightarrow S$ a smooth curve, defined by $z \otimes w \mapsto\left(\pi \times i d_{Y}\right)_{*}\left(p^{*}(z) \cap w\right)$ (where $p: X \times Y \rightarrow X$ is the canonical projection) endow $z_{\text {equi }}^{0}(-, Y)$ with the structure of a pretheory (see $[25,3.7 .5]$ ) and hence with the structure of a presheaf with $K_{0}$ transfers by Corollary 4.6 Moreover, these pairings restrict to pairings involving $c_{\text {equi }}^{0}(-, Y)$, so that $c_{\text {equi }}^{0}(-, Y)$ is a sub-pretheory of $z_{\text {equi }}^{0}(-, Y)$. Finally, using [25] 3.6.4], if $g: Y^{\prime} \rightarrow Y$ is a quasi-finite, flat morphism of varieties, there is a morphism of presheaves

$$
g^{*}: z_{\text {equi }}^{0}(-, Y) \rightarrow z_{\text {equi }}^{0}\left(-, Y^{\prime}\right),
$$

which upon restriction to a map of presheaves on $S m / k$ is given by a pullback of cycles. 
The presheaf $z_{\text {equi }}^{0}(-, U)$ is related to the étale cohomology of $U$ as shown in the following result which is due to Suslin. For an abelian group $A$, we write $A^{\#}$ for $\operatorname{Hom}_{\mathbb{Z}}(A, \mathbb{Q} / \mathbb{Z})$. Of course, if $A$ is an $\mathbb{Z} / n$-module for some $n \geq 1$, the group $A^{\#}$ coincides with $\operatorname{Hom}_{\mathbb{Z} / n}(A, \mathbb{Z} / n)$.

Theorem 7.3 (Suslin). Let $U$ be a quasi-projective variety defined over an algebraically closed field $k$ and assume $n \geq 1$ is an integer relatively prime to the exponential characteristic of $k$. Let $F$ be a finite $\mathbb{Z} / n$-module. Then there is an isomorphism

$$
\pi_{n}\left(z_{\text {equi }}^{0}\left(\Delta^{\bullet}, U\right) \otimes F\right) \cong \mathrm{H}_{c}^{n}\left(U, F^{\#}\right)^{\#} .
$$

This isomorphism is natural for quasi-finite, flat morphisms of normal varieties, where the functoriality of $\mathrm{H}_{c}^{n}\left(U, F^{\#}\right)^{\#}$ for such morphisms is given by applying $(-)^{\#}$ to the "trace" map in étale cohomology with compact supports (cf. [1, XVII. 6.2.7.5]).

Moreover, if $U$ is smooth of pure dimension $d$, then there is an isomorphism

$$
\pi_{n}\left(z_{\text {equi }}^{0}\left(\Delta^{\bullet}, U\right) \otimes F\right) \cong \mathrm{H}_{e ́ t}^{2 d-n}\left(U, F \otimes_{\mathbb{Z} / n} \mu_{n}^{\otimes d}\right)
$$

which is natural for quasi-finite, flat morphisms of smooth varieties.

Proof. Suslin's proof of [26, 4.1] applies directly to establish the existence of the asserted isomorphism if we use $F^{\#}$ coefficients in lieu of $\mathbb{Z} / n$ coefficients. Since we need the details of this proof in order to establish the asserted naturality, we recall Suslin's proof here. Begin by choosing an open, dense embedding $j: U \longmapsto \bar{U}$ with $\bar{U}$ projective and let $i: U_{\infty} \longmapsto \bar{U}$ be the (reduced) closed complement of $U$ in $\bar{U}$. Recall that $\mathrm{H}_{c}^{p}\left(U, F^{\#}\right)$ is by definition $\mathrm{H}_{\text {ét }}^{p}\left(U, j ! F^{\#}\right)$, where $j$ ! denotes extension by zero. By [25, 4.2.9 and 4.3.1], we have that

$$
0 \rightarrow z_{\text {equi }}^{0}\left(-, U_{\infty}\right)[1 / p]_{h}^{\sim} \stackrel{i_{*}}{\longrightarrow} z_{\text {equi }}^{0}(-, \bar{U})[1 / p]_{h}^{\sim} \stackrel{j^{*}}{\longrightarrow} z_{\text {equi }}^{0}(-, U)[1 / p]_{h}^{\sim} \rightarrow 0
$$

is an exact sequence of $h$ sheaves, where $p$ is the exponential characteristic of the ground field $k$. Moreover, $z_{\text {equi }}^{0}(-, Y)_{h}^{\sim}[1 / p] \cong \mathbb{Z}[1 / p]_{h}(Y)$ for any projective variety $Y$, where $\mathbb{Z}[1 / p]_{h}(Y)$ denotes the $h$ sheafification of $\mathbb{Z}[1 / p] \operatorname{Hom}(-, Y)$. An $h$ sheaf $\mathcal{G}$ on $S c h / k$ determines a collection of étale sheaves $\mathcal{G}_{X}$ on the small étale site $X_{\text {ét }}$, for all $X$ in $S c h / k$, together with canonical maps $\mathcal{F}_{X} \rightarrow f_{*} \mathcal{F}_{Y}$ and $f^{*} \mathcal{F}_{X} \rightarrow \mathcal{F}_{Y}$ for each morphism $f: Y \rightarrow X$. (The étale sheaf $\mathcal{G}_{X}$ is by definition the sheaf sending an étale map $W \rightarrow X$ to $\Gamma(W, \mathcal{G})$.) If $\mathcal{G}$ is any $h$ sheaf of $\mathbb{Z}[1 / p]$-modules, we therefore have the isomorphisms

$$
\begin{aligned}
\operatorname{Hom}_{h}\left(z_{\text {equi }}^{0}(-, U)[1 / p]_{h}^{\sim}, \mathcal{G}\right) & \cong \operatorname{ker}\left(\Gamma(\bar{U}, \mathcal{G}) \rightarrow \Gamma\left(U_{\infty}, \mathcal{G}\right)\right) \\
& \cong \operatorname{ker}\left(\Gamma\left(\bar{U}, \mathcal{G}_{\bar{U}}\right) \rightarrow \Gamma\left(\bar{U}, i_{*}\left(\mathcal{G}_{U_{\infty}}\right)\right)\right) .
\end{aligned}
$$

If $\mathcal{G}$ is constant, then we have in addition the isomorphism

$$
\operatorname{Hom}_{h}\left(z_{\text {equi }}^{0}(-, U)_{h}^{\sim}, \mathcal{G}\right) \cong \operatorname{ker}\left(\Gamma(\bar{U}, \mathcal{G}) \rightarrow \Gamma\left(\bar{U}, i_{*} \mathcal{G}\right)\right) \cong \Gamma\left(\bar{U}, j_{!} \mathcal{G}\right)
$$

Now choose an injective resolution $F^{\#} \rightarrow I^{\bullet}$ of the $h$ sheaf $F^{\#}$ consisting of $h$ sheaves of $\mathbb{Z}[1 / p]$-modules. Since, for all $Y$, the map $F^{\#} \rightarrow I_{Y}^{\bullet}$ defines an injective resolution of the constant sheaf $F^{\#}$ for the small étale site $Y_{\text {ét }}$, by using (19) we obtain that the canonical map from $j_{!} F^{\#}$ to the total complex associated to the surjection of chain complexes $I_{\bar{U}}^{\bullet} \rightarrow i_{*} I_{U_{\infty}}^{\bullet}$ is a quasi-isomorphism and hence determines an injective resolution of $j_{!} F^{\#}$ :

$$
j_{!} F^{\#} \stackrel{\sim}{\longrightarrow} \operatorname{Tot}\left(I_{\bar{U}}^{\bullet} \rightarrow i_{*} I_{U_{\infty}}^{\bullet}\right) .
$$


Combining these observations gives the isomorphism

$$
\begin{aligned}
\operatorname{Ext}_{h}^{q}\left(z_{\text {equi }}^{0}(-, U)_{h}^{\tilde{h}}, F^{\#}\right) & =H^{q} \operatorname{Hom}_{h}\left(z_{\text {equi }}^{0}(-, U)_{h}^{\sim}, I^{\bullet}\right) \\
& \cong H^{q}\left(\Gamma\left(\bar{U}, \operatorname{Tot}\left(I_{\bar{U}}^{\bullet} \rightarrow i_{*} I_{U_{\infty}}^{\bullet}\right)\right),\right. \\
& =\mathrm{H}_{\text {ét }}^{p}\left(\bar{U}, j_{!}\left(F^{\#}\right)\right) \\
& =\mathrm{H}_{c}^{p}\left(U, F^{\#}\right) .
\end{aligned}
$$

Finally, using [24, 7.6], we obtain

$$
\begin{aligned}
\mathrm{H}_{c}^{p}\left(U, F^{\#}\right) \cong \operatorname{Ext}_{h}^{p}\left(z_{\text {equi }}^{0}(-, U)_{h}^{\sim}, F^{\#}\right) & \cong \operatorname{Ext}_{A b}^{p}\left(z_{\text {equi }}^{0}\left(\Delta_{\text {top }}^{\bullet}, U\right), F^{\#}\right) \\
& \cong \pi_{p}\left(z_{\text {equi }}^{0}\left(\Delta^{\bullet}, U\right) \otimes F\right)^{\#},
\end{aligned}
$$

where the last isomorphism holds since $z_{\text {equi }}^{0}\left(\Delta^{\bullet}, U\right)$ is a complex of free abelian groups. Since $\mathrm{H}_{c}^{p}\left(X, F^{\#}\right)$ is a finitely generated $\mathbb{Z} / n$-module, it follows that $\pi_{p}\left(z_{\text {equi }}^{0}\left(\Delta^{\bullet}, U\right) \otimes F\right)^{\#}$ and hence $\pi_{p}\left(z_{\text {equi }}^{0}\left(\Delta^{\bullet}, U\right) \otimes F\right)$ are finitely generated too, so that applying $(-)^{\#}$ to (21) gives

$$
\mathrm{H}_{c}^{p}\left(X, F^{\#}\right)^{\#} \cong \pi_{p}\left(z_{\text {equi }}^{0}\left(\Delta^{\bullet}, U\right) \otimes F\right)
$$

as desired.

To establish the (contravariant) naturality of (18) with respect to quasi-finite, flat morphisms of normal varieties, it suffices to establish (covariant) naturality of the isomorphism (20) with respect to such morphisms, since the remaining isomorphisms of 21 are clearly natural. Suppose $f: U^{\prime} \rightarrow U$ is a quasi-finite, flat morphism of normal, connected varieties. Choose a projective closure of $U$ and take its normalization, so that we have an open, dense embedding $U \longmapsto \bar{U}$ with $\bar{U}$ normal and projective. By Zariski's Main Theorem, the quasi-finite map $U^{\prime} \rightarrow \bar{U}$ factors as an open embedding $U^{\prime} \longmapsto V$ followed by a finite morphism $V \rightarrow \bar{U}$. Letting $\bar{U}^{\prime}$ be the normalization of $V$, we obtain a commutative square

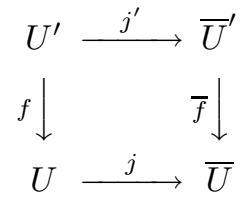

whose horizontal arrows $j, j^{\prime}$ are open immersions into normal, projective varieties and such that the right-hand vertical arrow $\bar{f}$ is finite. Let $i: U_{\infty} \mapsto \bar{U}$ and $i^{\prime}: U_{\infty}^{\prime} \longmapsto \bar{U}^{\prime}$ be the reduced, closed complements of $U$ in $\bar{U}$ and $U^{\prime}$ in $\bar{U}^{\prime}$.

With this set up, the trace map in cohomology with compact supports of the constant sheaf $F^{\#}$ is given as follows. Choose an injective resolution $j_{!}^{\prime} F^{\#} \rightarrow J^{\bullet}$ of sheaves on $\bar{U}_{e t t}^{\prime}$. Then the canonical map $\bar{f}_{*} j_{!}^{\prime} F^{\#} \rightarrow \bar{f}_{*}\left(J^{\bullet}\right)$ is an injective resolution of $\bar{f}_{*} j_{!}^{\prime} F^{\#}$ of sheaves on $\bar{U}_{e t t}$. There is a natural isomorphism $\bar{f}_{*} j_{!}^{\prime} F^{\#} \cong$ $j_{!} f_{!} F^{\#}$, where $f_{!}$is the functor defined in [1, XVII.6]. The trace map is a natural transformation

$$
\operatorname{Tr}_{f}: f_{!} F^{\#} \rightarrow F^{\#}
$$

uniquely characterized by the properties of [1. XVII.6.2.3]. (Note that we identify the constant sheaf $F^{\#}=F_{U^{\prime}}^{\#}$ on $U_{e ́ t}^{\prime}$ with $f^{*}\left(F_{U}^{\#}\right)$.) Applying $j_{\text {! }}$ to $\operatorname{Tr}_{f}$ and using the natural isomorphism above, we obtain a map $\operatorname{Tr}: \bar{f}_{*} j_{!}^{\prime} F^{\#} \rightarrow j_{!} F^{\#}$. Now choose 
an injective resolution $j_{!} F^{\#} \rightarrow I^{\bullet}$, and form the commutative diagram

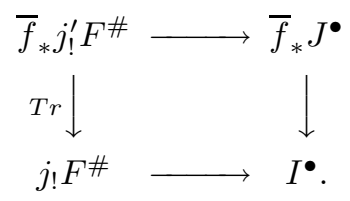

Here, the right-hand vertical map is unique up to homotopy, and upon applying $\Gamma(\bar{U},-)$ to it and taking cohomology groups, one obtains the trace map in cohomology with compact supports

$$
\operatorname{Tr}_{f}: \mathrm{H}_{c}^{*}\left(U^{\prime}, F^{\#}\right)=H^{*} \Gamma\left(\bar{U}^{\prime}, J^{\bullet}\right) \cong H^{*} \Gamma\left(\bar{U}, \bar{f}_{*} J^{\bullet}\right) \rightarrow H^{*} \Gamma\left(\bar{U}, I^{\bullet}\right) \cong \mathrm{H}_{c}^{*}\left(U, F^{\#}\right) .
$$

To complete the proof of naturality, we need a general framework for transfer type maps arising from $q f h$ sheaves. For brevity, throughout the remainder of this proof, for any variety $Z$ we write $c^{0}(Z)$ and $z^{0}(Z)$ for the $q f h$ sheaves of $\mathbb{Z}[1 / p]$ modules $c_{\text {equi }}^{0}(-, Z)[1 / p]$ and $z_{\text {equi }}^{0}(-, Z)[1 / p]$, respectively. Note that there is a natural isomorphism $c^{0}(Z) \cong \mathbb{Z}[1 / p]_{q f h}(Z)=\mathbb{Z}[1 / p] \operatorname{Hom}(-, Z)_{q f h}^{\sim}$ by [25, 4.2.7 and 4.2.12].

Given a closed subscheme $i: Z \longmapsto X$, we may consider the quotient $q f h$ sheaf $c^{0}(X) / c^{0}(Z)$ given as the cokernel of the inclusion $c^{0}(Z) \longmapsto c^{0}(X)$. For any $q f h$ sheaf of $\mathbb{Z}[1 / p]$-modules $\mathcal{G}$, we have

$$
\operatorname{Hom}_{q f h}\left(c^{0}(X) / c^{0}(Z), \mathcal{G}\right)=\operatorname{ker}\left(i^{*}: \Gamma(X, \mathcal{G}) \rightarrow \Gamma(Z, \mathcal{G})\right)
$$

Pulling back cycles along the complementary open subscheme $j: U \subset X$ defines a map

$$
j^{*}: c^{0}(X) / c^{0}(Z) \rightarrow z^{0}(U)
$$

of $q f h$ sheaves. (This map becomes an isomorphism upon $h$ sheafification if $X$ is projective by [25, 4.2.9 and 4.3.1].)

Now suppose $g: X^{\prime} \rightarrow X$ is a morphism such that $X^{\prime}$ is a disjoint union of integral varieties, $X$ is a disjoint union of normal varieties, and each connected component of $X^{\prime}$ maps finitely and dominantly onto a connected component of $X$. Suppose also that $i^{\prime}: Z^{\prime} \longmapsto X^{\prime}$ is a closed subscheme such that $g^{-1}(Z) \subset Z^{\prime}$ and such that the induced map on open complements

$$
f: U^{\prime}=X^{\prime}-Z^{\prime} \stackrel{g}{\longrightarrow} X-Z=U
$$

is flat. Since each component of $X$ is normal, the graph of $g$ defines an element of $c_{\text {equi }}^{0}\left(X, X^{\prime}\right)$, which in light of the natural isomorphisms

$$
\operatorname{Hom}_{q f h}\left(c^{0}(X), c^{0}\left(X^{\prime}\right)\right) \cong \operatorname{Hom}_{q f h}\left(\mathbb{Z}[1 / p](X), c^{0}\left(X^{\prime}\right)\right) \cong c_{\text {equi }}^{0}\left(X, X^{\prime}\right)[1 / p]
$$

defines a map $g^{*}: c^{0}(X) \rightarrow c^{0}\left(X^{\prime}\right)$ of $q f h$ sheaves. (If $g$ happens to be flat, the map $g^{*}$ is given on normal varieties by pullback of cycles.) Moreover, the composition $c^{0}(Z) \longmapsto c^{0}(X) \stackrel{g^{*}}{\longrightarrow} c^{0}\left(X^{\prime}\right)$ factors through $c^{0}\left(Z^{\prime}\right)$ and hence we obtain a map $g^{*}: c^{0}(X) / c^{0}(Z) \rightarrow c^{0}\left(X^{\prime}\right) / c^{0}\left(Z^{\prime}\right)$ of $q f h$ sheaves. Since $f: U^{\prime} \rightarrow U$ is assumed to be flat, we have a commutative diagram of $q f h$ sheaves of the form

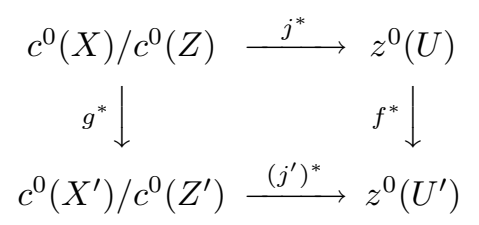


where $j^{\prime}$ denotes the open immersion $U^{\prime} \longmapsto X^{\prime}$ and $f^{*}$ is given by flat pullback of cycles. Consequently, for any $q f h$ sheaf of $\mathbb{Z}[1 / p]$-modules, we have a commutative digram of the form

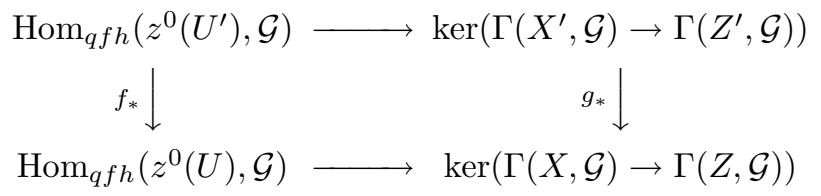

Let $\alpha: V \rightarrow X$ be an étale map, and write $Z_{V}=V \times_{X} Z, X_{V}^{\prime}=V \times_{X} X^{\prime}$, etc. We claim that pushforward along $\alpha$ (and the evident maps induced by $\alpha$ ) defines a commutative square of $q f h$ sheaves

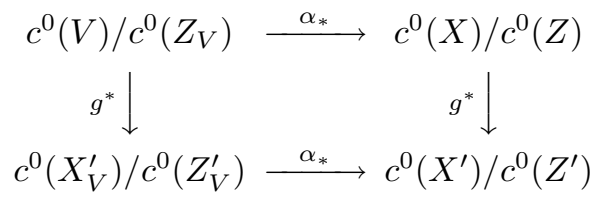

To see this, it suffices to observe that the pullback of the graph of $g$ along $\alpha$ (which gives an element of $c_{e q u i}^{0}\left(V, X^{\prime}\right)$ ) coincides with the image of the graph of the canonical map $X_{V}^{\prime} \rightarrow V$ (which is an element of $c_{e q u i}^{0}\left(V, X_{V}^{\prime}\right)$ ) along the map $X_{V}^{\prime} \rightarrow X^{\prime}$.

If $\mathcal{G}$ is any $q f h$ sheaf of $\mathbb{Z}[1 / p]$-modules, applying $\operatorname{Hom}_{q f h}(-, \mathcal{G})$ to the top arrow of the square (24) for each étale morphism $V \rightarrow X$, we see that the assignment

$$
(V \rightarrow X) \mapsto \operatorname{Hom}_{q f h}\left(c^{0}(V) / c^{0}\left(Z_{V}\right), \mathcal{G}\right)=\operatorname{ker}\left(\Gamma(V, \mathcal{G}) \rightarrow \Gamma\left(Z_{V}, \mathcal{G}\right)\right)
$$

defines an étale sheaf on the small cite $X_{\text {ét }}$ - let us write this sheaf as $\mathcal{G}_{X, Z}$. Note that $\mathcal{G}_{X, Z}$ is merely the étale sheaf $\operatorname{ker}\left(\mathcal{G}_{X} \rightarrow i_{*} \mathcal{G}_{Z}\right)$ defined on $X_{\text {ét }}$. However, this description of the sheaf gives the existence of the transfer map we seek - namely, since the square (24) commutes, the maps

$$
g_{*}: \operatorname{Hom}_{q f h}\left(c^{0}\left(X_{V}^{\prime}\right) / c^{0}\left(Z_{V}^{\prime}\right), \mathcal{G}\right) \rightarrow \operatorname{Hom}_{q f h}\left(c^{0}(V) / c^{0}\left(Z_{V}\right), \mathcal{G}\right)
$$

induced by $g^{*}$ determine a "transfer" morphism of étale sheaves on $X_{e ́ t}$ of the form

$$
g_{*}: g_{*} \mathcal{G}_{X^{\prime}, Z^{\prime}} \rightarrow \mathcal{G}_{X, Z}
$$

Observe that since $f: U^{\prime} \rightarrow U$ is flat, for any étale map $V \rightarrow X$, the commutativity of (23) gives the commutative diagram

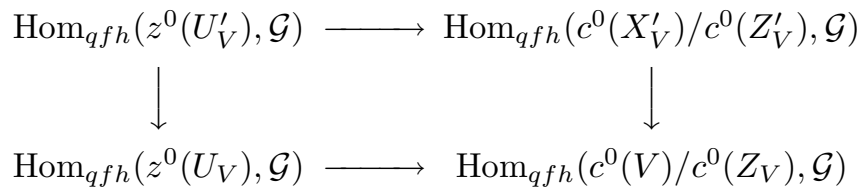

whose left-hand vertical arrow is induced by a flat pullback of cycles. (It is perhaps worth noting that the assignment sending $V \rightarrow X$ to $\operatorname{Hom}_{q f h}\left(z^{0}\left(U_{V}\right), \mathcal{G}\right)$ does not obviously define an étale sheaf, since there is not a reasonable map $z^{0}\left(V_{U}\right) \rightarrow z^{0}(U)$ unless $V_{U} \rightarrow U$ is finite. In particular, we are not asserting that the rows of (25) define morphisms of étale sheaves.)

Now observe that the map $\mathcal{G}_{X} \rightarrow i_{*} \mathcal{G}_{Z}$ factors as $\mathcal{G}_{X} \rightarrow i_{*} i^{*} \mathcal{G}_{X} \rightarrow i_{*} \mathcal{G}_{X}$, and hence there are natural maps

$$
j_{!} \mathcal{G}_{U} \rightarrow \mathcal{G}_{X, Z} \quad \text { and } \quad j_{!}^{\prime} \mathcal{G}_{U^{\prime}} \rightarrow \mathcal{G}_{X^{\prime}, Z^{\prime}},
$$


since $j_{!} \mathcal{G}_{U}=j_{!} j^{*} \mathcal{G}_{X}$ is the kernel of $\mathcal{G}_{X} \rightarrow i_{*} i^{*} \mathcal{G}_{X}$ and similarly for $j_{!}^{\prime} \mathcal{G}_{U^{\prime}}$. Using the canonical map $f^{*} \mathcal{G}_{U} \rightarrow \mathcal{G}_{U^{\prime}}$, we let $j_{!}^{\prime} f^{*} \mathcal{G}_{U} \rightarrow \mathcal{G}_{X^{\prime}, Z^{\prime}}$ be the composition of

$$
j_{!}^{\prime} f^{*} \mathcal{G}_{U^{\prime}} \rightarrow j_{!}^{\prime} \mathcal{G}_{U^{\prime}} \rightarrow \mathcal{G}_{X^{\prime}, Z^{\prime}}
$$

Lemma 7.4. In the situation above, the diagram

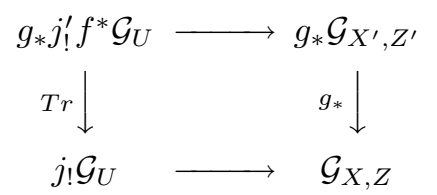

commutes, where $\operatorname{Tr}$ is defined as the composition of

$$
g_{*} j_{!}^{\prime} f^{*} \mathcal{G}_{U} \cong j_{!} f_{!} f^{*} \mathcal{G}_{U} \stackrel{\stackrel{\left(T r_{f}\right)}{\longrightarrow}}{\longrightarrow} j_{!} \mathcal{G}_{U} .
$$

Proof. Since $\mathcal{G}_{X, Z}$ and $\mathcal{G}_{X^{\prime}, Z^{\prime}}$ map to $\mathcal{G}_{X}$ and $\mathcal{G}_{X^{\prime}}$ invectively via maps commuting with $g_{*}$, it suffices to prove

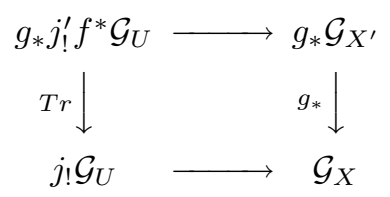

commutes. Moreover, since (26) is a diagram of étale sheaves on $X_{\text {ét }}$ and the sheaves on the left are supported on $U \subset X_{e ́ t}$, it suffices to establish that this diagram commutes at the stalk of an arbitrary point of $U$. Let $U_{u}$ denote the spectrum of the strict henselization of $U$ at a point $u \in U$. Then since $g$ is finite, the scheme $U_{u} \times_{X} X^{\prime}$ decomposes as $V_{0} \amalg V_{1} \amalg \cdots \amalg V_{t}$, such that for each $1 \leq i \leq t$ we have (1) the scheme $V_{i}$ is the spectrum of a strictly hensel local ring, (2) the map $V_{i} \rightarrow U_{u}$ is finite and flat, and (3) the scheme $V_{i}$ contained in the open subscheme $U_{u} \times_{U} U^{\prime}$. Moreover, we have that the closed points of the scheme $V_{0}$ (which is a product of spectra of local rings) are not contained in $U_{u} \times_{U} U^{\prime}$. Noting that $g_{*} j_{!}^{\prime} f^{*} \mathcal{G}_{U} \cong j_{!} f_{!} f^{*} \mathcal{G}_{U}$, applying $\Gamma\left(U_{u},-\right)$ to (26) (i.e., taking stalks at $u$ ) gives the diagram

$$
\begin{array}{ccc}
\Gamma\left(U_{u}, g_{*} j_{!}^{\prime} f^{*} \mathcal{G}_{U}\right)=\bigoplus_{i=1}^{t} \Gamma\left(V_{i}, f^{*} \mathcal{G}_{U}\right) & & \Gamma\left(U_{u}, g_{*} \mathcal{G}_{X^{\prime}}\right)=\bigoplus_{i=0}^{t} \Gamma\left(V_{i}, \mathcal{G}\right) \\
\operatorname{Tr} \downarrow & g_{*} \downarrow \\
\Gamma\left(U_{u}, \mathcal{G}\right) & \longrightarrow & \Gamma\left(U_{u}, \mathcal{G}\right),
\end{array}
$$

where the top horizontal arrow is a composition of the canonical map and the evident summand inclusion:

$$
\bigoplus_{i=1}^{t} \Gamma\left(V_{i}, f^{*} \mathcal{G}_{U}\right) \rightarrow \bigoplus_{i=1}^{t} \Gamma\left(V_{i}, \mathcal{G}_{U}\right) \longmapsto \bigoplus_{i=0}^{t} \Gamma\left(V_{i}, \mathcal{G}_{U}\right) .
$$

Moreover, for each $i=1, \ldots, t$, since $U_{u}$ is the spectrum of a strictly henselian ring, the natural map $\Gamma\left(U_{u}, \mathcal{G}_{U}\right) \rightarrow \Gamma\left(V_{i}, f^{*} \mathcal{G}_{U}\right)$ is an isomorphism, and the composition of

$$
\Gamma\left(U_{u}, \mathcal{G}\right) \stackrel{\cong}{\longrightarrow} \Gamma\left(V_{i}, f_{*} \mathcal{G}_{U}\right) \stackrel{T r}{\longrightarrow} \Gamma\left(U_{u}, \mathcal{G}\right)
$$

is multiplication by the degree of the finite, flat map $V_{i} \rightarrow U_{u}$ (cf. [1, XVII.6.2.3]). It therefore suffices to prove that for $i=1, \ldots, t$, the composition of

$$
\Gamma\left(U_{u}, \mathcal{G}\right) \stackrel{g^{*}}{\longrightarrow} \Gamma\left(V_{i}, \mathcal{G}\right) \stackrel{g_{*}}{\longrightarrow} \Gamma\left(U_{u}, \mathcal{G}\right)
$$


is also multiplication by the degree of $V_{i} \rightarrow U_{u}$. Since $V_{i} \rightarrow U_{u}$ is an inverse limit of finite, flat maps of normal varieties, it suffices to prove that the composition of

$$
\Gamma(S, \mathcal{G}) \stackrel{h^{*}}{\longrightarrow} \Gamma(T, \mathcal{G}) \stackrel{h_{*}}{\longrightarrow} \Gamma(S, \mathcal{G})
$$

is multiplication by $\operatorname{deg}(h)$ for any finite, flat morphism $h: T \rightarrow S$ of normal varieties. This is turn holds since the composition of

$$
c^{0}(S) \stackrel{g^{*}}{\longrightarrow} c^{0}(T) \stackrel{g_{*}}{\longrightarrow} c^{0}(S)
$$

is given by composing pullback and pushforward along the flat morphism $h$, and is thus multiplication by $\operatorname{deg}(h)$.

We now return to the main argument of the proof of Theorem 7.3 The square (22) satisfies the needed hypotheses so that, by Lemma 7.4, given any $q f h$ sheaf of $\mathbb{Z}[1 / p]$-modules, we have a commutative square

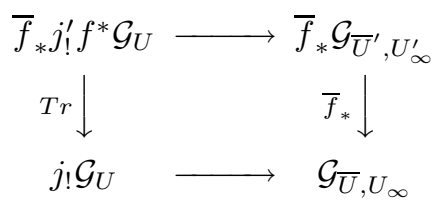

of $q f h$ sheaves. In particular, we apply this to each sheaf in the injective resolution $F^{\#} \rightarrow I^{\bullet}$ consisting of $h$-sheaves of $\mathbb{Z}[1 / p]$-modules to obtain a commutative diagram

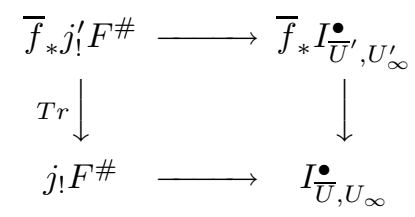

of complexes of étale sheaves on $\bar{U}_{e t}$. (Note that $f^{*} F_{U}^{\#}=F_{U^{\prime}}^{\#}=F^{\#}$, since $F^{\#}$ is a constant sheaf.) Since $I_{\bar{U}, U_{\infty}}^{\bullet}$ coincides with the total complex of $I_{\bar{U}}^{\bullet} \rightarrow I_{U_{\infty}}^{\bullet}$ (and similarly for $I_{\bar{U}^{\prime}, U_{\infty}^{\prime}}^{\bullet}$ ), the rows of this diagram represent injective resolutions of $\bar{f}_{*} j_{!}^{\prime} F^{\#}$ and $j_{!} F^{\#}$. Thus, from our description given earlier, applying $\Gamma(\bar{U},-)$ to the right-hand column of this diagram and taking cohomology gives the trace map in étale cohomology with compact supports. On the other hand, the diagram

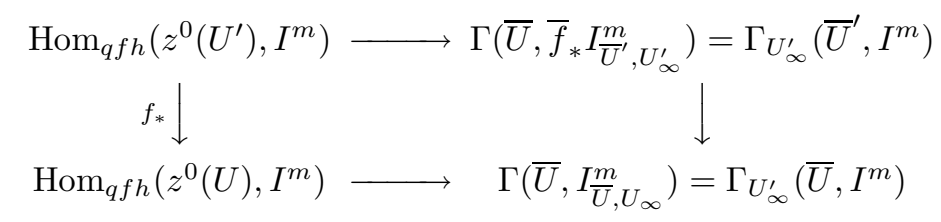

commutes for each $m$ by (25), from which it follows that the isomorphism (20) is natural as claimed. (Note that $\operatorname{Hom}_{q f h}\left(z^{0}(Y), \mathcal{G}\right)$ is naturally isomorphic to $\operatorname{Hom}_{h}\left(z^{0}(Y)_{h}, \mathcal{G}\right)$ if $\mathcal{G}$ is an $h$ sheaf of $\mathbb{Z}[1 / p]$-modules.) The isomorphism (18) is therefore natural for quasi-finite, flat morphisms of normal varieties.

Finally, if $U$ is smooth of pure dimension $d$, then Poincaré duality for étale cohomology (cf. [1, XVIII.3.2.5]) gives the isomorphism

$$
\mathrm{H}_{c}^{p}\left(U, \mu_{n}^{\otimes m / 2}(k)^{\#}\right)^{\#} \cong \mathrm{H}_{\text {ét }}^{2 d-p}\left(U, \mu_{n}^{\otimes(d+m / 2)}\right) .
$$


The naturality of this isomorphism with respect to a quasi-finite, flat morphism follows from the functoriality and compatibility with composition of the trace map as shown in [1, XVIII.2.9].

The following theorem relates $K$-groups with quasi-finite support to the presheaves $z_{\text {equi }}^{0}(-, U)$ and hence to étale cohomology.

Theorem 7.5. Let $U$ be a Cohen-Macaulay quasi-projective variety defined over an algebraically closed field $k$ and assume $n \geq 1$ is an integer relatively prime to the exponential characteristic of $k$. Then there is a homotopy equivalence of simplicial abelian groups

$$
\left(d \mapsto z_{\text {equi }}^{0}\left(\Delta^{d}, U\right) \otimes_{\mathbb{Z}} \mu_{n}^{\otimes m / 2}(k)\right) \sim\left(d \mapsto K_{m}^{q f}\left(\Delta^{d}, U ; \mathbb{Z} / n\right)\right),
$$

where $\mu_{n}^{\otimes m / 2}(k)$ is defined to be the zero group for $m$ odd. Consequently, we have an isomorphism

$$
\pi_{p}\left(d \mapsto K_{m}^{q f}\left(\Delta^{d}, U ; \mathbb{Z} / n\right)\right) \cong \mathrm{H}_{c}^{p}\left(U, \mu_{n}^{\otimes m / 2}(k)^{\#}\right)^{\#},
$$

which is contravariantly natural with respect to quasi-finite, flat morphisms of normal, Cohen-Macaulay varieties.

Moreover, if $U$ is smooth of pure dimension d, we have in addition the isomorphism

$$
\pi_{p}\left(d \mapsto K_{m}^{q f}\left(\Delta^{d}, U ; \mathbb{Z} / n\right)\right) \cong \mathrm{H}_{e ́ t}^{2 d-p}\left(U, \mu_{n}^{\otimes(d+m / 2)}\right),
$$

which is contravariantly natural with respect to quasi-finite, flat morphisms of smooth varieties.

Proof. Theorem 6.2 and Proposition 5.2 imply that the $h$ sheafification of $K_{0}^{q f}(-, U)$ is isomorphic to $z_{\text {equi }}^{0}(-, U)$. It follows from Corollary 4.23 that the cycle class map

$$
K_{0}^{q f}\left(\Delta^{\bullet}, U ; \mathbb{Z} / n\right) \stackrel{\sim}{\longrightarrow} z_{\text {equi }}^{0}\left(\Delta^{\bullet}, U ; \mathbb{Z} / n\right)
$$

defines a homotopy equivalence of simplicial abelian groups. (The map $K_{0}^{q f}(-, U)$ $\rightarrow z_{\text {equi }}^{0}(-, U)$ is readily verified to be a morphism of presheaves with $K_{0}$ transfers.) Applying Theorem 4.24 and Corollary 4.23 to the map

$$
K_{0}^{q f}(-, U) \otimes K_{m}(k ; \mathbb{Z} / n) \rightarrow K_{m}^{q f}(-, U ; \mathbb{Z} / n)
$$

and using Theorem 7.1, we obtain homotopy equivalences

$$
z_{\text {equi }}^{0}\left(\Delta^{\bullet}, U\right) \otimes K_{m}(k ; \mathbb{Z} / n) \sim K_{0}^{q f}\left(\Delta^{\bullet}, U\right) \otimes K_{m}(k ; \mathbb{Z} / n) \sim K_{m}^{q f}\left(\Delta^{\bullet}, U ; \mathbb{Z} / n\right)
$$

of simplicial abelian groups. (The map (27) is a morphism of presheaves with $K_{0}$ transfers since the two evident maps of the form

$$
\mathcal{K}^{f i n}(X / S) \wedge \mathcal{K}^{q f}(X, U) \wedge \mathcal{K}(k) \rightarrow \mathcal{K}^{q f}(S, U)
$$

coincide up to homotopy.) The cup product operation in $K$-theory defines a natural map

$$
\mu_{n}(k)^{\otimes t} \rightarrow K_{2 t}(k ; \mathbb{Z} / n),
$$

which by Suslin's theorem [23 3.13] is an isomorphism. Moreover, Suslin's theorem also shows that $K_{2 t+1}(k ; \mathbb{Z} / n)=0$.

The remaining assertions follow directly from Theorem 7.3 
In Section 5 we used $\mathcal{K}^{q f}(X, U ; \mathbb{Z} / n)$ to denote the spectrum obtained from $\mathcal{K}^{q f}(X, U)$ by smashing with the mod-n Moore spectrum. For the purposes of this section, we need a more explicit and functorial model for the space whose homotopy groups are $K_{*}^{q f}(X, U ; \mathbb{Z} / n)$. Specifically, let $M=M_{n}^{2}$ denote the singular simplicial set associated to the mapping cone of $S^{1} \stackrel{\cdot n}{\longrightarrow} S^{1}$ and let $\Omega^{-2} \mathcal{K}^{q f}(-, U)$ denote a functor taking values in Kan complexes that gives a functorial 2-fold delooping of $\mathcal{K}^{q f}(-, U)$. Namely, we define

$$
\Omega^{-2} \mathcal{K}^{q f}(-, U)=\operatorname{Ex}\left(\operatorname{diag}\left(S \bullet S \cdot \mathcal{P}^{q f}(-, U)\right)\right)
$$

where $S_{\bullet}$ is the Waldhausen $S$ construction (which may be iterated to form a bisimplicial set as above), diag denotes taking the diagonal of a bisimplicial set, and Ex is the Kan extension functor taking simplicial sets to Kan complexes. (Recall also that $\mathcal{P}^{q f}(-, U)$ is made strictly functorial as discussed in Section [3.) Let $\underline{\text { Maps }}_{*}(-,-)$ denote the simplicial mapping space functor. Then the geometric

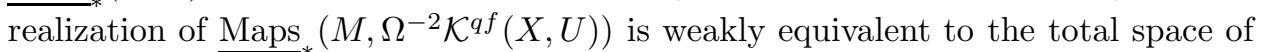
the spectrum we wrote as $\mathcal{K}^{q f}(X, U ; \mathbb{Z} / n)$ before, so that we have

$$
\pi_{t} \underline{\operatorname{Maps}}_{*}\left(M, \Omega^{-2} \mathcal{K}^{q f}(X, U)\right) \cong K_{t}^{q f}(X, U ; \mathbb{Z} / n),
$$

for all $t \geq 0$. Indeed, in the remainder of this section, we will write $\mathcal{K}^{q f}(X, U ; \mathbb{Z} / n)$ for the Kan complex $\operatorname{Maps}_{*}\left(M, \Omega^{-2} \mathcal{K}^{q f}(X, U)\right)$. In particular, $\mathcal{K}^{q f}(X, Y ; \mathbb{Z} / n)$ is

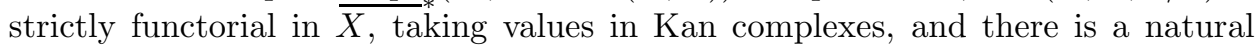
isomorphism

$$
\pi_{q} \mathcal{K}^{q f}(X, Y ; \mathbb{Z} / n) \cong K_{q}^{q f}(X, Y ; \mathbb{Z} / n) .
$$

Theorem 7.6. Let $U$ be a Cohen-Macaulay, quasi-projective variety defined over an algebraically closed field $k$ and assume $n \geq 1$ is an integer relatively prime to the exponential characteristic of $k$. Then there is a convergent spectral sequence

$$
E_{s, t}^{2}=\mathrm{H}_{c}^{s}\left(U, \mu_{n}^{\otimes t / 2}(k)^{\#}\right)^{\#} \Longrightarrow K_{s+t}^{B M}(U ; \mathbb{Z} / n),
$$

where $\mu_{n}^{\otimes t / 2}(k)=0$ for $t$ odd. Here, the indexing is homological (so that the $d_{2}$ differential has bidegree $(-2,1)$ ) and the $E_{2}$ terms are concentrated in the portion of the first quadrant of the st-plane with $s \leq 2 \operatorname{dim}(U)$. Moreover, the spectral sequence is natural with respect to all quasi-finite, flat morphisms of normal, CohenMacaulay varieties.

When $U$ is smooth, we may write this as a cohomologically indexed convergent spectral sequence (with $d_{2}$ differential having bidegree $(2,-1)$ )

$$
E_{2}^{p, q}=\mathrm{H}_{\text {ét }}^{p-q}\left(U, \mu_{n}^{\otimes(-q)}(k)\right) \Longrightarrow K_{-p-q}^{B M}(U ; \mathbb{Z} / n)
$$

which is concentrated in the portion of the pq-plane defined by $p \geq q \geq p-2 d$ and $q \leq-d$ and which is natural for quasi-finite, flat morphisms of smooth varieties.

Proof. The first spectral sequence is the standard spectral sequence associated to the simplicial space $d \mapsto \mathcal{K}^{q f}\left(\Delta^{d}, U ; \mathbb{Z} / n\right)$. Since we will need the details of this construction later, we recall them here.

If $Y=\left(d \mapsto Y_{d}\right)$ is a pointed Kan complex, let $P_{t}(Y), t \geq 0$, denote the Postnikov truncation of $Y$, so that $P_{t}(Y)$ is a quotient of $Y$ defined by the equivalence relation where two $n$-simplices of $Y$ are equivalent if their $t$-skeleta coincide. The natural 
surjection $Y \rightarrow P_{t}(Y)$ induces an isomorphism on $\pi_{n}$ for $n \leq t$, and $\pi_{n} P^{t}(Y)=0$ for $n>t$. Define $Q_{t}(Y)$ to be the fiber of the Kan fibration

$$
P_{t}(Y) \rightarrow P_{t-1}(Y)
$$

so that $Q_{t}(Y)$ is a Eilenberg-Mac Lane Kan complex whose only non-vanishing homotopy group is $\pi_{t} Q_{t}(Y) \cong \pi_{t}(Y)$. Applying $P_{t}(-)$ to the Kan complexes $\mathcal{K}^{q f}\left(\Delta^{d}, U ; \mathbb{Z} / n\right)$, for $d \geq 0$, and then taking diagonals gives a tower of fibration sequences

$$
\begin{aligned}
\cdots \rightarrow \operatorname{diag}(d & \left.\mapsto P_{t+1} \mathcal{K}^{q f}\left(\Delta^{d}, U ; \mathbb{Z} / n\right)\right) \rightarrow \operatorname{diag}\left(d \mapsto P_{t} \mathcal{K}^{q f}\left(\Delta^{d}, U ; \mathbb{Z} / n\right)\right) \\
& \rightarrow \cdots \rightarrow \operatorname{diag}\left(d \mapsto P^{0} \mathcal{K}^{q f}\left(\Delta^{d}, U ; \mathbb{Z} / n\right)\right) \rightarrow *
\end{aligned}
$$

such that the fiber of the $t$-th map is $\operatorname{diag}\left(d \mapsto Q_{t} \mathcal{K}^{q f}\left(\Delta^{d}, U ; \mathbb{Z} / n\right)\right)$. The definitions

$$
D_{s, t}^{2}=\pi_{s+t+1}\left|d \mapsto P_{t-1} \mathcal{K}^{q f}\left(\Delta^{d}, U ; \mathbb{Z} / n\right)\right|
$$

and

$$
E_{s, t}^{2}=\pi_{s+t}\left|d \mapsto Q_{t} \mathcal{K}^{q f}\left(\Delta^{d}, U ; \mathbb{Z} / n\right)\right| \cong \pi_{s}\left|d \mapsto K_{t}^{q f}\left(\Delta^{d}, U ; \mathbb{Z} / n\right)\right|
$$

and the maps appearing in the long exact sequences associated to these fibration sequences determine an exact couple and thus a spectral sequence abutting to $\pi_{*}\left|d \mapsto \mathcal{K}^{q f}\left(\Delta^{d}, U ; \mathbb{Z} / n\right)\right|$. Theorem [7.5] shows that the $E^{2}$ terms are isomorphic to the asserted étale cohomology groups. Finally, to show that the abutment of this spectral sequence is isomorphic to $K_{*}^{\mathrm{BM}}(U ; \mathbb{Z} / n)$, we observe that we have the fibration sequence

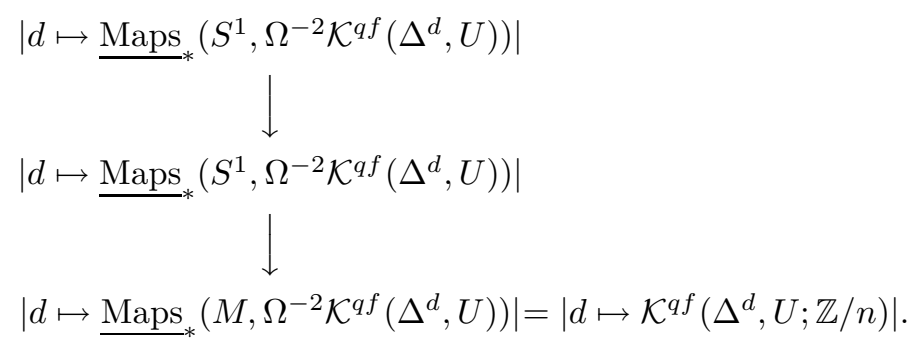

Moreover, using [30, 5.2], we have homotopy equivalences

$$
\begin{aligned}
\Omega\left|d \mapsto \underline{\operatorname{Maps}}_{*}\left(S^{1}, \Omega^{-2} \mathcal{K}^{q f}\left(\Delta^{d}, U\right)\right)\right| & \sim\left|d \mapsto \underline{\operatorname{Maps}}_{*}\left(S^{2}, \Omega^{-2} \mathcal{K}^{q f}\left(\Delta^{d}, U\right)\right)\right| \\
& \sim \mathcal{K}^{B M}(U),
\end{aligned}
$$

and it follows from the five lemma that we have

$$
\pi_{*}\left|d \mapsto \mathcal{K}^{q f}\left(\Delta^{d}, U ; \mathbb{Z} / n\right)\right| \cong K_{*}^{\mathrm{BM}}(U ; \mathbb{Z} / n) .
$$

(That is, to attain $\mathcal{K}^{B M}(Y ; \mathbb{Z} / n)$ up to weak homotopy, we may apply $\underline{\operatorname{Maps}}_{*}(M,-)$ degree-wise.)

Thus the first spectral sequence exists. The inequality of $s \leq 2 \operatorname{dim}(U)$ holds since $U$ has étale cohomological dimension of at most $2 d$. This bound also establishes the convergence of the spectral sequence. The naturality assertion follows from the contravariant naturality for quasi-finite morphisms of $\Omega^{-2} \mathcal{K}^{q f}(X,-)$ and the naturality assertion of Theorem 7.5 .

The second spectral sequence is constructed by using Poincaré duality for étale cohomology and re-indexing the first spectral sequence. That is, if $U$ is smooth of pure dimension $d$, then we have the isomorphism

$$
\mathrm{H}_{c}^{p}\left(U, \mu_{n}^{\otimes q / 2}(k)^{\#}\right)^{\#} \cong \mathrm{H}_{\text {ét }}^{2 d-p}\left(U, \mu_{n}^{\otimes(d+q / 2)}(k)\right),
$$


which is natural with respect to quasi-finite, flat morphisms. Now perform the linear change of indexing $q=-d-t / 2$ and $p=d-s-t / 2$ to form a spectral sequence with $E_{2}$ terms $\tilde{E}_{2}^{p, q}$. Note that $\tilde{E}_{2}^{p, q}=0$ for $q$ odd, and so we can progress through the pages of the spectral sequence in steps of two without losing any information. That is, we form a new spectral sequence by writing $E_{r}^{p, q}$ for $\tilde{E}_{2 r-1}^{p, q}$, (and similarly for the $D$ terms), which gives us the desired spectral sequence. The case of a general smooth $U$ follows.

Corollary 7.7. Let $U$ be a Cohen-Macaulay, quasi-projective variety defined over an algebraically closed field $k$ and assume $n \geq 1$ is an integer relatively prime to the exponential characteristic of $k$. Then $K_{m}^{B M}(U ; \mathbb{Z} / n)$ is a finite abelian group for all $m \geq 0$.

Proof. This follows from the theorem and the fact that for any constructible sheaf $F$, the group $\mathrm{H}_{c}^{p}(U ; F)$ is finite and vanishes unless $0 \leq p \leq 2 \operatorname{dim}(U)$ (cf. [19] VI.1.1 and VI.2.1]).

A slight extension of the proofs of Theorems 7.6 and 7.3 gives a bit more than we have asserted - namely, the functor $\mathcal{K}^{B M}(-; \mathbb{Z} / n)$ satisfies "étale descent" in a manner made precise in the following statement. To clarify the terminology of this result, recall that an étale hypercovering of a variety $U$ is an augmented simplicial variety $U_{\bullet} \rightarrow U$ such that the canonical map $U_{n} \rightarrow \operatorname{cosk}_{n-1}^{U}\left(U_{\bullet}\right)_{n}$ is an étale covering for all $n \geq 0$. Here, $\operatorname{cosk}_{n-1}^{U}$ denotes the $(n-1)$-st coskeleton of a simplicial scheme over $U$. For example, given an étale covering $U_{0} \rightarrow U$, its nerve $U_{n}=U_{0} \times_{U} \cdots \times_{U} U_{0}, n \geq 0$, defines an étale hypercovering. A cosimplicial space $\mathcal{X}^{\bullet}$ is a functor from the standard simplicial category $\Delta$ (consisting of ordered sets $[n]=\{0<\cdots<n\}, n \geq 0$, and order preserving functions) to the category of spaces, written $d \mapsto \mathcal{X}^{d}$. Observe that $\mathcal{K}^{B M}(-; \mathbb{Z} / n)$ (or any contravariant functor from varieties to spaces) sends an étale hypercovering $U \bullet \rightarrow U$ to a cosimplicial space $d \mapsto \mathcal{K}^{B M}\left(U_{d} ; \mathbb{Z} / n\right)$ equipped with an augmentation map $\mathcal{K}^{B M}(U ; \mathbb{Z} / n) \rightarrow$ $\mathcal{K}^{B M}\left(U_{0} ; \mathbb{Z} / n\right)$. Finally, the homotopy inverse limit of a cosimplicial space $\mathcal{X} \bullet$, written $\bigsqcup^{h} \mathcal{X}^{\bullet}$, is the space defined by [6] XI.4.4].

Theorem 7.8. Let $U$ be a normal, Cohen-Macaulay, quasi-projective variety defined over an algebraically closed field $k$, let $U \bullet \rightarrow U$ be an étale hypercovering, and assume $n \geq 1$ is an integer relatively prime to the exponential characteristic of $k$. Then the canonical map from $\mathcal{K}^{B M}(U ; \mathbb{Z} / n)$ to the homotopy inverse limit of the cosimplicial space [6, XI.3.2] $d \mapsto \mathcal{K}^{B M}\left(U_{d} ; \mathbb{Z} / n\right)$ is a weak homotopy equivalence:

$$
\mathcal{K}^{B M}(U ; \mathbb{Z} / n) \stackrel{\sim}{\longrightarrow} \underbrace{\operatorname{holim}}_{\Delta} \mathcal{K}^{B M}\left(U_{\bullet} ; \mathbb{Z} / n\right) .
$$

Proof. Summarizing the constructions of the proof of Theorem[7.6, we have a tower of Kan complexes, which we write here as

$$
\cdots \rightarrow G_{t}(V) \rightarrow G_{t-1}(V) \rightarrow \cdots \rightarrow G_{0}(V) \rightarrow *,
$$

together with a family of Kan fibration sequences of the form

$$
F_{t}(V) \rightarrow G_{t}(V) \rightarrow G_{t-1}(V), \quad t \geq 0 .
$$

(By definition, $G_{-1}(V)=*$.) Specifically, $G_{t}(V)=\operatorname{diag}\left(d \mapsto P_{t} \mathcal{K}^{q f}\left(\Delta^{d}, V ; \mathbb{Z} / n\right)\right)$ and $F_{t}(V)=\operatorname{diag}\left(d \mapsto Q_{t} \mathcal{K}^{q f}\left(\Delta^{d}, V ; \mathbb{Z} / n\right)\right)$. Moreover, the canonical map

$$
\mathcal{K}^{B M}(V ; \mathbb{Z} / n) \rightarrow \underset{t}{\lim } G_{t}(V) \sim \underset{t}{\operatorname{holim}} G_{t}(V)
$$


is a homotopy equivalence. All of these maps are natural in $V$ for quasi-finite, flat morphisms. Thus, for all $t \geq 0$, we have a diagram

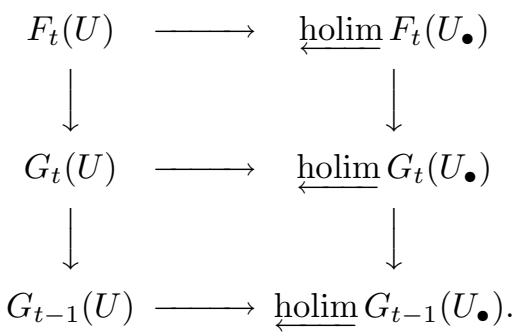

Since for each $U_{i}$ and $t \geq 0$, the map $G_{t}\left(U_{i}\right) \rightarrow G_{t-1}\left(U_{i}\right)$ is a Kan fibration inducing a surjection on $\pi_{0}$, and the right-hand column of this diagram is also a Kan fibration sequence by [6] XI.5.5].

Suppose $F_{t}(U) \rightarrow$ holim $F_{t}\left(U_{\bullet}\right)$ were a homotopy equivalence for all $t \geq 0$. Then using diagram (28) and induction on $t$, we would have that $G_{t}(U) \rightarrow \underset{\operatorname{holim}}{ } G_{t}\left(U_{\bullet}\right)$ is a homotopy equivalence for all $t \geq 0$. Hence, the canonical map

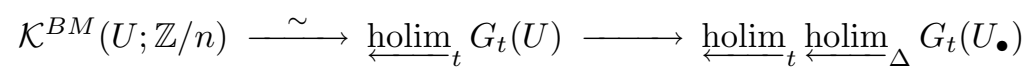

would be a homotopy equivalence. Then by the "Fubini Theorem" for homotopy limits (cf. [6] XI.4.3]), we would obtain a natural homotopy equivalence

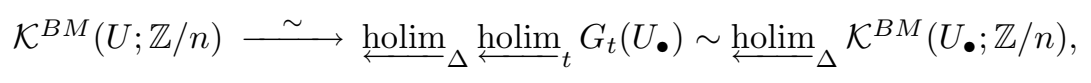

as desired.

It therefore remains to show $F_{t}(U) \rightarrow$ holim $F_{t}\left(U_{\bullet}\right)$ is a homotopy equivalence for all $t \geq 0$. Observe that there is a homotopy equivalence of bisimplicial sets

$$
\left(d \mapsto Q_{t} \mathcal{K}^{q f}\left(\Delta^{d}, U ; \mathbb{Z} / n\right)\right) \rightarrow\left(d \mapsto \Gamma\left(K_{t}^{q f}\left(\Delta^{d}, U ; \mathbb{Z} / n\right), t\right)\right),
$$

where for any abelian group $A$ and integer $t$, we write $\Gamma(A, t)$ for the simplicial abelian group associated to the (homological) chain complex $A[-t]$ consisting of the group $A$ concentrated in homological degree $t$. Moreover, this homotopy equivalence is natural in $U$. Since $F_{t}(U)$ is by definition the diagonal of the first of these bisimplicial sets, it suffices to prove

$$
\operatorname{diag}\left(d \mapsto \Gamma\left(K_{t}^{q f}\left(\Delta^{d}, U ; \mathbb{Z} / n\right), t\right)\right) \rightarrow \underset{\Delta}{\operatorname{holim}} \operatorname{diag}\left(d \mapsto \Gamma\left(K_{t}^{q f}\left(\Delta^{d}, U_{\bullet} ; \mathbb{Z} / n\right), t\right)\right),
$$

which is a map of simplicial abelian groups, is a homotopy equivalence. Every cosimplicial simplicial abelian group $A=A \bullet$ is fibrant [6, X.4.9] and hence $\mathrm{holim}_{\Delta}\left(d \mapsto A_{\bullet}^{d}\right)$ coincides up to a natural homotopy equivalence with the total space Tot $A:$ [6, XI.4.2]. Moreover, the normalized chain complex of $\operatorname{Tot} A \bullet$ (which is a simplicial abelian group) coincides up to a natural quasi-isomorphism with the total complex of the normalized bicomplex $\mathcal{N}^{\bullet} \mathcal{N}_{\bullet} A_{\bullet}$ associated to $A:$ [4] 2.2]. Finally, for any simplicial abelian group $B$ • (such as $d \mapsto K_{q}^{q f}\left(\Delta^{d}, V ; \mathbb{Z} / n\right)$ ), the normalized chain complex associated to $\operatorname{diag}\left(d \mapsto \Gamma\left(B_{d}, t\right)\right)$ coincides up to a natural quasi-isomorphism with $\mathcal{N}_{\bullet}\left(B_{\bullet}\right)[-t]$, where $[-t]$ denotes shifting $t$ degrees to the right. 
Combining these facts, we see that upon taking normalized chain complexes associated to (29), applying Theorem [7.5] and reindexing, it suffices to prove

$$
\mathcal{N}_{\bullet}\left(z_{\text {equi }}^{0}\left(\Delta^{\bullet}, U\right) \otimes F\right) \rightarrow \operatorname{Tot} \mathcal{N}^{\bullet} \mathcal{N}_{\bullet}\left(z_{\text {equi }}^{0}\left(\Delta^{\bullet}, U_{\bullet}\right) \otimes F\right)
$$

is a quasi-isomorphism of chain complexes for any finite $\mathbb{Z} / n$-module $F$. (In this context, Tot refers to taking the total complex associated to a bicomplex.)

We now use the notation and results of the proof of Theorem 7.3. By repeatedly applying Zariski's main theorem and taking normalizations, we can form a augmented "semi-simplicial variety" $\left(d \mapsto X_{d}\right) \rightarrow X$ (i.e., an augmented simplicial variety without degeneracy maps) and a map of augmented semi-simplicial varieties

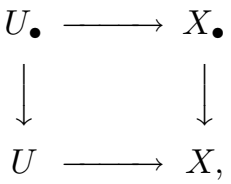

such that (1) $X$ and each $X_{d}, d \geq 0$, are disjoint unions of projective, normal varieties, (2) the maps $U \longmapsto X$ and $U_{d} \longmapsto X_{d}, d \geq 0$ are open, dense immersions, and (3) all of the face maps $X_{d} \rightarrow X_{d-1}, d \geq 1$, and the map $X_{0} \rightarrow X$ send connected components of the source finitely and dominantly onto connected components of the target. Let $j: U \longmapsto X$ and $j_{d}: U_{d} \longmapsto X_{d}, d \geq 0$, denote these open immersions, and let $i: Z \longmapsto X$ and $i_{d}: Z_{d} \longmapsto X_{d}, d \geq 0$, be the complementary closed embeddings. For each $d \geq 0$, let $f_{d}: X_{d} \rightarrow X$ be the unique map induced by the augmentation map. Then, as in the proof of Theorem 7.3, for any $h$ sheaf of $\mathbb{Z}[1 / p]$ modules, we have étale sheaves $\mathcal{G}_{X, Z}$ (defined on $X_{e ́ t}$ ) and $\mathcal{G}_{X_{d}, Z_{d}}$ (defined on $\left.\left(X_{d}\right)_{e t}\right)$, for $d \geq 0$. Moreover, the augmentation map $f_{0}: X_{0} \rightarrow X$ and the various face maps $d_{i}: X_{d} \rightarrow X_{d-1}$ define transfer morphisms of étale sheaves $\left(f_{0}\right)_{*}:\left(f_{0}\right)_{*} \mathcal{G}_{X_{0}, Z_{0}} \rightarrow \mathcal{G}_{X, Z}$ and $\left(d_{i}\right)_{*}:\left(d_{i}\right)_{*} \mathcal{G}_{X_{d}, Z_{d}} \rightarrow \mathcal{G}_{X_{d-1}, Z_{d-1}}$. Upon pushing all of these maps forward to maps of étale sheaves on $X_{e ́ t}$, we obtain a chain complex of sheaves on $X_{e ́ t}$ of the form

$$
\cdots \rightarrow\left(f_{d}\right)_{*} \mathcal{G}_{X_{d}, Z_{d}} \rightarrow \cdots \rightarrow\left(f_{0}\right)_{*} \mathcal{G}_{X_{0}, Z_{0}} \rightarrow \mathcal{G}_{X, Z}
$$

with maps given by taking alternating sums of transfer maps associated to the face maps of the semi-simplicial variety $X_{\bullet}$.

In particular, we apply this construction to each sheaf in an injective resolution $F^{\#} \rightarrow I^{\bullet}$ by $h$ sheaves of $\mathbb{Z}[1 / p]$-modules, to obtain the bicomplex

$$
\cdots \rightarrow\left(f_{d}\right)_{*} I_{X_{d}, Z_{d}}^{\bullet} \rightarrow \cdots \rightarrow\left(f_{0}\right)_{*} I_{X_{0}, Z_{0}}^{\bullet} \rightarrow I_{X, Z}^{\bullet}
$$

of sheaves on $X_{e ́ t}$. The isomorphisms (20) and (21) show that applying $\Gamma(X,-)$ to (31) yields a bicomplex whose total complex is quasi-isomorphic to the complex associated to the map (30), and so it suffices to prove the total complex of (31) is exact.

Using Lemma 7.4, there is a quasi-isomorphism of chain complexes of sheaves from

$$
\cdots \rightarrow\left(f_{d}\right)_{*}\left(j_{d}\right)_{!} F^{\#} \rightarrow \cdots \rightarrow\left(f_{0}\right)_{*}\left(j_{0}\right) ! F^{\#} \rightarrow j_{!} F^{\#}
$$

to (31), where the maps of (32) are given by the alternating sums of trace maps associated to the face maps of the simplicial variety $d \mapsto U_{d}$. (Note that these face maps are étale morphisms.) Thus, it suffices to show (32) is exact.

Let $g_{d}: U_{d} \rightarrow U$ be the unique map induced by the augmentation map. We have $\left(f_{d}\right)_{*}\left(j_{d}\right) ! F^{\#} \cong j_{!}\left(g_{d}\right) ! F^{\#}$, and, in particular, each sheaf appearing in (32) 
is supported on $U$. For an arbitrary point $u$ of $U$, let $U_{u}$ denote the spectrum of the strict henselization of $U$ and $u$, and define $U_{d, u}=U_{d} \times_{U} U_{u}$, for $d \geq 0$. Since each map $g_{d}: U_{d} \rightarrow U$ is étale, the scheme $U_{d, u}$ decomposes as a disjoint union of copies of $U_{u}$ and components whose images in $U_{u}$ do not contain the unique closed point $u$. Let $U_{d, u}^{\prime}$ denote the open and closed subscheme of $U_{d, u}$ consisting of those components that are isomorphic to $U_{u}$. Then, by definition of the maps $\left(g_{d}\right)_{\text {! }}$, we have

$$
\Gamma\left(U_{u},\left(f_{d}\right)_{*}\left(j_{d}\right) ! F^{\#}\right) \cong \Gamma\left(U_{u}, j_{!}\left(g_{d}\right) ! F^{\#}\right) \cong \Gamma\left(U_{u},\left(g_{d}\right) ! F^{\#}\right)=\Gamma\left(U_{d, u}^{\prime}, F^{\#}\right) .
$$

One may readily verify that

$$
\left(d \mapsto U_{d, u}^{\prime}\right) \rightarrow U_{u}
$$

is an augmented simplicial scheme and defines an étale hypercovering of $U_{u}$. Hence the complex

$$
\cdots \rightarrow \Gamma\left(U_{d}^{\prime}, F^{\#}\right) \rightarrow \cdots \rightarrow \Gamma\left(U_{0}^{\prime}, F^{\#}\right) \rightarrow \Gamma\left(U_{u}, F^{\#}\right)
$$

is exact, from which it follows that (32) is exact locally at $u$.

Remark 7.9. Using the general spectral sequence associated to a cosimplicial space described in [6, XI.7.5], we have a spectral sequence whose $E_{2}$ terms are given by

$$
E_{2}^{p, q}=\check{\mathrm{H}}_{\text {ét }}^{p}\left(U_{\bullet}, K_{q}^{\mathrm{BM}}(-; \mathbb{Z} / n)\right)
$$

with the differential $d_{2}$ having bidegree $(2,1)$. Here, for any presheaf $F$ defined on the small étale site of $U$, the group $\check{\mathrm{H}}_{\text {ét }}^{p}\left(U_{\bullet}, F\right)$ denotes the $p$-th cohomology group of the complex

$$
F\left(U_{0}\right) \rightarrow F\left(U_{1}\right) \rightarrow \cdots,
$$

where the maps are given by the alternating sum of coboundary maps in the usual manner. Ideally, Theorem 7.8 would imply that this spectral sequence converges to $K_{q-p}^{\mathrm{BM}}(U ; \mathbb{Z} / n)$; however, the convergence of the spectral sequence is uncertain in general (cf. [6, IX.5]).

\section{DuAlity For SMOOTh VARIETIES}

In this section we define a map

$$
\gamma_{Y}: \bigoplus_{p} K_{p}^{\mathrm{BM}}(Y ; \mathbb{Z} / n) \rightarrow \bigoplus_{p} K_{\text {geom }}^{-p}(Y ; \mathbb{Z} / n)
$$

of graded abelian groups, for any smooth, quasi-projective $k$-variety $Y$, which is natural with respect to quasi-finite morphisms. (The functor $K_{*}^{\mathrm{BM}}(-; \mathbb{Z} / n$ ) is shown to be contravariantly functorial with respect to such morphisms below.) Here, we assume $k$ is algebraically closed and $n \geq 1$ is relatively prime to the exponential characteristic of $k$. (One can extend the definition of $\gamma_{Y}$ to theories with integral coefficients and to non-algebraically closed fields $k$, provided one assumes $k$ admits resolutions of singularities.) Moreover, we prove $\gamma_{Y}$ is a map of $K_{\text {geom }}^{*}(Y)$-modules and establish the key formula

$$
\gamma_{Y}(a) \cap b=\gamma_{Y}(b) \cap a
$$

for all $a, b \in K_{*}^{\mathrm{BM}}(Y ; \mathbb{Z} / n)$. We then define the notion of a fundamental class for $\mathbb{Z} / n$ coefficients of such a variety $Y$. By definition this is an element $[Y]$ of $K_{2 d}^{\mathrm{BM}}(Y ; \mathbb{Z} / n)$ such that $\gamma_{Y}([Y]) \in K_{\text {geom }}^{2 d}(Y ; \mathbb{Z} / n)$ is equal to the $d$-th power $\beta^{d}$ of 
the so-called Bott element $\beta$, where $d=\operatorname{dim}(Y)$. Taking the cap product with a fundamental class $[Y]$ defines a map

$$
K_{\text {geom }}^{p}(Y ; \mathbb{Z} / n) \stackrel{-\cap[Y]}{\longrightarrow} K_{2 d-p}^{\mathrm{BM}}(Y ; \mathbb{Z} / n)
$$

which, as we shall prove, is an inverse of $\gamma_{Y}$ up to multiplication by $\beta^{d}$. This then gives a form of "duality" relating $K$-homology and $K$-theory with finite coefficients for varieties with fundamental classes. In the next section, we prove every smooth, quasi-projective variety defined over an algebraically closed field has a fundamental class in this sense. Taken together with the results proved in this section, the existence of fundamental classes leads directly to the main results of this paper.

In order to define the map $\gamma_{Y}$, we need to introduce models for the $K$-theory spectra under consideration that use perfect complexes and the results and constructions of Waldhausen 31 and Thomason-Trobaugh 28.

Definition 8.1. Let $Y$ and $X$ be quasi-projective $k$-varieties and $U \longmapsto Y$ a locally closed subscheme. We define $\mathcal{P}^{q f W}(X,(U, Y))$ to be the category consisting of perfect complexes (cf. [28, 2.2.10]) of coherent $\mathcal{O}_{X \times U}$-modules $M_{\bullet}$ on $X \times U$ such that each $M_{n}$ is $\mathcal{O}_{X}$-flat and which satisfy the condition the that closure of the support of $M_{\bullet}$ in $X \times Y$ is quasi-finite over $X$. (Recall that the support of a complex $M_{\bullet}$ is defined by those points $z \in X \times U$ such that the localization of $M_{\bullet}$ at $z$ is not exact.) We endow $\mathcal{P}^{q f W}(X,(U, Y))$ with the structure of a "complicial biWaldhausen category" [28, 1.2.11] by viewing it as a subcategory of the category of all perfect complexes $M_{\bullet}$ on $X \times U$ [28, 3.5]. We write $\mathcal{K}^{q f W}(X,(U, Y))$ for the $K$-theory spectrum associated to $\mathcal{P}^{q f W}(X,(U, Y))$ as defined by Waldhausen 31] (see also [28, 1.5.2]).

If $U=Y$, we write $\mathcal{P}^{q f W}(X, U)$ and $\mathcal{K}^{q f W}(X, U)$ instead of $\mathcal{P}^{q f W}(X,(U, U))$ and $\mathcal{K}^{q f W}(X,(U, U))$.

We define $\mathcal{P}^{q f W f l}(X,(U, Y))$ to be the subcategory of $\mathcal{P}^{q f W}(X,(U, Y))$ consisting of complexes $M^{\bullet}$ satisfying the additional property that each $M^{n}$ is flat as an $\mathcal{O}_{U \times X}$-module; we write $\mathcal{K}^{q f W f l}(X,(U, Y))$ for the corresponding $K$-theory spectrum. If $U=Y$, we write $\mathcal{P}^{q f W f l}(X, U)$ and $\mathcal{K}^{q f W f l}(X, U)$ for $\mathcal{P}^{q f W f l}(X,(U, Y))$ and $\mathcal{K}^{q f W f l}(X,(U, Y))$.

Observe that an object of $\mathcal{P}^{q f}(X, U)$ may be regarded as a perfect complex concentrated in degree zero provided $X$ and $U$ are smooth varieties. In other words, for such varieties we have a natural functor

$$
\mathcal{P}^{q f}(X, U) \rightarrow \mathcal{P}^{q f W}(X, U)
$$

which determines a natural map of spectra

$$
\mathcal{K}^{q f}(X, U) \rightarrow \mathcal{K}^{q f W}(X, U)
$$

Also, the evident inclusion of categories induces a natural map of spectra

$$
\mathcal{K}^{q f W f l}(X, U) \rightarrow \mathcal{K}^{q f W}(X, U) .
$$

Proposition 8.2. Let $U$ be a smooth, quasi-projective variety defined over an algebraically closed field $k$ and assume $n \geq 1$ is an integer relatively prime to the exponential characteristic of $k$. Then the maps of simplicial spectra

$$
\left(d \mapsto \mathcal{K}^{q f}\left(\Delta^{d}, U\right)\right) \longrightarrow\left(d \mapsto \mathcal{K}^{q f W}\left(\Delta^{d}, U\right)\right) \longleftarrow\left(d \mapsto \mathcal{K}^{q f W f l}\left(\Delta^{d}, U\right)\right)
$$


induce isomorphisms upon taking homotopy groups with $\mathbb{Z} / n$ coefficients. Moreover, these isomorphisms are contravariantly natural in $U$ for quasi-finite, flat morphisms.

Proof. The right-hand arrow is shown to be a degree-wise weak equivalence as in the proof of [28, 3.5], and thus it induces an isomorphism with homotopy groups in arbitrary coefficients.

Choose a projective closure $U \longmapsto \bar{U}$ and consider the commutative diagram of simplicial spectra

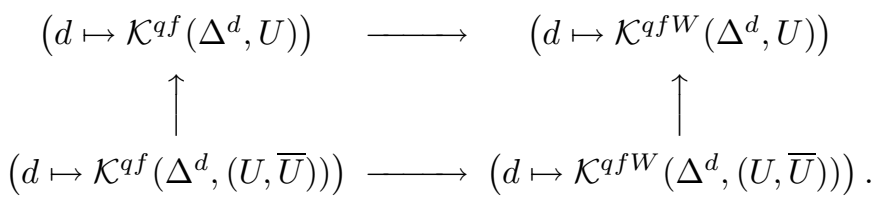

The left-hand vertical arrow induces an isomorphism upon taking $\pi_{p}(-; \mathbb{Z} / n)$, for all $p \geq 0$, by part (1) of Corollary 5.3. A nearly identical proof shows the right-hand vertical arrow also induces an isomorphism upon applying $\pi_{p}(-; \mathbb{Z} / n)$. It therefore suffices to prove the bottom horizontal arrow is a weak equivalence.

For each fixed $d$, we have a commutative diagram

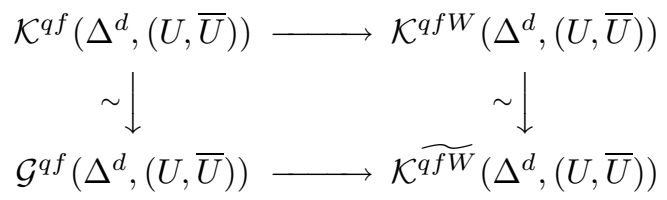

of spectra. Here, $\widetilde{\mathcal{K}^{q f W}}\left(\Delta^{d},(U, \bar{U})\right)$ denotes the $K$-theory of the complicial biWaldhausen category consisting of all perfect complexes of coherent $\mathcal{O}_{\Delta^{d} \times U^{-}}$-modules $F^{\bullet}$ such that the closure of the support of $F^{\bullet}$ in $\Delta^{d} \times \bar{U}$ is quasi-finite over $X$. (In other words, we drop the hypothesis that each $F^{n}$ be $\mathcal{O}_{\Delta^{d}}$-flat.) Objects of $\mathcal{M}^{q f}\left(\Delta^{d},(U, \bar{U})\right)$ are viewed as perfect complexes concentrated in degree 0 , which justifies the existence of the bottom arrow of this diagram. The left-hand vertical arrow of this diagram is a weak equivalence by Proposition 5.4 and the right-hand vertical arrow is shown to be a weak equivalence as in the proof of [28, 3.5]. Finally, we claim that each of the spectra on the bottom row is weakly equivalent to $\lim _{Z} \mathcal{G}(Z)$, where $Z$ ranges over closed subschemes of $\Delta^{d} \times U$ whose closures in $\Delta^{d} \times \bar{U}$ are quasi-finite over $\Delta^{d}$, and thus the bottom map is a weak equivalence. This is evident for $\mathcal{G}^{q f}\left(\Delta^{d},(U, \bar{U})\right)$, and for $\mathcal{K}^{\widetilde{q f W}}\left(\Delta^{d},(U, \bar{U})\right)$ the assertion follows from the Thomason Localization Theorem [28, 5.1].

The fact that the maps are natural for quasi-finite, flat morphisms is evident.

Definition 8.3. Given a quasi-projective $k$-variety $U$, we write $\mathcal{P}^{W}(U)$ (respectively, $\mathcal{P}^{W f l}(U)$ ) for the complicial biWaldhausen category of all perfect complexes of coherent sheaves $M^{\bullet}$ on $U$ (respectively, all perfect complexes of coherent sheaves $M^{\bullet}$ such that each $M^{n}$ is $\mathcal{O}_{U}$-flat). (Thus, $\mathcal{P}^{W f l}(U)=\mathcal{P}^{q f W}(U$, Spec $k)$.) We write $\mathcal{K}^{W}(U)$ and $\mathcal{K}^{W f l}(U)$ for the associated $K$-theory spectra.

Note that there is a natural inclusion $\mathcal{P}(U) \longmapsto \mathcal{P}^{W f l}(U)$ (given by regarding a vector bundle as a chain complex concentrated in degree zero) and hence a natural map $\mathcal{K}(U) \rightarrow \mathcal{K}^{W f l}(U)$ of associated spectra. 
Lemma 8.4. For any quasi-projective variety $U$, the inclusion $\mathcal{P}(U) \longmapsto \mathcal{P}^{W f l}(U)$ induces a natural weak equivalence

$$
\mathcal{K}_{\text {geom }}(U) \stackrel{\sim}{\longrightarrow}\left|d \mapsto \mathcal{K}^{W f l}\left(\Delta^{d} \times U\right)\right| .
$$

Proof. The underlying map of simplicial spectra is a degree-wise weak equivalence by [28, 3.5 and 3.10$]$.

The inclusion $\mathcal{P}^{q f W f l}(X, U) \longmapsto \mathcal{P}^{W f l}(X \times U)$ is a map of complicial biWaldhausen categories, and thus it induces a natural map of $K$-theory spectra. The map we seek, $\gamma_{U}$, is defined in terms of this map of spectra by using the weak equivalences established above.

Definition 8.5. Let $U$ be a smooth, quasi-projective variety defined over an algebraically closed field $k$ and assume $n \geq 1$ is an integer relatively prime to the exponential characteristic of $k$. Define

$$
\gamma_{U}: K_{q}^{\mathrm{BM}}(U ; \mathbb{Z} / n) \rightarrow K_{\text {geom }}^{-q}(U ; \mathbb{Z} / n),
$$

for any $q$, via the chain of maps

$$
\begin{aligned}
& K_{q}^{\mathrm{BM}}(U ; \mathbb{Z} / n) \stackrel{\cong}{\longrightarrow} \pi_{q}\left(\left|d \mapsto \mathcal{K}^{q f W}\left(\Delta^{d}, U\right)\right| ; \mathbb{Z} / n\right) \\
& \pi_{q}\left(\left|d \mapsto \mathcal{K}^{q f W f l}\left(\Delta^{d}, U\right)\right| ; \mathbb{Z} / n\right) \\
& \downarrow \\
& K_{\text {geom }}^{-q}(U ; \mathbb{Z} / n) \stackrel{\downarrow}{\cong} \pi_{q}\left(\left|d \mapsto \mathcal{K}^{W f l}\left(\Delta^{d} \times U\right)\right| ; \mathbb{Z} / n\right),
\end{aligned}
$$

where the displayed isomorphisms are established in Proposition 8.2 and Lemma 8.4.

Remark 8.6. In the case where $Y$ is smooth and projective and $n$ satisfies (Condition 2-3), the map $\gamma_{Y}$ of Definition 8.5 admits a simpler description in terms of standard cohomological operations. Namely, for such a $Y$, let

$$
\left[\delta_{Y}\right] \in K_{\text {geom }}^{0}(Y \times Y) \cong K_{0}^{\mathrm{alg}}(Y)
$$

be the class of the structure sheaf of the diagonal closed subscheme $Y \longmapsto Y \times Y$, and recall that the slant product is the pairing

$$
-/-: K_{\text {geom }}^{p}(X \times Y ; \mathbb{Z} / n) \otimes K_{q}^{\mathrm{hom}}(Y ; \mathbb{Z} / n) \rightarrow K_{\text {geom }}^{p-q}(X ; \mathbb{Z} / n)
$$

defined by the formula $x / y=p_{*}\left(x \bullet q^{*} y\right)$, where $p: X \times Y \rightarrow Y$ is the canonical projection map and $q: X \rightarrow \operatorname{Spec} k$ is the structure map (cf. [10, 2.4]). Then we have

$$
\gamma_{Y}=\left[\delta_{Y}\right] /-: K_{q}^{\mathrm{hom}}(Y ; \mathbb{Z} / n) \rightarrow K_{\text {geom }}^{-q}(Y ; \mathbb{Z} / n) .
$$

In addition to enabling the definition $\gamma_{U}$, the isomorphisms of Proposition 8.2 also allow us to define Gysin maps in a more general setting than before and, consequently, allows for the construction of a product rule for $K^{\mathrm{BM}}(-; \mathbb{Z} / n)$. Concerning the Gysin maps, given a quasi-finite (but possibly not flat) map $f: V \rightarrow U$ of smooth, quasi-projective $k$-varieties, pullback along $i d_{X} \times f$ determines a natural exact functor

$$
f^{*}: \mathcal{P}^{q f W f l}(X, U) \rightarrow \mathcal{P}^{q f W f l}(X, V),
$$


for any $X$, and hence a map of spectra

$$
f^{*}: \mathcal{K}^{q f W f l}(X, U) \rightarrow \mathcal{K}^{q f W f l}(X, V) .
$$

Replacing $X$ with $\Delta^{\bullet}$ and using the isomorphisms of Proposition 8.2, this natural map of spectra determines a map

$$
f^{*}: K_{q}^{\mathrm{BM}}(U ; \mathbb{Z} / n) \rightarrow K_{q}^{\mathrm{BM}}(V ; \mathbb{Z} / n),
$$

for all $q$, provided $k$ is algebraically closed and $n \geq 1$ is relatively prime to the exponential characteristic of $k$. If $f$ happens to be flat, the Gysin map defined here coincides with the pullback map defined in Proposition 3.8.

Proposition 8.7. If $f: V \rightarrow U$ is a quasi-finite morphism of smooth, quasiprojective $k$-varieties, then the diagram

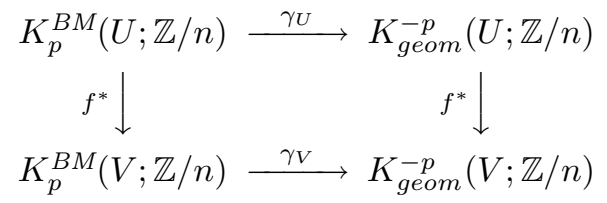

commutes. Similarly, if $i: Z \longmapsto X$ is a regular closed immersion of smooth, quasiprojective $k$-varieties, then the diagram

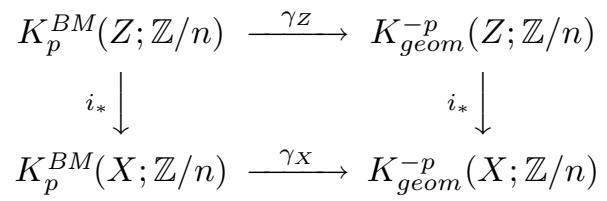

commutes, where the map $i_{*}$ on the right is the usual pushforward map in $K$-theory for a regular closed immersion.

Proof. Using the weak equivalences of Proposition 8.2 and Lemma 8.4, the commutativity of the first diagram follows from the the fact that the diagram

$$
\begin{array}{cc}
\mathcal{K}^{q f W f l}(Y, U) & \longrightarrow \mathcal{K}^{W f l}(Y \times U) \\
\left(i d_{Y} \times f\right)^{*} \downarrow & \left(i d_{Y} \times f\right)^{*} \downarrow \\
\mathcal{K}^{q f W f l}(Y, V) & \longrightarrow \mathcal{K}^{W f l}(Y \times V)
\end{array}
$$

commutes for all smooth $Y$ and is natural in $Y$. Similarly, the commutativity and naturality of

$$
\begin{aligned}
\mathcal{K}^{q f W}(Y, Z) & \longrightarrow \mathcal{K}^{W}(Y \times Z) \\
\left(i d_{Y} \times i\right)_{*} \downarrow & \left(i d_{Y} \times i\right)_{*} \downarrow \\
\mathcal{K}^{q f W}(Y, X) & \longrightarrow \mathcal{K}^{W}(Y \times X)
\end{aligned}
$$

for all smooth $Y$ implies the commutativity of the second diagram. (The fact that $i$ is a regular closed immersion implies that the pushforward along $i$ of a perfect complex on $Y \times Z$ is a perfect complex on $Y \times X$.)

Proposition 8.8. Let $U$ be a smooth, quasi-projective variety defined over an algebraically closed field $k$ and assume $n \geq 1$ is an integer relatively prime to the exponential characteristic of $k$. Then the map

$$
\gamma_{U}: K_{m}^{B M}(U ; \mathbb{Z} / n) \rightarrow K_{\text {geom }}^{-m}(U ; \mathbb{Z} / n)
$$


is a homomorphism of $K_{\text {geom }}^{*}(U)$-modules. If additionally $n$ satisfies (Condition $2-3)$, it is a map of $K_{\text {geom }}^{*}(U ; \mathbb{Z} / n)$-modules.

Proof. Using the equivalences of Proposition 8.2 and Lemma 8.4 the first assertion follows from the commutativity of the diagram of spectra

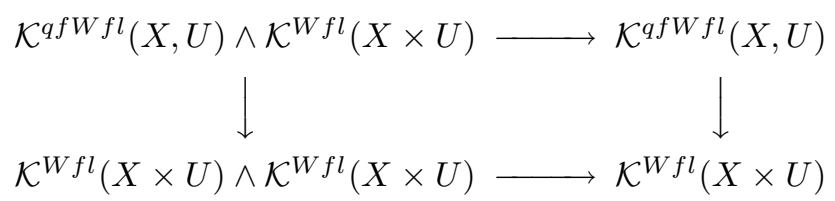

whose horizontal maps are induced by a tensor product of perfect complexes and whose vertical maps are induced by the evident inclusions of categories. The final assertion holds since the mod- $n$ Moore space admits an appropriate coproduct rule for such values of $n$.

The isomorphisms of Proposition 8.2 also allows us to define certain pairings for $K^{\mathrm{BM}}$. Namely, given smooth varieties $U$ and $V$, there is a natural biexact pairing of categories

$$
\mathcal{P}^{q f W f l}(X, U) \times \mathcal{P}^{q f W f l}(X, V) \rightarrow \mathcal{P}^{q f W f l}(X, U \times V),
$$

given by taking external tensor product of complexes. That is, if $M^{\bullet}\left(\right.$ resp., $\left.N^{\bullet}\right)$ is a degree-wise flat perfect complex on $X \times U$ (resp., $X \times V$ ), then $p^{*} M^{\bullet} \otimes_{\mathcal{O}_{X \times U \times V}}$ $q^{*} N^{\bullet}$ is a degree-wise flat perfect complex on $X \times U \times V$. (Here, $p$ and $q$ are the evident projections.) Moreover, the quasi-finiteness of support is preserved under this operation, and so we have a biexact pairing as indicated above. We thus obtain a natural (in $X$ ) pairing of spectra

$$
\mathcal{K}^{q f W f l}(X, U) \wedge \mathcal{K}^{q f W f l}(X, V) \rightarrow \mathcal{K}^{q f W f l}(X, U \times V) .
$$

Replacing $X$ with $\Delta^{\bullet}$, taking homotopy groups with coefficients in $\mathbb{Z} / n$ (with $n \not \equiv 2$ $(\bmod 4)$ ), and using the isomorphisms of Proposition 8.2, we obtain the pairing

$$
\mu_{U, V}: K_{p}^{\mathrm{BM}}(U ; \mathbb{Z} / n) \otimes K_{p}^{\mathrm{BM}}(V ; \mathbb{Z} / n) \rightarrow K_{p}^{\mathrm{BM}}(U \times V ; \mathbb{Z} / n) .
$$

This pairing is commutative and associative in the evident sense provided $n$ satisfies (Condition 2-3).

The next result will be used in the next section to establish the existence of fundamental classes for affine space (cf. Lemma 9.1).

Proposition 8.9. Let $U$ and $V$ be smooth, quasi-projective varieties defined over an algebraically closed field. Assume $n \geq 1$ is an integer relative prime to the exponential characteristic of $k$ and that $n$ satisfies (Condition 2-3). Then the pairing

$$
\mu_{U, V}: K_{i}^{B M}(U ; \mathbb{Z} / n) \otimes K_{j}^{B M}(V ; \mathbb{Z} / n) \rightarrow K_{i+j}^{B M}(U \times V ; \mathbb{Z} / n)
$$

defined above is natural $U$ and $V$ for quasi-finite morphisms, and it satisfies the equation

$$
\pi_{1}^{*} \gamma_{U}(a) \cup \pi_{2}^{*} \gamma_{V}(b)=\gamma_{U \times V}\left(\mu_{U, V}(a \otimes b)\right) .
$$

Proof. Naturality holds since the pairing

$$
\mathcal{K}^{q f W f l}(X, U) \wedge \mathcal{K}^{q f W f l}(X, V) \rightarrow \mathcal{K}^{q f W f l}(X, U \times V)
$$


is natural in $U$ and $V$ with respect to quasi-finite pullback, for any $X$. To establish the identity, note there is a commutative diagram of spectra

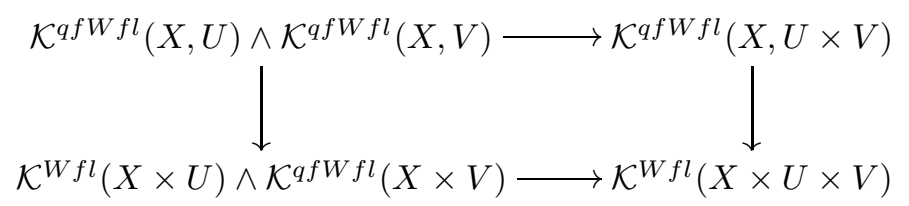

in which the horizontal maps are given by taking the tensor product of perfect complexes and the vertical maps are induced by the evident inclusions of categories. Replacing $X$ with $\Delta^{\bullet}$ and taking homotopy groups with $\mathbb{Z} / n$ coefficients, it is clear that under the equivalences of Proposition 8.2 and Lemma 8.4 that the composition of the lower-left vertical arrow with the bottom horizontal arrow in this diagram induces the map

$$
K_{p}^{\mathrm{BM}}(U ; \mathbb{Z} / n) \otimes K_{q}^{\mathrm{BM}}(V ; \mathbb{Z} / n) \rightarrow K_{\text {geom }}^{-p-q}(U \times V ; \mathbb{Z} / n)
$$

given by $a \otimes b \mapsto \pi_{1}^{*}\left(\gamma_{U}(a)\right) \cup \pi_{2}^{*}\left(\gamma_{V}(b)\right)$. The other composition in this square induces the map $a \otimes b \mapsto \gamma_{U \times V}\left(\mu_{U, V}(a \otimes b)\right)$.

Composing the pairing $\mu_{U, U}$ with pullback along the diagonal embedding $\Delta$ : $U \longmapsto U \times U$, we obtain the pairing

$$
\mu_{U}: K_{p}^{\mathrm{BM}}(U ; \mathbb{Z} / n) \otimes K_{q}^{\mathrm{BM}}(U ; \mathbb{Z} / n) \rightarrow K_{p+q}^{\mathrm{BM}}(U ; \mathbb{Z} / n) .
$$

Under the isomorphisms of Proposition 8.2 the pairing $\mu_{U}$ may equivalently be defined as the map induced by the natural biexact pairing

$$
\mathcal{P}^{q f W f l}(X, U) \times \mathcal{P}^{q f W f l}(X, U) \rightarrow \mathcal{P}^{q f W f l}(X, U)
$$

sending a pair of complexes $\left(M^{\bullet}, N^{\bullet}\right)$ to $M^{\bullet} \otimes_{\mathcal{O}_{X \times U}} N^{\bullet}$.

Proposition 8.10. Let $U$ be a smooth, quasi-projective variety defined over an algebraically closed field $k$. Assume $n \geq 1$ is an integer relatively prime to the exponential characteristic of $k$ and that $n$ satisfies (Condition 2-3). Then the pairing

$$
\mu_{U}: K_{p}^{B M}(U ; \mathbb{Z} / n) \otimes K_{q}^{B M}(U ; \mathbb{Z} / n) \rightarrow K_{p+q}^{B M}(U ; \mathbb{Z} / n)
$$

defined above satisfies the equation

$$
a \cap \gamma_{U}(b)=\mu_{U}(a \otimes b)=(-1)^{p q} b \cap \gamma_{U}(a) .
$$

Proof. Observe that there is a commutative diagram of spectra

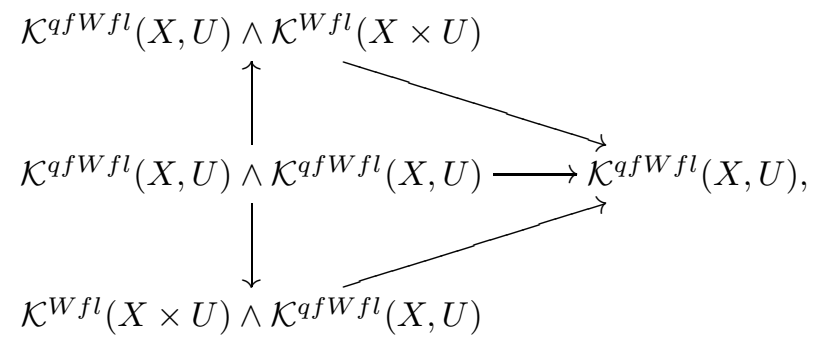

where the two diagonal arrows and the horizontal arrow are given by tensor product of perfect complexes and the vertical arrows are induced by the evident inclusion of categories. Under the isomorphisms given by Proposition 8.2 and Lemma 8.4, the 
composition of the lower vertical map and the lower diagonal map in this diagram induces the pairing

$$
K_{p}^{\mathrm{BM}}(U ; \mathbb{Z} / n) \otimes K_{q}^{\mathrm{BM}}(U ; \mathbb{Z} / n) \rightarrow K_{p+q}^{\mathrm{BM}}(U ; \mathbb{Z} / n)
$$

which sends $a \otimes b$ to $\gamma_{U}(a) \cap b$. Similarly, the composition of the upper vertical map and upper diagonal map induces the pairing $a \otimes b \mapsto(-1)^{p q} \gamma_{U}(b) \cap a$, and the horizontal map induces $a \otimes b \mapsto \mu_{U}(a \otimes b)$.

We now define the notion of a fundamental class for a smooth, quasi-projective variety. The mere existence of such a class in conjunction with the preceding results immediately gives the desired "duality" result discussed above. In the next section, we establish the existence of such classes for all smooth, quasi-projective varieties.

Definition 8.11. Let $k$ be an algebraically closed field, and $n \geq 1$ any integer relatively prime to the exponential characteristic of $k$. Choose $b \in \mu_{n}(k)$ to be a primitive $n$-th root of unity. Define the Bott element $\beta \in K_{\text {geom }}^{2}(k ; \mathbb{Z} / n)$ to be the unique element mapping to $b$ under the boundary map

$$
K_{\text {geom }}^{2}(k ; \mathbb{Z} / n) \rightarrow K_{\text {geom }}^{1}(k) \cong k^{\times} .
$$

We also write $\beta$ for the image of $\beta$ under the natural map $K_{\text {geom }}^{2}(k ; \mathbb{Z} / n) \rightarrow$ $K_{\text {geom }}^{2}(Y ; \mathbb{Z} / n)$ for any $k$-scheme $Y$.

Definition 8.12. Let $k$ be an algebraically closed field, $Y$ a smooth, quasi-projective $k$-variety of pure dimension $d$, and $n \geq 1$ an integer relatively prime to the exponential characteristic of $k$. A fundamental class of $Y$ for $\mathbb{Z} / n$ coefficients is an element $[Y] \in K_{2 d}^{\mathrm{BM}}(Y ; \mathbb{Z} / n)$ such that $\gamma_{Y}([Y])=\beta^{d}$.

Theorem 8.13. Let $k$ be an algebraically closed field, $Y$ a smooth, quasi-projective $k$-variety of pure dimension $d$, and assume $n \geq 1$ is an integer relatively prime to the exponential characteristic of $k$ and that $n$ satisfies (Condition 2-3). If $Y$ has a fundamental class $[Y] \in K_{2 d}^{B M}(Y ; \mathbb{Z} / n)$, then the composition of

$$
K_{i}^{B M}(Y ; \mathbb{Z} / n) \stackrel{\gamma_{Y}}{\longrightarrow} K_{\text {geom }}^{-i}(Y ; \mathbb{Z} / n) \stackrel{-\cap[Y]}{\longrightarrow} K_{2 d+i}^{B M}(Y ; \mathbb{Z} / n)
$$

coincides with $\beta^{d} \cap-$ and the composition of

$$
K_{\text {geom }}^{j}(Y ; \mathbb{Z} / n) \stackrel{-\cap[Y]}{\longrightarrow} K_{2 d-j}^{B M}(Y ; \mathbb{Z} / n) \stackrel{\gamma_{Y}}{\longrightarrow} K_{\text {geom }}^{j-2 d}(Y ; \mathbb{Z} / n)
$$

coincides with $\beta^{d} \cup-$. Consequently, as a $K_{\text {geom }}^{*}(Y ; \mathbb{Z} / n)[1 / \beta]$-module, the module $K_{*}^{B M}(Y ; \mathbb{Z} / n)[1 / \beta]$ is graded-free of rank one with basis given by the fundamental class.

Proof. For $x \in K_{i}^{\mathrm{BM}}(Y ; \mathbb{Z} / n)$ we have $\gamma_{Y}(x) \cap[Y]=\gamma_{Y}([Y]) \cap x=\beta^{d} \cap x$ by Proposition 8.10 (using that $[Y]$ has even degree). Similarly for $x \in K_{\text {geom }}^{j}(Y ; \mathbb{Z} / n)$, we have $\gamma_{Y}(x \cap[Y])=x \cup \gamma_{Y}([Y])=\beta^{d} \cup x$ by Proposition 8.8 (using that $\beta$ has even degree). The final assertion clearly follows.

\section{Fundamental Classes For Smooth VARIEties}

In this section we establish that every smooth, quasi-projective variety $Y$ over an algebraically closed field $k$ has a fundamental class for $\mathbb{Z} / n$ coefficients, where $n \geq 1$ is relatively prime to the exponential characteristic of $k$. 
Lemma 9.1. Let $k$ be an algebraically closed field and assume $n \geq 1$ is an integer relatively prime to the exponential characteristic of $k$ satisfying (Condition 2-3). Then for all $m \geq 0$ the variety $\mathbb{A}^{m}$ has a fundamental class $\left[\mathbb{A}^{m}\right]$ for $\mathbb{Z} / n$ coefficients. In fact, $K_{*}^{B M}\left(\mathbb{A}^{m} ; \mathbb{Z} / n\right)$ is a graded-free $K_{\text {geom }}^{-*}(\operatorname{Spec} k ; \mathbb{Z} / n)$-module of rank one generated by $\left[\mathbb{A}^{m}\right]$.

Proof. For $m=0$, the assertion is obvious, since $\mathcal{K}^{B M}(\operatorname{Spec} k)=\mathcal{K}_{\text {geom }}(\operatorname{Spec} k)$. Suppose there was a fundamental class $\left[\mathbb{A}^{1}\right]$ for $\mathbb{A}^{1}$ belonging to $K_{2}^{\mathrm{BM}}\left(\mathbb{A}^{1} ; \mathbb{Z} / n\right)$. Then by applying Proposition 8.9 repeatedly, we would have that the element of $K_{2 m}^{\mathrm{BM}}\left(\mathbb{A}^{m} ; \mathbb{Z} / n\right)$ obtained by multiplying $\left[\mathbb{A}^{1}\right]$ with itself $m$ times using the pairing $\mu$ defines a fundamental class for $\mathbb{A}^{m}$. (Note that we have not proven $\mu$ is associative — but any manner of multiplying $\left[\mathbb{A}^{1}\right]$ with itself suffices.) Thus, to prove $\mathbb{A}^{m}$ has a fundamental class for all $m \geq 0$, it suffices to prove $\mathbb{A}^{1}$ has a fundamental class.

For any finitely generated smooth $k$-algebra $R$, we claim there is a homotopy fibration sequence of the form

$$
\mathcal{K}^{q f}\left(\operatorname{Spec} R, \mathbb{A}^{1}\right) \rightarrow \mathcal{K}(R[x]) \rightarrow \mathcal{K}\left(S^{-1} R[x]\right),
$$

where $S$ is the multiplicatively closed subset of $R[x]$ consisting of polynomials of unit content (i.e., those $r_{n} x^{n}+\cdots+r_{1} x+r_{0}$ such that $r_{0}, \ldots, r_{n}$ generate $R$ as an ideal). To see this, one argues as in the proof of Proposition 4.11 above. Namely, we have a fibration sequence of the form

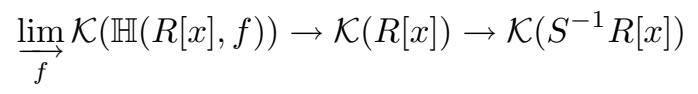

by [11, Theorem on p. 229], where $f$ ranges over elements of $S$ (ordered by division). A nearly identical argument to that given in Proposition 4.11 shows that the category $\lim _{f} \mathbb{H}(R[x], f)$ (which is a full subcategory of $\mathcal{M}(R[x])$ ) coincides with $\mathcal{P}^{q f}\left(\operatorname{Spec} R, \mathbb{A}^{1}\right)$. Replacing $R$ by the simplicial ring $d \mapsto k\left[\Delta^{d}\right]$, where $k\left[\Delta^{d}\right]=k\left[y_{0}, \ldots, y_{d}\right] /\left\langle\left(\sum_{i} y_{i}\right)-1\right\rangle$, and taking geometric realizations gives a fibration sequence of the form

$$
\left|d \mapsto \mathcal{K}^{q f}\left(\Delta^{d}, \mathbb{A}^{1}\right)\right| \rightarrow\left|d \mapsto \mathcal{K}\left(\Delta^{d} \times \mathbb{A}^{1}\right)\right| \rightarrow\left|d \mapsto \mathcal{K}^{q f}\left(S_{d}^{-1}\left(k\left[\Delta^{d}\right]\right)[x]\right)\right|,
$$

where $S_{d}$ is the set of polynomials in $k\left[\Delta^{d}\right][x]$ of unit content. (Observe that the map $K_{0}(R[x]) \rightarrow K_{0}\left(S^{-1} R[x]\right)$ is surjective since $R[x]$ is regular.) Since the simplicial ring $d \mapsto S_{d}^{-1} k \Delta^{d}[x]$ satisfies the primitive criterion degree-wise, the space

$$
\left|d \mapsto \mathcal{K}^{q f}\left(S_{d}^{-1} k \Delta^{d}[x]\right)\right|
$$

is homotopy equivalent to the discrete space $\mathbb{Z}$ by [35, 2.4]. It follows that the map

$$
\left|d \mapsto \mathcal{K}^{q f}\left(\Delta^{d}, \mathbb{A}^{1}\right)\right| \rightarrow\left|d \mapsto \mathcal{K}\left(\Delta^{d} \times \mathbb{A}^{1}\right)\right|
$$

induces an isomorphism on $\pi_{n}(-; A)$ for any $n \geq 1$ and any group of coefficients $A$. This map defines the map $\gamma_{\mathbb{A}^{1}}$ introduced in the previous section, and it follows that $\mathbb{A}^{1}$ has a fundamental class.

To prove the final assertion, observe that the spectral sequence of Theorem 7.6 for affine space degenerates at the $E_{2}$ terms to give

$$
K_{i}^{\mathrm{BM}}\left(\mathbb{A}^{m} ; \mathbb{Z} / n\right) \cong \mathrm{H}_{\text {ét }}^{0}\left(\mathbb{A}^{m} ; \mu_{n}^{\otimes i / 2}(k)\right) \cong \mu_{n}^{\otimes i / 2}(k), \quad \text { for } i \geq 2 m,
$$


and $K_{i}^{\mathrm{BM}}\left(\mathbb{A}^{m} ; \mathbb{Z} / n\right)=0$ for $i<2 m$. (As usual, one interprets $\mu_{n}^{\otimes i / 2}$ as being 0 for $i$ odd.) By Proposition 8.8, the map

$$
\gamma_{\mathbb{A}^{m}}: K_{*}^{\mathrm{BM}}\left(\mathbb{A}^{m} ; \mathbb{Z} / n\right) \rightarrow K_{\text {geom }}^{-*}\left(\mathbb{A}^{m} ; \mathbb{Z} / n\right) \cong K_{\text {geom }}^{-*}(\operatorname{Spec} k ; \mathbb{Z} / n) \cong \mathbb{Z} / n[\beta]
$$

is a graded $\mathbb{Z} / n[\beta]$-module homomorphism, and by Theorem 8.13 , the composition of

$$
K_{\text {geom }}^{-*}\left(\mathbb{A}^{m} ; \mathbb{Z} / n\right) \stackrel{-\cap\left[\mathbb{A}^{m}\right]}{\longrightarrow} K_{*}^{\mathrm{BM}}\left(\mathbb{A}^{m} ; \mathbb{Z} / n\right) \stackrel{\gamma_{\mathbb{A}} m}{\longrightarrow} K_{\text {geom }}^{2 m-*}\left(\mathbb{A}^{m} ; \mathbb{Z} / n\right)
$$

is multiplication by $\beta^{d}$. In particular, for $i \geq 2 m$ and even, the map

$$
\gamma_{\mathbb{A}^{m}}: K_{i}^{\mathrm{BM}}\left(\mathbb{A}^{m} ; \mathbb{Z} / n\right) \rightarrow K_{\text {geom }}^{-i}\left(\mathbb{A}^{m} ; \mathbb{Z} / n\right)
$$

is a surjection of groups isomorphic to $\mathbb{Z} / n$, and hence must be an isomorphism. Since $K_{i}^{\mathrm{BM}}\left(\mathbb{A}^{m} ; \mathbb{Z} / n\right)=0$ for $i$ odd or $i<2 m$, it follows that $\gamma_{\mathbb{A}^{m}}$ is an isomorphism onto the ideal $\beta^{m} \cdot \mathbb{Z} / n[\beta]$. The desired result now follows from Theorem 8.13

Theorem 9.2. Let $k$ be an algebraically closed field and assume $n \geq 1$ is an integer relatively prime to the exponential characteristic of $k$ satisfying (Condition $2-3)$. Then for all $m \geq 0$, the map

$$
\gamma_{\mathbb{P}^{m}}: K_{p}^{B M}\left(\mathbb{P}^{m} ; \mathbb{Z} / n\right) \rightarrow K_{\text {geom }}^{-p}\left(\mathbb{P}^{m} ; \mathbb{Z} / n\right)
$$

is an isomorphism in degrees $p \geq 2 m$. In particular, the variety $\mathbb{P}^{m}$ has a fundamental class for $\mathbb{Z} / n$ coefficients.

Proof. We proceed by induction on $m$, with the case $m=0$ being obvious.

Let $j: \mathbb{A}^{m} \longmapsto \mathbb{P}^{m}$ be the canonical open embedding with closed complement $i: \mathbb{P}^{m-1} \longmapsto \mathbb{P}^{m}$. The spectral sequences of Theorem $\left[7.6\right.$ for $\mathbb{P}^{m}$ and $\mathbb{A}^{m}$ both degenerate at the $E_{2}$ terms and, as we have seen, the degeneration of the spectral sequence for $\mathbb{A}^{m}$ determines the isomorphism

$$
K_{2 m+2 i}^{\mathrm{BM}}\left(\mathbb{A}^{m} ; \mathbb{Z} / n\right) \cong \mathrm{H}_{\text {ét }}^{0}\left(\mathbb{A}^{m} ; \mu_{n}^{\otimes(m+i)}\right)=E_{2}^{m+i,-m-i},
$$

for all $i \geq 0$. The generation of the spectral sequence of $\mathbb{P}^{m}$ implies the existence of a filtration on $K_{2 m+2 i}^{\mathrm{BM}}\left(\mathbb{A}^{m} ; \mathbb{Z} / n\right)$, for $i \geq 0$, one of whose associated quotient groups is

$$
E_{2}^{m+i,-m-i}=\mathrm{H}_{\text {ét }}^{0}\left(\mathbb{P}^{m} ; \mu_{n}^{\otimes(m+i)}\right) .
$$

Since $j^{*}$ induces a map of spectral sequences and the map

$$
j^{*}: \mathrm{H}_{\text {êt }}^{0}\left(\mathbb{P}^{m} ; \mu_{n}^{\otimes(m+i)}\right) \rightarrow \mathrm{H}_{\text {ét }}^{0}\left(\mathbb{A}^{m} ; \mu_{n}^{\otimes(m+i)}\right)
$$

is an isomorphism, we have that

$$
j^{*}: K_{2 m+2 i}^{\mathrm{BM}}\left(\mathbb{P}^{m} ; \mathbb{Z} / n\right) \rightarrow K_{2 m+2 i}^{\mathrm{BM}}\left(\mathbb{A}^{m} ; \mathbb{Z} / n\right)
$$


is surjective for all $i \geq 0$. Since $K_{t}^{\mathrm{BM}}\left(\mathbb{A}^{m} ; \mathbb{Z} / n\right)=0$ for $t$ odd, the columns of the diagram

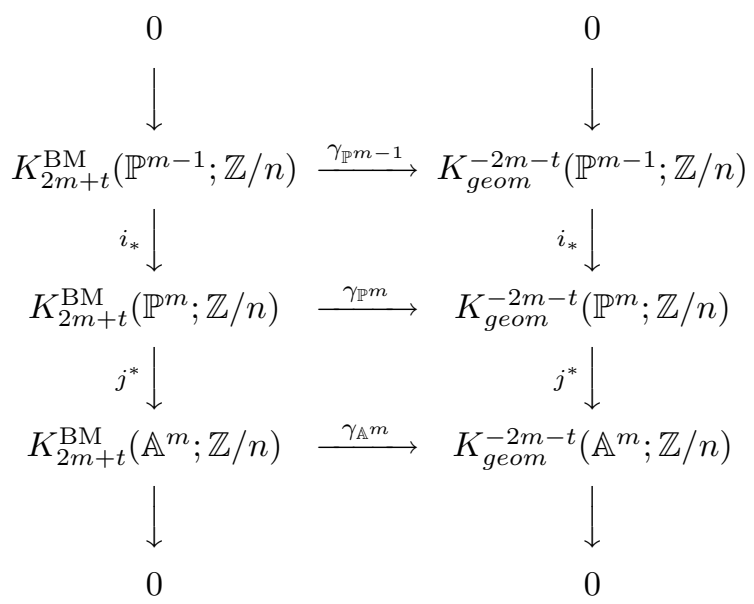

are exact. The diagram commutes by Proposition 8.7, and for all $t \geq 0$ the bottom horizontal map is an isomorphism by Lemma 9.1. Using induction and the Five Lemma, we have that

$$
\gamma_{\mathbb{P} m}: K_{2 m+t}^{\mathrm{BM}}\left(\mathbb{P}^{m} ; \mathbb{Z} / n\right) \rightarrow K_{\text {geom }}^{-2 m-t}\left(\mathbb{P}^{m} ; \mathbb{Z} / n\right)
$$

is an isomorphism for all $t \geq 0$.

In particular, taking $t=0$, the element $\beta^{m} \in K_{\text {geom }}^{-2 m}\left(\mathbb{P}^{m} ; \mathbb{Z} / n\right)$ lifts to a fundamental class $\left[\mathbb{P}^{m}\right] \in K_{2 m}^{\mathrm{BM}}\left(\mathbb{P}^{m} ; \mathbb{Z} / n\right)$.

Theorem 9.3. Let $k$ be an algebraically closed field and assume $n \geq 1$ is an integer relatively prime to the exponential characteristic of $k$ satisfying (Condition 2-3). Then every smooth, quasi-projective $k$-variety of pure dimension has a fundamental class for $\mathbb{Z} / n$ coefficients.

Proof. Let $U$ be any smooth, quasi-projective $k$-variety of pure dimension $d$. Let $U \subset Y$ be an open dense embedding into a projective variety $Y$ and choose a finite map $\pi: Y \rightarrow \mathbb{P}^{d}$. Then the restriction $f: U \rightarrow \mathbb{P}^{d}$ of $\pi$ to $U$ is quasi-finite (and hence flat by [18, 23.1]). It follows immediately from the naturality property of $\gamma_{U}$ established in Proposition 8.7 that $f^{*}\left(\left[\mathbb{P}^{d}\right]\right)$ is a fundamental class of $U$, where $\left[\mathbb{P}^{d}\right]$ is a fundamental class of $\mathbb{P}^{d}$ whose existence is guaranteed by Theorem 9.2,

Corollary 9.4. Let $k$ be an algebraically closed field and assume $n \geq 1$ is an integer relatively prime to the exponential characteristic of $k$ satisfying (Condition 2-3). For any smooth, quasi-projective $k$-variety $Y$, the natural map

$$
K_{*}^{B M}(Y ; \mathbb{Z} / n)[1 / \beta] \stackrel{\gamma_{Y}}{\longrightarrow} K_{\text {geom }}^{-*}(Y ; \mathbb{Z} / n)[1 / \beta]
$$

is an isomorphism.

Proof. This follows immediately from Theorems 8.13 and 9.3

\section{ThOMASON'S THEOREM FOR VARIETIES OVER ALGEBRAICALLY CLOSED FIELDS}

In this section we deduce Thomason's theorem for smooth varieties defined over an algebraically closed base field $k$ (with the usual conditions on the coefficients $\mathbb{Z} / n)$. Namely, for such a variety $U$, we prove the existence of a spectral sequence 
with $E_{2}$ terms given by the appropriate étale cohomology groups converging to $K_{*}(U ; \mathbb{Z} / n)[1 / \beta]$. The proof of this theorem is essentially given by combining Theorems 7.6 [8.13, and 9.3 Indeed, in order to establish the existence of the above-mentioned spectral sequence, the only additional component we require is that the Bott element acts on the spectral sequence of Theorem 7.6 and this action has the expected behavior. The formal assertion is given by the following result. Recall that $b \in \mu_{n}(k) \cong \mathrm{H}_{\text {ét }}^{0}\left(\operatorname{Spec} k, \mu_{n}\right)$ denotes a chosen primitive $n$-th root of unity and $\beta \in K_{2}(k ; \mathbb{Z} / n) \cong \mathbb{Z} / n$ maps to $b$ under the boundary map $K_{2}(k ; \mathbb{Z} / n) \rightarrow K_{1}(k) \cong k^{\times}$.

Proposition 10.1. Let $k$ be an algebraically closed field and assume $n \geq 1$ is an integer relatively prime to the exponential characteristic of $k$ satisfying (Condition 2-3). For any Cohen-Macaulay, quasi-projective $k$-variety $U$, there is an endomorphism of bidegree $(0,2)$ of the exact couple $\left(D_{s, t}^{r}, E_{s, t}^{r}\right)$ defining the first spectral sequence

$$
E_{s, t}^{2}=\mathrm{H}_{c}^{s}\left(U, \mu_{n}^{\otimes t / 2}(k)\right) \Longrightarrow K_{s+t}^{B M}(U ; \mathbb{Z} / n)
$$

of Theorem 7.6 that is given by multiplication by $b$ on $E^{2}$ terms and that is given by multiplication by $\beta$ on the abutment.

If $U$ is smooth, this determines an endomorphism of bidegree $(-1,-1)$ of the exact couple $\left(D_{r}^{p, q}, E_{r}^{p, q}\right)$ defining the second spectral sequence

$$
E_{2}^{p, q}=\mathrm{H}_{e t}^{p-q}\left(U, \mu_{n}^{\otimes(-q)}(k)\right) \Longrightarrow K_{-p-q}^{B M}(U ; \mathbb{Z} / n)
$$

of Theorem 7.6 that is given by multiplication by $b$ on $E_{2}$ terms and that is given by multiplication by $\beta$ on the abutment.

These endomorphisms are natural with respect to quasi-finite, flat morphisms.

Proof. Recall that the first spectral sequence of Theorem 7.6 arises from a tower of spaces depending functorially on $U$,

$$
\cdots \rightarrow G_{t+1}(U) \rightarrow G_{t}(U) \rightarrow \cdots \rightarrow G_{0}(U) \rightarrow *,
$$

together with a family of fibration sequences of the form

$$
F_{t}(U) \rightarrow G_{t}(U) \rightarrow G_{t-1}(U), \quad t \geq 0 .
$$

Specifically, we have

$$
G_{t}(V)=\operatorname{diag}\left(d \mapsto P_{t} \mathcal{K}^{q f}\left(\Delta^{d}, V ; \mathbb{Z} / n\right)\right)
$$

and

$$
F_{t}(V)=\operatorname{diag}\left(d \mapsto Q_{t} \mathcal{K}^{q f}\left(\Delta^{d}, V ; \mathbb{Z} / n\right)\right),
$$

and the exact couple defining the spectral sequence is given by the formulas

$$
D_{s, t}^{2}=\pi_{s+t+1} G_{t-1}(U)
$$

and

$$
E_{s, t}^{2}=\pi_{s+t} F_{t}(U) \cong \mathrm{H}_{c}^{s}\left(U, \mu^{t / 2}(k)^{\#}\right)^{\#} .
$$

Recall additionally that, in this context, $\mathcal{K}^{q f}(-, U ; \mathbb{Z} / n)$ is defined to be a functor from $S c h / k$ to Kan complexes given at $V \in S c h / k$ by the formula

$$
\mathcal{K}^{q f}(V, U ; \mathbb{Z} / n)=\underline{\operatorname{Maps}}_{*}\left(M, \Omega^{-2} \mathcal{K}^{q f}(V, U)\right),
$$

where $\Omega^{-2} \mathcal{K}^{q f}(V, U)=\operatorname{Ex}\left(\operatorname{diag}\left(S \bullet S \bullet \mathcal{P}^{q f}(V, U)\right)\right)$ and $M$ is the mod- $n$ Moore space. We extend the definition of $\Omega^{-2} \mathcal{K}^{q f}(V, U)$ by setting

$$
\Omega^{-p} \mathcal{K}^{q f}(V, U)=\operatorname{Ex}\left(\operatorname{diag}\left(S_{\bullet}^{(p)} \mathcal{P}^{q f}(V, U)\right)\right), \quad p \geq 1,
$$


where $S^{(p)}$ denotes $p$ iterates of the $S$ construction of Waldhausen (so that $S^{(p)} \mathcal{P}$ is a $p$-fold multi-simplicial set). Observe that there are natural pairings

$$
\Omega^{-p} \mathcal{K}^{q f}(V, U) \wedge \Omega^{-q} \mathcal{K}^{q f}(V, U) \rightarrow \Omega^{-p-q} \mathcal{K}^{q f}(V, U)
$$

induced by a tensor product over $\mathcal{O}_{V}$.

The Bott element is an element of $K_{2}(k ; \mathbb{Z} / n)$ and is given as the $\pi_{0}$ class of an element $\beta$ of the space $\Omega^{2} \mathcal{K}^{q f}(k ; \mathbb{Z} / n)=\underline{\text { Maps }}_{*}\left(S^{2} \wedge M, \Omega^{-2} \mathcal{K}(k)\right)$. Pairing with $\beta$ defines a natural transformation

$$
\left.\underline{\operatorname{Maps}}_{*}\left(M, \Omega^{-2} \mathcal{K}^{q f}(-, U)\right) \rightarrow \underline{\operatorname{Maps}} * \underline{H}^{2} \wedge M \wedge M, \Omega^{-4} \mathcal{K}^{q f}(-, U)\right),
$$

which, provided $n \not \equiv 2(\bmod 4)$, can be composed with precomposition along a "comultiplication" rule $S^{2} \wedge M \rightarrow M \wedge M$ (cf. [8, C.3]) to give a natural transformation

$$
\underline{\operatorname{Maps}}_{*}\left(M, \Omega^{-2} \mathcal{K}^{q f}(-, U)\right) \rightarrow \underline{\operatorname{Maps}}_{*}\left(S^{4} \wedge M, \Omega^{-4} \mathcal{K}^{q f}(-, U)\right) .
$$

There is a natural weak equivalence

$$
\underline{\operatorname{Maps}}_{*}\left(S^{2} \wedge M, \Omega^{-2} \mathcal{K}^{q f}(V, U)\right) \stackrel{\sim}{\longrightarrow} \underline{\operatorname{Maps}}_{*}\left(S^{4} \wedge M, \Omega^{-4} \mathcal{K}^{q f}(V, U)\right),
$$

which is induced by the natural transformation $S^{2} \wedge S_{\bullet}^{(2)} \mathcal{P} \rightarrow S_{\bullet}^{(4)} \mathcal{P}$ associated to any exact category $\mathcal{P}$. Also, for any Kan complex $Y$, we have the identification

$$
\underline{\operatorname{Maps}}_{*}\left(S^{2}, P_{t+2}(Y)\right)=P_{t}\left(\underline{\operatorname{Maps}}_{*}\left(S^{2}, Y\right)\right) \text {. }
$$

Combining the above natural maps together yields the following diagram of natural transformations of functors taking values in Kan complexes:

$$
\begin{gathered}
P_{t} \mathcal{K}^{q f}(-, U ; \mathbb{Z} / n) \\
=\downarrow \\
P_{t} \underline{\operatorname{Maps}}_{*}\left(M, \Omega^{-2} \mathcal{K}^{q f}(-, U)\right) \\
\beta \wedge-\downarrow \\
P_{t} \underline{\operatorname{Maps}}_{*}\left(S^{4} \wedge M, \Omega^{-4} \mathcal{K}^{q f}(-, U)\right) \\
\sim \uparrow \\
P_{t} \underline{\operatorname{Maps}}_{*}\left(S^{2} \wedge M, \Omega^{-2} \mathcal{K}^{q f}(-, U)\right) \\
\cong \downarrow \\
\underline{\operatorname{Maps}}_{*}\left(S^{2}, P_{t+2} \underline{\operatorname{Maps}}_{*}\left(M, \Omega^{-2} \mathcal{K}^{q f}(-, U)\right)\right. \\
=\downarrow \\
\underline{\operatorname{Maps}}_{*}\left(S^{2}, P_{t+2} \mathcal{K}^{q f}(-, U ; \mathbb{Z} / n)\right) .
\end{gathered}
$$

Applying each of the functors in (34) to $\Delta^{d}, d \geq 0$, we obtain a diagram of bisimplicial sets relating

$$
d \mapsto P_{t} \mathcal{K}^{q f}\left(\Delta^{d}, U ; \mathbb{Z} / n\right)
$$

to

$$
d \mapsto \underline{\operatorname{Maps}}_{*}\left(S^{2}, P_{t+2} \mathcal{K}^{q f}\left(\Delta^{d}, U ; \mathbb{Z} / n\right)\right)
$$


via a natural "zig-zag" diagram. Finally, on the level of geometric realizations we have the natural map

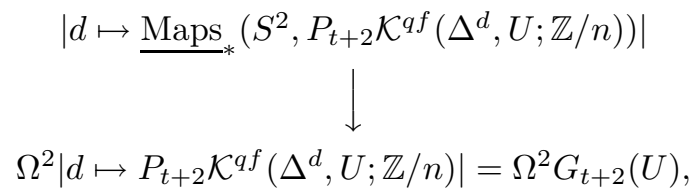

so that we have a natural zig-zag diagram relating $G_{t}(U)$ to $\Omega^{2} G_{t+2}(U)$. That is, there is a diagram of the form

$$
G_{t}(U) \longrightarrow \bullet \stackrel{\sim}{\longrightarrow} \longrightarrow \Omega^{2} G_{t+2}(U)
$$

in which the symbols $\bullet$ denote explicit functors in $U$ and the arrows are natural for quasi-finite, flat morphisms. Applying $\pi_{*}$ to this diagram for each $t \geq 0$ yields a natural map which we write as

$$
\beta \cap-: D_{*, *}^{2} \rightarrow D_{*, *+2}^{2} .
$$

The map $i: D_{s, t+1}^{2} \rightarrow D_{s+1, t}^{2}$ is induced by the natural transformation $P_{t} \rightarrow$ $P_{t-1}$, which clearly commutes with each of the natural transformations in (34). We therefore have a commutative diagram of the form

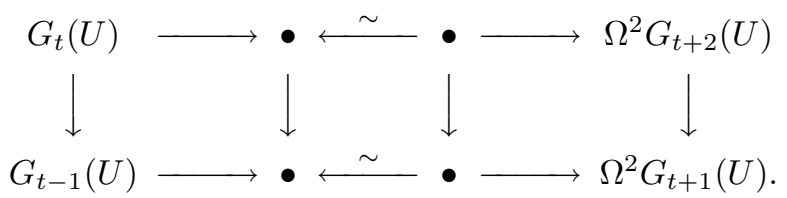

In particular, the square

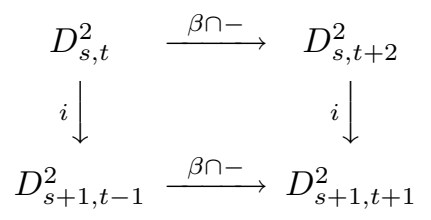

commutes, for all $s, t$. Moreover, when $t=1$ we have $D_{s-2,1}^{2}=K_{s}^{\mathrm{BM}}(U ; \mathbb{Z} / n)$, and it is apparent that the composition

$$
K_{s}^{\mathrm{BM}}(U ; \mathbb{Z} / n)=D_{s-2,1}^{2} \stackrel{\beta \cap-}{\longrightarrow} D_{s-2,3}^{2} \stackrel{i^{2}}{\longrightarrow} D_{s, 1}^{2}=K_{s+2}^{\mathrm{BM}}(U, \mathbb{Z} / n)
$$

coincides with $\beta \cap-$ as defined in Section 3 .

The exact same construction using $Q_{t}$ in place of $P_{t}$ produces a natural zig-zag map from $F_{t}(U)$ to $\Omega^{2} F_{t+2}(U)$ of the form

$$
F_{t}(U) \longrightarrow \bullet \longleftarrow \sim \Omega^{2} F_{t+2} .
$$

We write the induced map on homotopy groups $\pi_{s+t}$ as

$$
b \cap-: E_{s, t}^{2} \rightarrow E_{s, t+2}^{2},
$$


for all $s, t$. Moreover, we have a commutative diagram of the form

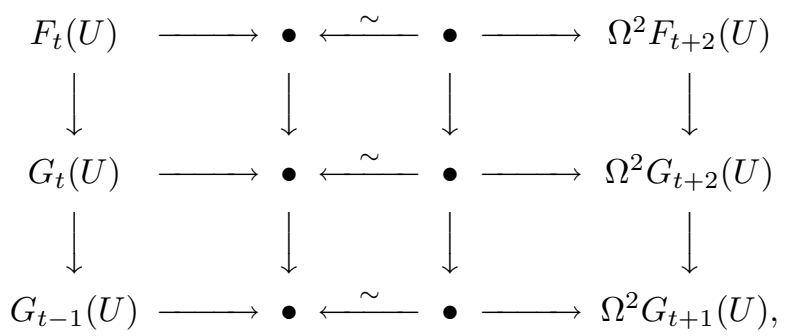

whose columns are fibration sequences and which is natural for quasi-finite, flat morphisms. This implies that the maps $i: D_{2}^{s, t} \rightarrow D_{2}^{s+1, t-1}, j: D_{2}^{s, t} \rightarrow E_{2}^{s, t}$, $k: E_{2}^{s, t} \rightarrow D_{2}^{s-2, t+1}$ determining the exact couple commute with the maps $\beta \cap-$ and $b \cap-$.

It remains to show that $b \cap-: E_{s, t}^{2} \rightarrow E_{s, t+2}^{2}$ coincides with multiplication by $b$ under the isomorphisms $\pi_{s+t} F_{t}(U) \cong \mathrm{H}_{c}^{s}\left(U, \mu_{n}^{\otimes(t / 2)}(k)^{\#}\right)^{\#}$ of Theorem 7.5. The map $b \cap-: E_{s, t}^{2} \rightarrow E_{s, t+2}^{2}$ is induced by the map of simplicial abelian groups

$$
\left(d \mapsto K_{t}^{q f}\left(\Delta^{d}, U ; \mathbb{Z} / n\right)\right) \stackrel{\beta \cap-}{\longrightarrow}\left(d \mapsto K_{t+2}^{q f}\left(\Delta^{d}, U ; \mathbb{Z} / n\right)\right)
$$

given by capping with $\beta \in K_{\text {geom }}^{2}(k ; \mathbb{Z} / n)$ degree-wise. Recall that the cap product determines a family of homotopy equivalences of simplicial abelian groups

$$
\left(d \mapsto K_{t}(k ; \mathbb{Z} / n) \otimes K_{0}^{q f}\left(\Delta^{d}, U\right)\right) \stackrel{\sim}{\longrightarrow}\left(d \mapsto K_{t}^{q f}\left(\Delta^{d}, U ; \mathbb{Z} / n\right)\right), \quad t \geq 0,
$$

by Theorem 7.1 (and Corollary 4.23). Under these homotopy equivalences, the map $b \cap-$ corresponds with the map

$$
\left(d \mapsto K_{t}(k ; \mathbb{Z} / n) \otimes K_{0}^{q f}\left(\Delta^{d}, U\right)\right) \stackrel{(\beta \cup-) \otimes i d}{\longrightarrow}\left(d \mapsto K_{t+2}(k ; \mathbb{Z} / n) \otimes K_{0}^{q f}\left(\Delta^{d}, U\right)\right)
$$

given by a degree-wise cup product of $\beta$ on $K_{*}(k ; \mathbb{Z} / n)$. By construction, $\beta$ maps to $b \in \mu_{n}(k) \cong{ }_{n} K_{1}(k)$ under the evident boundary map, which is an isomorphism. (The subscript $n$ denotes taking a subgroup of $n$-torsion elements.) Thus, under the isomorphisms

$$
\mu_{n}^{\otimes t / 2}(k) \cong\left({ }_{n} K_{1}(k)\right)^{\otimes t / 2} \cong K_{2}(k ; \mathbb{Z} / n)^{\otimes t / 2} \cong K_{t}(k ; \mathbb{Z} / n)
$$

(where the last map is defined by the cup product) and using the homotopy equivalence established in Theorem 7.5 we see that the map (35) corresponds to the map

$$
\left(d \mapsto \mu_{n}^{\otimes t / 2}(k) \otimes z_{\text {equi }}^{0}\left(\Delta^{d}, U\right)\right) \stackrel{(b \bullet-) \otimes i d}{\longrightarrow}\left(d \mapsto \mu_{n}^{\otimes(t+2) / 2}(k) \otimes z_{\text {equi }}^{0}\left(\Delta^{d}, U\right)\right) .
$$

Finally, for any finite $\mathbb{Z} / n$-module $F$, the isomorphism

$$
\pi_{p}\left(d \mapsto z_{\text {equi }}^{0}\left(\Delta^{d}, U\right) \otimes F\right) \cong \mathrm{H}_{c}^{p}\left(U, F^{\#}\right)^{\#}
$$

due to Suslin [26] (cf. Theorem 7.3) is natural in F. In particular, applying it to $\mu_{n}^{t / 2} \stackrel{b \bullet-}{\longrightarrow} \mu_{n}^{(t+2) / 2}$, we see that the map (35) corresponds to the map

$$
b \bullet-: \mathrm{H}_{c}^{s}\left(U, \mu_{n}^{\otimes(t / 2)}(k)^{\#}\right)^{\#} \rightarrow \mathrm{H}_{c}^{s}\left(U, \mu_{n}^{\otimes(t / 2)+1}(k)^{\#}\right)^{\#}
$$

under the given isomorphisms. 
We have thus established the existence of a natural (with respect to finite, flat morphisms) endomorphism of the exact couple $\left(D_{s, t}^{r}, E_{s, t}^{r}\right)$ having the asserted properties. The result for the other exact couple now follows by reindexing as in the proof of Theorem 7.6 using the fact that the Poincare duality isomorphism

$$
\mathrm{H}_{c}^{s}\left(U, F^{\#}\right)^{\#} \cong \mathrm{H}_{\text {ét }}^{2 d-s}\left(U, F \otimes_{\mathbb{Z} / n} \mu_{n}^{\otimes d}\right)
$$

is natural in $F$.

We now invert the action of $b$ and $\beta$ in the spectral sequence of Theorem 7.6 to obtain the main result of this paper.

Theorem 10.2. Let $U$ be a smooth, quasi-projective variety defined over an algebraically closed field $k$. Assume $n \geq 1$ is an integer relatively prime to the exponential characteristic of $k$ and such that $n$ satisfies (Condition 2-3). Then there is a convergent spectral sequence concentrated in the portion of the pq-plane satisfying $p \geq q \geq p-2 \operatorname{dim}(U)$ having the form

$$
E_{2}^{p, q}=\mathrm{H}_{\text {ét }}^{p-q}(U, \mathbb{Z} / n) \Longrightarrow K_{-p-q}(U ; \mathbb{Z} / n)[1 / \beta],
$$

where $K_{m}(U ; \mathbb{Z} / n)[1 / \beta]$ denotes the $m$-th graded piece of the result of inverting $\beta$ in the graded ring $K_{*}(U ; \mathbb{Z} / n)$. Moreover, this spectral sequence is natural with respect to quasi-finite, flat morphisms of smooth varieties.

Proof. Let $\left({ }^{\prime} D_{2}^{p, q},{ }^{\prime} E_{2}^{p, q}\right)$ denote the exact couple obtained by inverting the endomorphism $\beta \cap-$ constructed in Proposition 10.1 of the exact couple $\left(D_{2}^{p, q}, E_{2}^{p, q}\right)$ determining the the second spectral sequence of Theorem 7.6. (Formally, we have

$$
{ }^{\prime} D_{2}^{p, q}=\lim _{\longrightarrow}\left(D_{2}^{p, q} \stackrel{\beta \cap-}{\longrightarrow} D_{2}^{p-1, q-1} \stackrel{\beta \cap-}{\longrightarrow} \cdots \stackrel{\beta \cap-}{\longrightarrow} D_{2}^{p-m, q-m} \stackrel{\beta \cap-}{\longrightarrow} \cdots\right)
$$

and similarly for the ' $E_{2}$ terms.) Since $D_{2}^{p, q}$ vanishes for $p-q>2 d$ and multiplication by $\beta$ has degree $(-1,-1)$, we see that ${ }^{\prime} D_{2}^{p, q}=0$ for $p-q>2 d$ as well. Similarly, the map $i: D_{2}^{p+1, q-1} \rightarrow D_{2}^{p, q}$ is an isomorphism if $p-q<0$, and so $i:{ }^{\prime} D_{2}^{p+1, q-1} \rightarrow{ }^{\prime} D_{2}^{p, q}$ is also an isomorphism if $p-q<0$. Thus the spectral sequence associated to $\left({ }^{\prime} D_{2}^{p, q},{ }^{\prime} E_{2}^{p, q}\right)$ converges. The abutment of this spectral sequence is the graded abelian group

$$
\bigoplus_{m} \underset{\lim }{\longrightarrow}\left({ }^{\prime} D_{2}^{-m, 0} \stackrel{i}{\longrightarrow} D_{2}^{-m-1,1} \stackrel{i}{\longrightarrow} \cdots\right),
$$

which is given by the colimit of the $\mathbb{N} \times \mathbb{N}$ indexed diagram

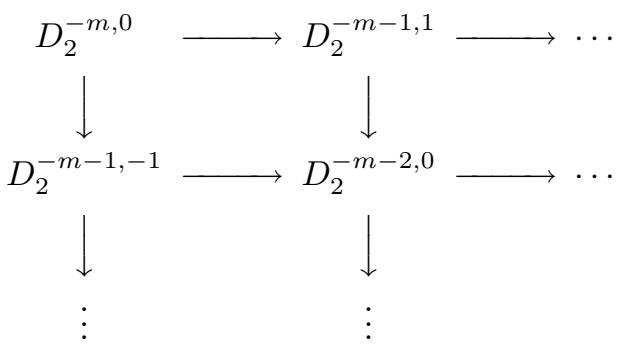

We can therefore identify the abutment with the graded abelian group

$$
\bigoplus_{m} \underset{\lim }{\longrightarrow}\left(D_{2}^{-m-d,-d} \rightarrow D_{2}^{-m-2-d,-d} \rightarrow \cdots\right)
$$


where the transition maps are given by composing $\beta \cap-$ with $i$. Since $D_{2}^{-j-d,-d}=$ $\pi_{j}\left|d \mapsto \mathcal{K}^{q f}\left(\Delta^{d}, U ; \mathbb{Z} / n\right)\right| \cong K_{j}^{\mathrm{BM}}(U ; \mathbb{Z} / n)$, for all $j$, we see that the abutment of the spectral sequence is the graded abelian group obtained by inverting the action of $\beta$ on $K_{*}^{\mathrm{BM}}(U ; \mathbb{Z} / n)$. By Theorems 8.13 and 9.3 , the abutment is isomorphic to $K_{*}(U ; \mathbb{Z} / n)[1 / \beta]$, and so we have a convergent spectral sequence

$$
{ }^{\prime} E_{2}^{p, q} \Longrightarrow K_{*}(U ; \mathbb{Z} / n)[1 / \beta] \text {. }
$$

Since $E_{2}^{p, q}=\mathrm{H}_{\text {ét }}^{p-q}\left(U, \mu_{n}^{\otimes-q}(k)\right)$ and the endomorphism we are inverting is multiplication by $b$, we have

$$
{ }^{\prime} E_{2}^{p, q}=\underline{\lim }\left(\mathrm{H}_{\text {ét }}^{p-q}\left(U, \mu_{n}^{\otimes-q}(k)\right) \stackrel{b \bullet-}{\longrightarrow} \mathrm{H}_{\text {ét }}^{p-q}\left(U, \mu_{n}^{\otimes-q+1}(k)\right) \stackrel{b \bullet-}{\longrightarrow} \cdots\right) .
$$

Since $b^{j} \bullet-: \mathbb{Z} / n \rightarrow \mu_{n}^{\otimes j}(k)$ is an isomorphism for all $j$, it follows that ${ }^{\prime} E_{2}^{p, q} \cong$ $\mathrm{H}_{\text {ét }}^{p-q}(U, \mathbb{Z} / n)$.

Remark 10.3. The spectral sequence of Theorem 10.2 is presumably natural with respect to arbitrary morphisms of smooth varieties. However, since it arises from the spectral sequence of Theorem 7.6, our techniques only imply the more limited naturality asserted above.

In fact, the results we have proven allow us to conclude a slightly stronger form of Thomason's theorem.

Theorem 10.4. Let $U$ be a smooth, quasi-projective variety of pure dimension $d$ defined over an algebraically closed field $k$. Assume $n \geq 1$ is an integer relatively prime to the exponential characteristic of $k$ and such that $n$ satisfies (Condition 2-3). Then the canonical map

$$
K_{*}^{B M}(U ; \mathbb{Z} / n) \rightarrow K_{*}^{B M}(U ; \mathbb{Z} / n)[1 / \beta]
$$

is an isomorphism in degrees $* \geq 2 d$ and thus the map

$$
\gamma_{U}: K_{*}^{B M}(U ; \mathbb{Z} / n) \rightarrow K_{\text {geom }}^{-*}(U ; \mathbb{Z} / n)
$$

is a split injection in degrees $* \geq 2 d$.

Consequently, the map

$$
K_{*}(U ; \mathbb{Z} / n) \rightarrow K_{*}(U ; \mathbb{Z} / n)[1 / \beta]
$$

is a split surjection in degrees $* \geq 2 d$, and in all degrees the kernel of this map is killed by $\beta^{d}$.

Proof. The proof of Theorem [10.2 establishes the existence of a map of spectral sequences from

$$
E_{2}^{p, q}=\mathrm{H}_{\text {ét }}^{p-q}\left(U, \mu_{n}^{\otimes(-q)}(k)\right) \Longrightarrow K_{-p-q}^{\mathrm{BM}}(U ; \mathbb{Z} / n)
$$

to

$$
{ }^{\prime} E_{2}^{p, q}=\mathrm{H}_{\text {ét }}^{p-q}(U, \mathbb{Z} / n) \Longrightarrow K_{-p-q}^{\mathrm{BM}}(U ; \mathbb{Z} / n)[1 / \beta]
$$

The $E_{2}$ terms vanish outside the portion of the $p q$-plane defined by $p \geq q \geq p-2 d$ and $q \leq-d$, whereas the ${ }^{\prime} E_{2}$ terms vanish outside the portion of the $p q$-plane defined by $p \geq q \geq p-2 d$. An easy induction argument shows that $E_{r}^{p, q}={ }^{\prime} E_{r}^{p, q}$ for all $r \geq 2$ such that for $p+q \leq-2 d$. It follows that $E_{\infty}^{p, q}={ }^{\prime} E_{\infty}^{p, q}$ and hence

$$
K_{-p-q}^{\mathrm{BM}}(U ; \mathbb{Z} / n) \cong K_{-p-q}^{\mathrm{BM}}(U ; \mathbb{Z} / n)[1 / \beta]
$$

whenever $-p-q \geq 2 d$. 
For any $i$, the composition of

$$
K_{2 d+i}^{\mathrm{BM}}(U ; \mathbb{Z} / n) \rightarrow K_{\text {geom }}^{-2 d-i}(U ; \mathbb{Z} / n) \rightarrow K_{4 d+i}^{\mathrm{BM}}(U ; \mathbb{Z} / n)
$$

is given by multiplication by $\beta^{d}$. When $i \geq 0$, this composition is isomorphic to multiplication by $\beta^{d}$ on $K_{2 d+i}^{\mathrm{BM}}(U ; \mathbb{Z} / n)[1 / \beta]$, and hence is an isomorphism. The asserted split injectivity therefore holds. Since

$$
K_{*}^{\mathrm{BM}}(U ; \mathbb{Z} / n)[1 / \beta] \cong K_{\text {geom }}^{-*}(U ; \mathbb{Z} / n)[1 / \beta]=K_{*}(U ; \mathbb{Z} / n)[1 / \beta],
$$

the asserted split surjectivity also follows. Similarly, for $i \geq 0$, the map

$$
\beta^{d}: K_{\text {geom }}^{-i}(U ; \mathbb{Z} / n) \rightarrow K_{\text {geom }}^{-i-2 d}(U ; \mathbb{Z} / n)
$$

factors through $K_{2 d+i}^{\mathrm{BM}}(U ; \mathbb{Z} / n) \cong K_{2 d+i}^{\mathrm{BM}}(U ; \mathbb{Z} / n)[1 / \beta]$ which implies $\operatorname{ker}\left(\beta^{d}\right)=$ $\operatorname{ker}\left(\beta^{d+1}\right)=\operatorname{ker}\left(\beta^{d+2}\right)=\cdots$, and thus

$$
\begin{aligned}
\operatorname{ker} & \left(K_{\text {geom }}^{-i}(U ; \mathbb{Z} / n) \rightarrow K_{\text {geom }}^{-i}(U ; \mathbb{Z} / n)[1 / \beta]\right) \\
& =\operatorname{ker}\left(K_{\text {geom }}^{-i}(U ; \mathbb{Z} / n) \stackrel{\beta^{d}}{\longrightarrow} K_{\text {geom }}^{-i-2 d}(U ; \mathbb{Z} / n)\right),
\end{aligned}
$$

for all $i \geq 0$.

We close with the following conjecture.

Conjecture 10.5. Let $U$ be a smooth, quasi-projective variety of pure dimension $d$ defined over an algebraically closed field $k$ and assume $n \geq 1$ is an integer relatively prime to the exponential characteristic of $k$. Then the map

$$
\gamma_{U}: K_{p}^{\mathrm{BM}}(U ; \mathbb{Z} / n) \rightarrow K_{\text {geom }}^{-p}(U ; \mathbb{Z} / n)
$$

is a surjection for $p \geq 2 d$ (and hence an isomorphism by Theorem 10.4).

In light of Theorem 10.4, Conjecture10.5 implies (a slightly weakened form of) the Quillen-Lichtenbaum conjecture. Namely, it implies that the natural map

$$
K_{*}(U ; \mathbb{Z} / n) \rightarrow K_{*}(U ; \mathbb{Z} / n)[1 / \beta]
$$

is an isomorphism in degrees $* \geq 2 \operatorname{dim}(U)$. (A modern way to express the strong form of the Quillen-Lichtenbaum conjecture is that this map is an isomorphism in degrees $* \geq \operatorname{dim}(U)-1$.)

\section{Appendix A. The Fulton-MacPherson axioms}

In this appendix, we give a complete proof of Theorem 3.10 showing that that the collection of graded abelian groups $K_{\text {geom }}^{*}(Y \rightarrow X)$ and $K_{g q f}^{*}(Y \rightarrow X)$ define "skew-commutative bivariant theories with units" in the sense of FultonMacPherson [10, 2.2]. Recall that part of the definition of a bivariant theory involves specifying classes of "independent squares" and "confined morphisms". For each of the two theories considered here, the class of independent squares consists of all pullback squares. For $K_{\text {geom }}^{*}$ every morphism is a confined morphism, while for $K_{g q f}^{*}$ the confined morphisms are the proper morphisms. For the convenience of the reader, we list the Fulton-MacPherson axioms in full detail below.

Throughout this appendix, we write

$$
\mathcal{P}(Y \rightarrow X), \quad \mathcal{K}(Y \rightarrow X), \quad \mathcal{K}_{\mathrm{g}}(Y \rightarrow X),
$$

for either of the two triples

$$
\mathcal{P}^{\text {fin }}(Y \rightarrow X), \quad \mathcal{K}^{\text {fin }}(Y \rightarrow X), \quad \mathcal{K}_{\text {geom }}(Y \rightarrow X),
$$


or

$$
\mathcal{P}^{q f}(Y \rightarrow X), \quad \mathcal{K}^{q f}(Y \rightarrow X), \quad \mathcal{K}_{g q f}(Y \rightarrow X) .
$$

Moreover, we let $K^{*}(Y \rightarrow X)$ denote either

$$
K_{\text {geom }}^{*}(Y \rightarrow X ; \mathbb{Z} / n) \quad \text { or } \quad K_{g q f}^{*}(Y \rightarrow X ; \mathbb{Z} / n),
$$

where $n$ is an integer (possibly 0) satisfying (Condition 2-3). The terms "independent square" and "confined morphism" will be used as indicated above.

The axioms required of the classes of independent squares and confined morphisms are given in [10, 2.1] and will not be recalled here, but the reader may readily verify that in each of the two cases above, these axioms are satisfied. The Fulton-MacPherson axioms of a bivariant theory assert that the pushforward maps (along confined morphisms), pullback maps (along arbitrary morphisms), and pairings (for arbitrary pairs of composable morphisms) given in (3) and (4) above satisfy the following conditions:

(A1) Associativity of Product: Given morphisms

$$
X \stackrel{f}{\longrightarrow} Y \stackrel{g}{\longrightarrow} Z \stackrel{h}{\longrightarrow} W
$$

and elements

$$
\alpha \in K^{p}(X \rightarrow Y), \quad \beta \in K^{q}(Y \rightarrow Z), \quad \text { and } \gamma \in K^{r}(Z \rightarrow W)
$$

we have

$$
(\alpha \bullet \beta) \bullet \gamma=\alpha \bullet(\beta \bullet \gamma) \in K^{p+q+r}(Z \rightarrow W) .
$$

(A2) Functoriality of Pushforward: Given morphisms

$$
X \stackrel{f}{\longrightarrow} Y \stackrel{g}{\longrightarrow} Z \stackrel{h}{\longrightarrow} W
$$

with $f$ and $g$ confined and an element $\alpha \in K^{p}(X \rightarrow W)$, we have

$$
(g f)_{*}(\alpha)=g_{*}\left(f_{*}(\alpha)\right) \in K^{p}(Z \rightarrow W) .
$$

(A3) Functoriality of Pullback: Given a commutative diagram

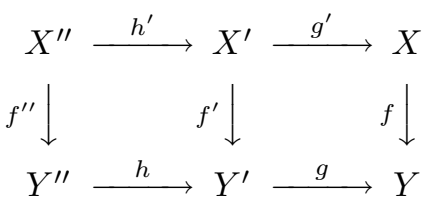

consisting of two independent squares and an element $\alpha \in K^{p}(X \rightarrow Y)$, we have

$$
(g h)^{*}(\alpha)=h^{*}\left(g^{*}(\alpha)\right) \in K^{p}\left(X^{\prime \prime} \rightarrow Y^{\prime \prime}\right) .
$$

$\left(\mathrm{A}_{12}\right)$ Commutativity of Pushforward with Product: Given morphisms

$$
X \stackrel{f}{\longrightarrow} Y \stackrel{g}{\longrightarrow} Z \stackrel{h}{\longrightarrow} W
$$

with $f$ confined and given $\alpha \in K^{p}(X \rightarrow Z)$ and $\beta \in K^{q}(Z \rightarrow W)$, we have

$$
f_{*}(\alpha \bullet \beta)=f_{*}(\alpha) \bullet \beta \in K^{p+q}(Y \rightarrow W) .
$$


$\left(\mathrm{A}_{13}\right)$ Commutativity of Pullback with Product: Given a commutative diagram

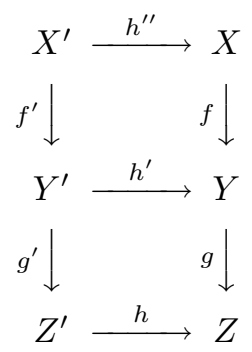

of independent squares and given $\alpha \in K^{p}(X \rightarrow Y)$ and $\beta \in K^{q}(Y \rightarrow Z)$, we have

$$
h^{*}(\alpha \bullet \beta)=\left(h^{\prime}\right)^{*}(\alpha) \bullet h^{*}(\beta) \in K^{p+q}\left(X^{\prime} \rightarrow Z^{\prime}\right) .
$$

$\left(\mathrm{A}_{23}\right)$ Commutativity of Pullback with Pushforward: Given a commutative diagram

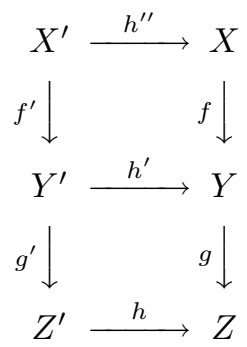

of independent squares with $f$ confined and given $\alpha \in K^{p}(X \rightarrow Z)$, we have

$$
f_{*}^{\prime}\left(h^{*}(\alpha)\right)=h^{*} f_{*}(\alpha) \in K^{p}\left(Y^{\prime} \rightarrow Z^{\prime}\right) .
$$

$\left(\mathrm{A}_{123}\right)$ Projection Formula: Given a commutative diagram

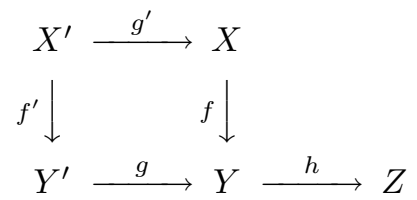

with an independent square and $g$ confined and given $\alpha \in K^{p}(X \rightarrow Y)$ and $\beta \in K^{q}\left(Y^{\prime} \rightarrow Z\right)$, we have

$$
g_{*}^{\prime}\left(g^{*}(\alpha) \bullet \beta\right)=\alpha \bullet g_{*}(\beta) \in K^{p+q}(X \rightarrow Z) .
$$

In addition, the bivariant theory $K^{*}$ has units and is skew-commutative provided the following axioms hold:

(Units) For any $X$ in $S c h / k$, there is an element $1_{X} \in K^{0}(X \rightarrow X)$ such that

$$
\alpha \bullet 1_{X}=\alpha \quad \text { and } \quad 1_{X} \bullet \beta=\beta
$$

for all $\alpha \in K^{*}(W \rightarrow X)$ and $\beta \in K^{*}(X \rightarrow Y)$ and such that

$$
g^{*}\left(1_{X}\right)=1_{X^{\prime}}
$$

for any morphism $g: X^{\prime} \rightarrow X$. 
(Skew-commutativity) For any independent square

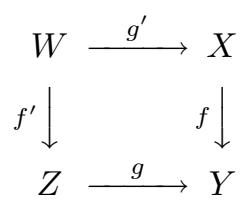

and any elements $\alpha \in K^{m}(X \rightarrow Z)$ and $\beta \in K^{n}(Y \rightarrow Z)$, we have

$$
g^{*}(\alpha) \bullet \beta=(-1)^{m n} f^{*}(\beta) \bullet \alpha .
$$

Proof of Theorem 3.10. Axiom (A1) follows immediately from Proposition 3.9 and the fact that the mod- $n$ Moore space admits a suitable coproduct rule for such values of $n$. Axioms (A2) and (A3) follow immediately from Propositions 3.6 and 3.8 .

To prove Axiom $\left(\mathrm{A}_{12}\right)$, we use the fact that the diagram

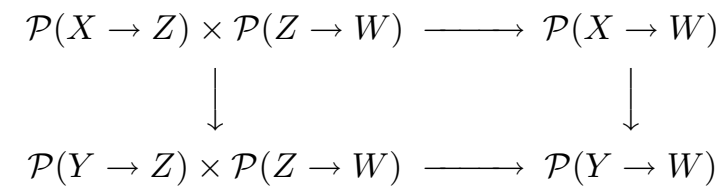

of exact functors commutes up to the canonical isomorphisms

$$
f_{*}\left(M \otimes_{\mathcal{O}_{X}}(g \circ f)^{*} N\right) \cong f_{*}\left(M \otimes_{\mathcal{O}_{X}} f^{*}\left(g^{*} N\right)\right) \cong f_{*} M \otimes_{\mathcal{O}_{Y}} g^{*} N
$$

and that each of the two possible compositions in this square yields a biexact functor. (Recall from Remark 3.2 and the proof of Proposition 3.9 that the categories and functors appearing in this square can be made strictly functorial with respect to pullback along $W$.) Thus, for each $d \geq 0$, there is a homotopy joining the two possible maps of the form

$$
\mathcal{K}\left(X \times \Delta^{d} \rightarrow Z \times \Delta^{d}\right) \wedge \mathcal{K}\left(Z \times \Delta^{d} \rightarrow W \times \Delta^{d}\right) \rightarrow \mathcal{K}\left(Y \times \Delta^{d} \rightarrow W \times \Delta^{d}\right),
$$

and the canonical nature of the isomorphisms inducing these homotopies implies that they are compatible with the simplicial structure. Hence, there is a homotopy relating the two induced maps of simplicial spaces of the form

$\left(d \mapsto \mathcal{K}\left(X \times \Delta^{d} \rightarrow Z \times \Delta^{d}\right) \wedge \mathcal{K}\left(Z \times \Delta^{d} \rightarrow W \times \Delta^{d}\right)\right) \rightarrow\left(d \mapsto \mathcal{K}\left(Y \times \Delta^{d} \rightarrow W \times \Delta^{d}\right)\right)$, from which Axiom $\left(\mathrm{A}_{12}\right)$ follows.

To establish Axiom $\left(\mathrm{A}_{13}\right)$, note that the square

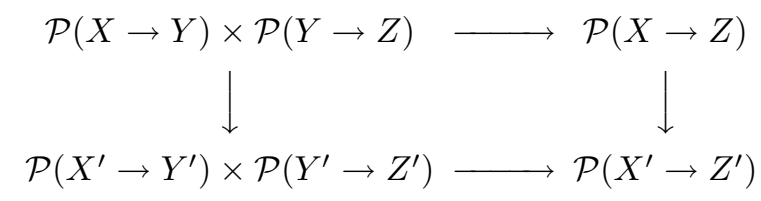

commutes up to the canonical isomorphisms

$$
\left(h^{\prime \prime}\right)^{*}\left(M \otimes_{\mathcal{O}_{X}} f^{*} N\right) \cong\left(h^{\prime \prime}\right)^{*}(M) \otimes_{\mathcal{O}_{X^{\prime}}}\left(h^{\prime \prime}\right)^{*} f^{*} N \cong\left(h^{\prime \prime}\right)^{*}(M) \otimes_{\mathcal{O}_{X^{\prime}}}\left(f^{\prime}\right)^{*}\left(h^{\prime}\right)^{*} N,
$$

and that each of the two possible compositions yields a biexact functor. As before, this leads to a homotopy joining the two possible maps of the form

$$
\mathcal{K}_{\mathrm{g}}(X \rightarrow Y) \wedge \mathcal{K}_{\mathrm{g}}(Y \rightarrow Z) \rightarrow \mathcal{K}_{\mathrm{g}}\left(X^{\prime} \rightarrow Z^{\prime}\right)
$$


To establish Axiom $\left(\mathrm{A}_{23}\right)$, note that

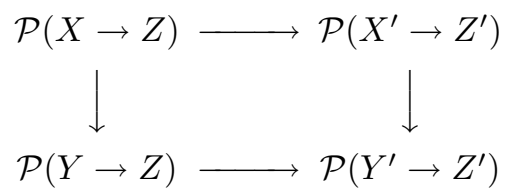

commutes up to the canonical isomorphism

$$
f_{*}^{\prime}\left(\left(h^{\prime \prime}\right)^{*} M\right) \cong h^{\prime *}\left(f_{*} M\right)
$$

which holds since the higher derived functors of $f_{*}$ and $f_{*}^{\prime}$ vanish and since the higher Tor sheaves $\mathcal{T}$ or $_{*}^{\mathcal{O}_{X}}\left(\mathcal{O}_{X^{\prime}},-\right)$ and $\mathcal{T}$ or $_{*}^{\mathcal{O}_{Y}}\left(\mathcal{O}_{Y^{\prime}},-\right)$ vanish for objects in $\mathcal{P}(X \rightarrow Y)$. (The vanishing of the higher derived functors of $f_{*}$ and $f_{*}^{\prime}$ holds since objects in $\mathcal{P}(X \rightarrow Z)$ (respectively, $\mathcal{P}\left(X^{\prime} \rightarrow Z^{\prime}\right)$ ) have support finite over $Y$ (respectively, $Y^{\prime}$ ). Note that in the case $\mathcal{P}=\mathcal{P}^{q f}$, the finiteness of supports holds since the supports are quasi-finite and $f$ and $f^{\prime}$ are assumed proper.) As before, such canonical isomorphisms establish the homotopy commutativity of

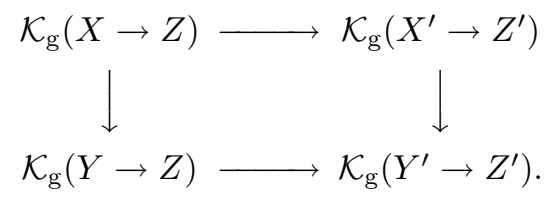

To establish Axiom $\left(\mathrm{A}_{123}\right)$, we use the fact that the diagram of functors

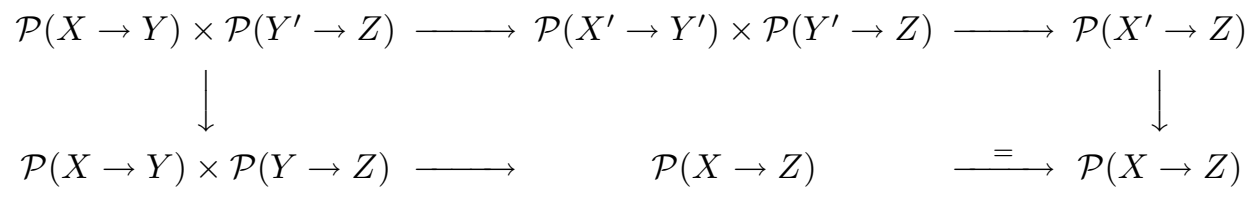

commutes up to a canonical isomorphism by using the chain of canonical isomorphisms

$$
\left(g^{\prime}\right)_{*}\left(\left(g^{\prime}\right)^{*} M \otimes_{\mathcal{O}_{X^{\prime}}}\left(f^{\prime}\right)^{*} N\right) \cong M \otimes_{\mathcal{O}_{X}} g_{*}^{\prime}\left(f^{\prime}\right)^{*} N \cong M \otimes_{\mathcal{O}_{X}} f^{*} g_{*}(N) .
$$

As before, this chain of isomorphisms exists since all relevant higher derived images and Tor modules vanish. This gives the homotopy commutativity of the associated diagram of spaces

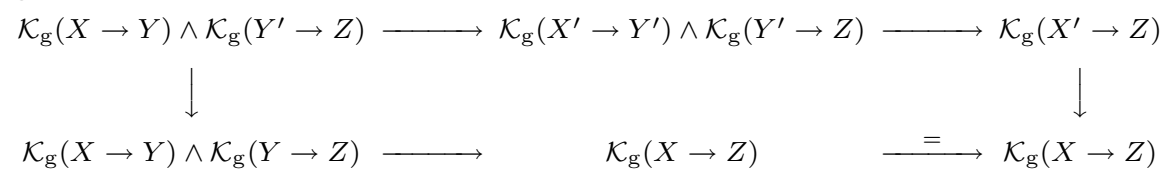

We have thus shown that both $\mathcal{K}_{\text {geom }}^{*}$ and $\mathcal{K}_{g q f}^{*}$ determine bivariant theories. The unit elements for each theory are given by the classes $\left[\mathcal{O}_{X}\right] \in K_{\mathrm{g}}^{0}(X \rightarrow X ; \mathbb{Z} / n) \cong$ $K_{\text {geom }}^{0}(X ; \mathbb{Z} / n)$, which are readily seen to satisfy the required properties. To establish skew-commutativity, note that the square

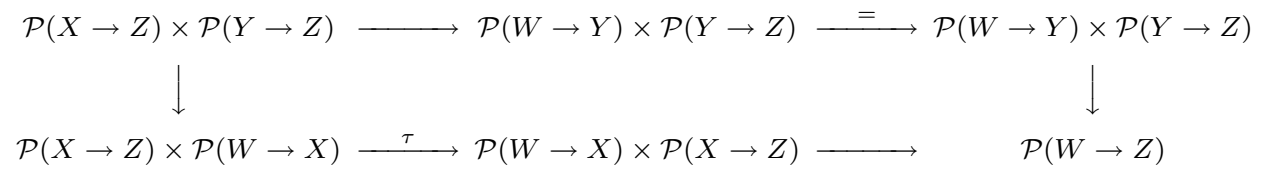

commutes up to the canonical isomorphism

$$
\left(g^{\prime}\right)^{*} M \otimes_{\mathcal{O}_{W}}\left(f^{\prime}\right)^{*} N \cong\left(f^{\prime}\right)^{*} N \otimes_{\mathcal{O}_{W}}\left(g^{\prime}\right)^{*}(M),
$$


where $\tau$ is the evident transposition. As before, this gives the homotopy commutative diagram of spaces

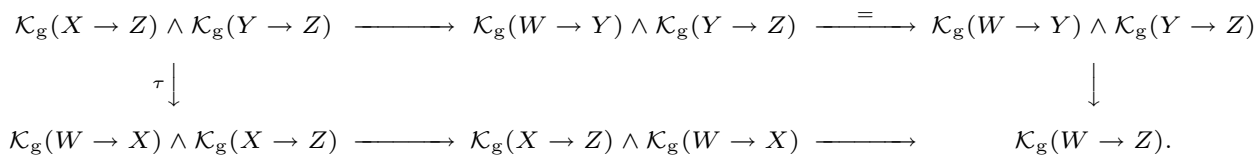

The desired formula follows. (On the level of homotopy classes represented by maps $\alpha: S^{m} \rightarrow \mathcal{K}_{\mathrm{g}}(X \rightarrow Z)$ and $\beta: S^{n} \rightarrow \mathcal{K}_{\mathrm{g}}(Y \rightarrow Z)$, the map $\tau$ introduces a sign of $(-1)^{m n}$ since the endomorphism of $S^{m+n}$ given as the composition of

$$
S^{m+n} \cong S^{m} \wedge S^{n} \cong S^{n} \wedge S^{m} \cong S^{n+m}
$$

has degree $(-1)^{m n}$.)

The inclusion of $\mathcal{P}^{\text {fin }}(X \rightarrow Y)$ into $\mathcal{P}^{q f}(X \rightarrow Y)$ is clearly compatible with the pushforward, pullback, and product operations defined in Propositions 2.3 and 2.4 and thus the collection of maps

$$
K_{\text {geom }}^{*}(X \rightarrow Y) \rightarrow K_{g q f}^{*}(X \rightarrow Y)
$$

determines a Grothendieck transformation of bivariant theories (cf. [10, 2.7.1]).

\section{REFERENCES}

[1] M. Artin, A. Grothendieck, and J. L. Verdier. SGA4: Théries des Topos et Cohomologie Étale des Schémas, Tome 3, volume 305 of Lecture Notes in Math. Springer-Verlag, Berlin, Heidelberg, New York, 1973. MR 50:7132

[2] Hyman Bass. Algebraic K-theory. W. A. Benjamin, New York, Amsterdam, 1968. MR 40:2736

[3] Spencer Bloch and Steven Lichtenbaum. A spectral sequence for motivic cohomology. Preprint. Available at http://www.math.uiuc.edu/K-theory/0062/.

[4] A. K. Bousfield. On the homology spectral sequence of a cosimplicial space. Amer. J. Math., 109(2):361-394, 1987. MR 88j:55017

[5] A. K. Bousfield and E. M. Friedlander. Homotopy of $\Gamma$-spaces, spectra and bisimplicial sets. In M. G. Barret and M. E. Mahowald, editors, Geometric Applications of Homotopy Theory II, volume 658, pages 80-130. Springer-Verlag, 1978. MR 80e:55021

[6] A. K. Bousfield and D. M. Kan. Homotopy Limits, Completions and Localizations, volume 304 of Lecture Notes in Math. Springer-Verlag, 1972. MR 51:1825

[7] A. J. de Jong. Smoothness, semi-stability and alterations. Inst. Hautes Études Sci. Publ. Math., (83):51-93, 1996. MR 98e:14011

[8] Eric M. Friedlander and Andrei Suslin. The spectral sequence relating algebraic $K$-theory to motivic cohomology. Ann. Ec. Norm. Sup., 35:773-875, 2002.

[9] Eric M. Friedlander and Vladimir Voevodsky. Bivariant cycle cohomology. In Higher Chow groups and étale cohomology, pages 138-187. Princeton Univ. Press, Princeton, NJ, 2000. MR 2001d:14026

[10] William Fulton and Robert MacPherson. Categorical framework for the study of singular spaces. Mem. Amer. Math. Soc., 31(243):vi+165, 1981. MR 83a:55015

[11] Daniel R. Grayson. Higher algebraic K-theory: II [after D. Quillen]. In Algebraic K-theory, volume 551 of Lecture Notes in Math. Springer-Verlag, Berlin, Heidelberg, New York, 1976. MR 58:28137

[12] Daniel R. Grayson. Projections, cycles, and algebraic K-theory. Math. Ann., 234:69-72, 1978. MR 58:10891

[13] Daniel R. Grayson. Weight filtrations in algebraic $K$-theory. In Motives, volume 55 of Proceedings of Symposia in Pure Mathematics, pages 207-244. American Mathematical Society, 1994. MR 95a:19006

[14] Daniel R. Grayson. Weight filtrations via commuting automorphisms. K-theory, 9:139-172, 1995. MR 96h:19001 
[15] Daniel R. Grayson and Mark E. Walker. Geometric models for algebraic $K$-theory. $K$-theory, 20(4):311-330, 2000. MR 2001m:19006

[16] Robin Hartshorne. Algebraic Geometry. Springer-Verlag, Berlin, Heibelberg, New York, 1977. MR 57:3116

[17] Marc Levine. $K$-theory and motivic cohomology of schemes. Preprint. Available at http: //www.math.uiuc.edu/K-theory/0336/, February 1999.

[18] Hideyuki Matsumura. Commutative Ring Theory. Cambridge University Press, Cambridge, 1990. MR 90i:13001

[19] J. S. Milne. Étale Cohomology. Princeton University Press, Princeton, New Jersey, 1980. MR 81j:14002

[20] Daniel Quillen. Higher algebraic K-theory: I. In Algebraic K-theory I, volume 341 of Lecture Notes in Math. Springer-Verlag, Berlin, Heidelberg, New York, 1972. MR 49:2895

[21] Michel Raynaud and Laurent Gruson. Critères de platitude et de projectivité. Techniques de "platification" d'un module. Invent. Math., 13:1-89, 1971. MR 46:7219

[22] J. P. Serre. Algébra Locale, Multiplicités, volume 11 of Lecture Notes in Math. SpringerVerlag, Berlin, Heidelberg, New York, 1965. MR 34:1352

[23] A. Suslin. On the K-theory of local fields. J. Pure. and Appl. Algebra, 34:301-318, 1984. MR 86d:18010

[24] A. Suslin and V. Voevodsky. Singular homology of abstract algebraic varieties. Invent. Math., 123:61-94, 1996. MR 97e:14030

[25] Andrei Suslin and Vladimir Voevodsky. Relative cycles and Chow sheaves. In Cycles, transfers, and motivic homology theories, pages 10-86. Princeton Univ. Press, Princeton, NJ, 2000. MR 2001d:14026

[26] Andrei A. Suslin. Higher Chow groups and étale cohomology. In Cycles, transfers, and motivic homology theories, pages 239-254. Princeton Univ. Press, Princeton, NJ, 2000. MR 2001d:14026

[27] R. Thomason. Algebraic K-theory and étale cohomology. Ann. Sceint. Éc. Norm. Sup., 18:437-552, 1985. MR 87k:14016

[28] R. W. Thomason and T. Trobaugh. Higher algebraic $K$-theory of schemes and of derived categories. In The Grothendieck Festschrift, Volume III, volume 88 of Progress in Math., pages 247-436. Birkhäuser, Boston, Basél, Berlin, 1990. MR 92f:19001

[29] Vladimir Voevodsky. Cohomological theory of presheaves with transfers. In Higher Chow groups and étale cohomology, pages 87-137. Princeton Univ. Press, Princeton, NJ, 2000. MR 2001d:14026

[30] Friedhelm Waldhausen. Algebraic K-theory of generalized free products. I, II. Ann. of Math. (2), 108(1):135-204, 1978. MR 58:16845a

[31] Friedhelm Waldhausen. Algebraic $K$-theory of spaces. In Algebraic and geometric topology (New Brunswick, N.J., 1983), pages 318-419. Springer, Berlin, 1985. MR 86m:18011

[32] Mark E. Walker. Motivic Complexes and the K-theory of Automorphisms. Ph.D. thesis, University of Illinois, Urbana-Champaign, 1996. Available at http://www.math.unl.edu/ $\sim$ mwalker.

[33] Mark E. Walker. The primitive topology of a scheme. J. Algebra, 201(2):656-685, 1998. MR 99b:14019

[34] Mark E. Walker. Adams operations for bivariant $K$-theory and a filtration using projective lines. K-theory, 21(2):101-140, 2000. MR 2002i:19005

[35] Mark E. Walker. Weight zero motivic cohomology and the general linear group of a simplicial ring. J. Pure Appl. Algebra, 147(3):311-319, 2000. MR 2001b:19003

[36] Mark E. Walker. Semi-topological $K$-homology and Thomason's theorem. K-Theory, 26(3):207-286, 2002. MR 2003k:19004

[37] C. Weibel. Homotopy algebraic K-theory. In Algebraic K-theory and Number Theory, volume 83 of Contemporary Mathematics, pages 461-488. American Mathematical Society, 1989. MR 90d:18006

[38] Charles A. Weibel. Negative $K$-theory of varieties with isolated singularities. In Proceedings of the Luminy conference on algebraic K-theory (Luminy, 1983), volume 34, pages 331-342, 1984. MR 86d:14015

Department of Mathematics, University of Nebraska, Lincoln, Nebraska 68588-0323

E-mail address: mwalker@math.unl.edu 